Pacific

Journal of

Mathematics

STABLE FLAGS, TRIVIALIZATIONS

AND REGULAR CONNECTIONS

Elie COMPOINT AND EduARDo COREL

Volume $263 \quad$ No. 2

June 2013 


\title{
STABLE FLAGS, TRIVIALIZATIONS AND REGULAR CONNECTIONS
}

\author{
Elie COMPOINT AND EduARdo COREL
}

\begin{abstract}
We study stalkwise modifications of a holomorphic vector bundle endowed with a meromorphic connection on a compact Riemann surface. We introduce the notion of Birkhoff-Grothendieck trivialization, in the case of the Riemann sphere, and show that its computation corresponds to shortest paths in some local affine Bruhat-Tits building. We use this to compute how the type of a bundle changes under stalk modifications, and give several corresponding algorithmic procedures. We finally deduce from these results some applications to the Riemann-Hilbert problem.
\end{abstract}

\section{Introduction}

The motivation of this article originates in the Riemann-Hilbert problem on a compact Riemann surface, and the present work follows it as a guideline. The results presented herein are however not directly related to this problem. The reader who is exclusively interested in new advances on the Riemann-Hilbert problem will nevertheless find a couple of improvements on already known results. The real interest of the present paper in the eyes of its authors consists in translating this classical problem in a "new" setting (the setting of Bruhat-Tits buildings). In this new context, the Riemann-Hilbert problem reveals new geometric objects (such as Birkhoff-Grothendieck trivializations), whose study appears to be interesting by itself, and seems also promising for the original problem.

The Riemann-Hilbert problem (RHP) has a long and distinguished history, not even devoid of suspense, for it has been solved several times, using different tools, in a seemingly complete and positive way. It was finally A. A. Bolibrukh, in a celebrated series of papers at the beginning of the 1990s, who clarified the situation, by rigorously defining (and exhibiting a counterexample to) the strongest version of the RHP, thereby showing that people before him had either committed a mistake, or solved in reality a weaker problem.

MSC2010: primary 51N30, 34M03; secondary 34M50.

Keywords: meromorphic connection, vector bundle, Birkhoff-Grothendieck theorem, Bruhat-Tits building, Riemann-Hilbert problem. 
The modern approach to the RHP was initiated by H. Röhrl in the 1950s, who used the theory of vector bundles in a way that has been conserved since. First, one constructs a vector bundle $\mathscr{E}$ outside the singular points, whose cocycle mimics the monodromy. We call this the topological RH problem, since the monodromy is so much encoded in the topology of the constructed bundle, that construction of the required connection becomes essentially trivial. The second step consists of extending the bundle (and the connection) to the singular points by means of a local solution to the inverse monodromy problem. It has been exposed in great generality in [Deligne 1970] how to extend a holomorphic vector bundle $\mathscr{E}$, defined over the complement of a divisor $\mathscr{D}$ and endowed with a holomorphic connection $\nabla$ having a prescribed monodromy about $\mathscr{D}$, into a logarithmic connection $(\overline{\mathscr{E}}, \bar{\nabla})$ with singularities on the divisor.

In this way, we get all logarithmic extensions of $\mathscr{E}$ with nonresonant residue (the Deligne lattices). These two steps are sufficient to solve positively the weak Riemann-Hilbert problem (i.e., with regular singularities). Note, however, that in this second level, two different types of problems have been mixed. The connection constructed is essentially unique up to meromorphic equivalence whereas the holomorphic vector bundle setting already introduces much finer holomorphic equivalence problems. This fact can contribute to explain some of the confusions that have surrounded the precise formulation of the RHP.

The strong Riemann-Hilbert problem asks for a logarithmic bundle (with the prescribed monodromy) which is moreover trivial. So, to solve the Riemann-Hilbert problem in this way, one must modify the constructed Deligne bundle, over the support of the singular divisor exclusively (to keep the singular set invariant), while conserving its logarithmic character, until a trivial bundle is eventually found. Until Bolibrukh's celebrated counterexample [1990], it was widely acknowledged that this was possible, and it is indeed so in several "generic" instances, although some mistakes in the seemingly general solution by Plemelj had already been pointed out (e.g., in [Treibich Kohn 1983]).

The counterexample found by Bolibrukh to the strong Riemann-Hilbert problem requires the knowledge of all the logarithmic extensions of a regular connection, in order to prove that none is trivial. Despite the production of both counterexamples and sufficient conditions for a positive answer, no general necessary and sufficient conditions for the solubility of the strong Riemann-Hilbert problem have been given in terms of the monodromy representation only, except for the remarkable case of an irreducible representation [Bolibrukh 1990; Kostov 1992].

As already stated, the strong Riemann-Hilbert problem admits a solution if and only if the stalks of the Deligne bundle over the singular set can be replaced by logarithmic lattices in such a way that the resulting bundle is trivial. To tackle this problem, one should be able to 
(a) determine the set of all logarithmic lattices above a given point, and

(b) get a criterion for the triviality of the modified bundle.

Problem (a) can be considered classical, in the sense that it has been repeatedly solved, under different guises, going back as far as [Gantmacher 1959], and including [Levelt 1961; Babbitt and Varadarajan 1983; Bolibrukh 1990; Sabbah 2002]. In this paper, we give a complete description of the logarithmic lattices in terms of flags stabilized under the action of the residue of the connection; in a way, our contribution is to make the solution to problem (a) given in [Sabbah 2002] completely explicit and effective. We also give a partial answer to problem (b). In the case of $\mathbb{P}^{1}(\mathbb{C})$, the type of a vector bundle gives such a triviality criterion. In our approach, starting with the Deligne bundle $\mathfrak{D}$, we perform a modification of a finite number of stalks, resulting in the bundle $\mathfrak{D}^{\text {mod }}$. The question is then to compute the type of the modified bundle $\mathfrak{D}^{\text {mod }}$. Generalizing a result by Gabber and Sabbah (Proposition 28), we show how to determine the type of $\mathfrak{D}^{\text {mod }}$ from the type of $\mathfrak{D}$. Thus, problem (b) is reduced to computing the type of the Deligne bundle. In a second step, we show that this problem in turn is reduced to the well-known problem of connection matrices.

With these problems in mind, we introduce and study in this paper the notion of Birkhoff-Grothendieck trivialization of a bundle $\mathscr{E}$, which is a pointwise modification of $\mathscr{E}$ such that

(i) the resulting bundle $\mathscr{F}$ is holomorphically trivial, and

(ii) the relative elementary divisors of the stalks give the type of the bundle $\mathscr{E}$.

The paper is organized as follows. In a first section, we define the category in which we will work, and what we precisely mean by "modifying a bundle over one or several points". In a second part, we describe the geometry on the local lattices involved. We describe this geometry in terms of the affine Bruhat-Tits building of SL ${ }_{n}$.

The third part contains the main results of the paper. We use the previously introduced setting to give an effective method to compute how the type of an arbitrary bundle $E$ is modified under certain pointwise modifications. This algorithm can also be applied to compute the type of the bundle $E$. This third section concludes with a generalization of an essential result originally due to Bolibrukh, the permutation lemma, for which we provide an interesting geometric interpretation. This result allows us to give a quite complete insight into the structure of Birkhoff-Grothendieck trivializations, which we sum up as follows.

Theorem 1. Let $\Lambda^{0}$ be the set of pointwise modifications over $x \in \mathbb{P}^{1}(\mathbb{C})$ of a bundle $\mathscr{E}$ that give a trivial bundle. Then $M \in \Lambda^{0}$ is a Birkhoff-Grothendieck trivialization of $\mathscr{E}$ if and only if $M$ realizes the minimum of the canonical metric between $\mathscr{E}_{x}$ and $\boldsymbol{\Lambda}^{0}$ in the local affine Bruhat-Tits building of $\mathrm{SL}_{n}$ at $x$. 
The fourth section gives the complete description of the set of logarithmic lattices in terms of flags which are stable under the action of the residue of the connection on the Deligne bundle ${ }^{1}$.

In the last part, we use these tools for study of the Riemann-Hilbert problem. After recalling the construction of the classical Röhrl-Deligne bundle, we give a very short proof of Plemelj's theorem on the Riemann-Hilbert solubility. This wellknown result becomes an immediate consequence of the geometrical interpretations of the permutation lemma and the set of logarithmic lattices. We describe all trivializations of the Deligne bundle over an arbitrary point, and we give a concise proof of the Bolibrukh-Kostov theorem on the solvability of Riemann-Hilbert in the irreducible case. Finally, we give algorithmically effective procedures that allow to search the space of weak solutions.

\section{Holomorphic vector bundles}

Let $X$ be a compact Riemann surface and let $\pi: E \rightarrow X$ be a holomorphic vector bundle of rank $n$. The sheaf $\mathscr{E}$ of holomorphic sections of $E$ is a locally free sheaf of $\mathrm{O}_{X}$-modules of the same rank $n$, where $\mathrm{O}_{X}$ denotes as usual the sheaf of holomorphic functions on $X$. There is a well-known equivalence between these two categories. However, this equivalence does not hold for subobjects of the same rank. Therefore we will privilege the sheaf-theoretical formulation.

Meromorphic connections. Let $\mathscr{D}=\sum_{i=1}^{p} m_{i} x_{i}$ be a positive divisor on $X$. Let $\mathscr{O}_{\mathscr{D}}$ be the sheaf of meromorphic functions on $X$ having pole orders bounded by $\mathscr{D}$, and $|\mathscr{D}|=\left\{x_{1}, \ldots, x_{p}\right\}$ be the support of $\mathscr{D}$. For any finite set $S=\left\{y_{1}, \ldots, y_{t}\right\}$, let $(S)=y_{1}+\cdots+y_{t}$.

Let $\nabla: \mathscr{E} \rightarrow \mathscr{E} \otimes_{\mathscr{O}_{X}} \Omega_{X}^{1}(\mathscr{D})$ be a meromorphic connection with singular divisor $\mathscr{D}$ on a vector bundle $\mathscr{E}$ of rank $n$. Sometimes for simplicity we'll just say "connection" for the pair $(\mathscr{E}, \nabla)$. If we assume that $\mathscr{D}$ is the smallest possible (as we will do), then the Poincaré rank of $\nabla$ at $x \in X$ is the integer $\mathfrak{p}_{x}(\nabla)=\max \left(0, m_{x}-1\right)$. We will omit $\nabla$ whenever possible. If $\mathfrak{p}_{x}=0$, the sheaf $\mathscr{E}$ is said to be logarithmic at $x$. Let $\mathscr{S}=|\mathscr{D}|$ be the singular, and $\mathscr{S}_{\log }=\left\{x \in \mathscr{S} \mid \mathfrak{p}_{x}=0\right\}$ the logarithmic singular sets of $\nabla$. If $\mathscr{S}_{\log } \neq \varnothing$, then one can define the residue map $\operatorname{Res} \nabla \in \operatorname{End}\left(\mathscr{E} / \mathscr{E}_{-}\left(\mathscr{S}_{\text {log }}\right)\right.$ ). We will specify in parentheses the bundle if necessary.

The meromorphic bundle. Let $M_{X}$ be the sheaf of meromorphic functions on $X$ and let $\mathscr{V}=\mathscr{E} \otimes \mathbb{O}_{X}, M_{X}$ be the sheaf of meromorphic sections of $E$. A meromorphic connection $\nabla$ on $\mathscr{E}$ has a canonical extension to $\mathscr{V}$. Since the sheaf $\mathscr{E}$ can be

\footnotetext{
${ }^{1}$ Independently, P. Boalch [2011] has taken a similar view on local logarithmic lattices, in terms of stable filtrations and Bruhat-Tits buildings, albeit on the more general setting of a complex reductive group. Restricting to $\mathrm{GL}_{n}$ and $\mathrm{SL}_{n}$ enables us to give however more explicit formulæ; see Sections 3.2 and 3.3.
} 
embedded into $\mathscr{V}$, we consider from now on the set

$$
\mathscr{H}=\left\{\mathscr{F} \subset \mathscr{V} \mid \mathscr{F}_{\left.\stackrel{\text { loc. }}{\simeq} \mathcal{O}_{X}^{n}\right\}}\right\}
$$

of holomorphic vector bundles of $\mathscr{V}$. Each such bundle $\mathscr{F} \in \mathscr{H}$ is automatically endowed with a meromorphic connection induced by $\nabla$. For simplicity, we won't make any notational difference between all these connections.

We say that $\mathscr{F} \in \mathscr{H}$ is trivial if $\mathscr{F} \simeq 0_{X}^{n}$, or, equivalently, if $\mathscr{F}$ is generated by its global sections, and quasitrivial if there exists a line bundle $\mathscr{L}$ such that $\mathscr{F}_{\mathbb{O}} \otimes_{\mathcal{O}_{X}} \mathscr{L}$ is trivial. Let $\mathscr{H}_{0} \subset \mathscr{H}$ be the subset of trivial holomorphic bundles in $\mathscr{V}$. The following result is well known (e.g., [Sabbah 2002, p. 134]).

Lemma 2. Let $\mathscr{F}_{F} \in \mathscr{H}_{0}$ be a trivial holomorphic vector bundle in $(\mathscr{V}, \nabla)$. The space $\mathscr{F}(X)=\Gamma(X, \mathscr{F})$ of global sections is a $\mathbb{C}$-vector space of dimension $n$. For any logarithmic singularity $s \in \mathscr{Y}_{\log }(\mathscr{F})$, the residue $\operatorname{Res}_{s}^{\mathscr{F}} \nabla$ induces a well-defined endomorphism $\psi_{s} \in \operatorname{End}_{\mathbb{C}}(\mathscr{F}(X))$.

Stalks and lattices. For $\mathscr{F}_{F} \in \mathscr{H}$, the stalk $\mathscr{F}_{x}$ at any $x \in X$ is a free $\left(O_{X}\right)_{x}$-submodule of rank $n$ (or lattice) of $\mathscr{V}_{x}$, which is a vector space of dimension $n$ over $\left(M_{X}\right)_{x}$. Let $O_{x}=\widehat{\left(O_{X}\right)_{x}}$ be the formal completion of $\left(O_{X}\right)_{x}$, and $K_{x}=\operatorname{Frac}\left(O_{x}\right)$ its field

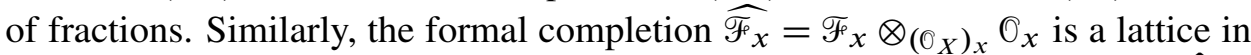
the vector space $V_{x}=\mathscr{V}_{x} \otimes_{\left(\mu_{X}\right)_{x}} K_{x}$. This operation is harmless, as $\Lambda \mapsto \widehat{\Lambda}$ is a bijection between the sets of lattices in $\mathscr{V}_{x}$ and $V_{x}$ (cf. [Malgrange 1996]). We define an equivalence relation $\sim_{x}$ on $\mathscr{H}$ as

$$
\mathscr{F}_{x} \sim_{x} \quad \text { if and only if } \mathscr{F}_{\mid} \mid X \backslash\{x\}=\widetilde{\mathscr{F}}_{\mid X \backslash\{x\}}
$$

For simplicity, we will drop the index $x$ as soon as no ambiguity can arise. Let $\boldsymbol{\Lambda}_{x}$ be the set of lattices in $V_{x}$. Any coset [F्F] of $\mathscr{H} / \sim_{x}$ can be identified with the set $\boldsymbol{\Lambda}_{x}$, by identifying $\mathscr{F}^{\prime} \in[\mathscr{F}]$ with its formal stalk $\widehat{\mathscr{F}}_{x}^{\prime} \in \boldsymbol{\Lambda}_{x}$ at $x$. Since $X$ is compact, two vector bundles $\mathscr{E}, \mathscr{F} \in \mathscr{H}$ have equal stalks outside a finite set.

Lemma 3. Let $\mathscr{E} \in \mathscr{H}$ be a holomorphic vector bundle. For any family of formal lattices $M_{x} \in \boldsymbol{\Lambda}_{x}$ for $x$ in a discrete set $\mathscr{Y}$, there exists a unique vector bundle $\mathscr{E}^{M} \in \mathcal{H}$ such that

$$
\left(\mathscr{C}^{M}\right)_{x}=\left\{\begin{array}{cl}
\mathscr{E}_{x} & \text { if } x \notin \mathscr{Y} \\
M_{x} \cap \mathscr{V}_{x} & \text { if } x \in \mathscr{S}
\end{array}\right.
$$

Conversely, for any $\mathscr{F}_{\mathscr{F}} \in \mathscr{H}$, there exists a discrete set $\mathscr{Y}$ and a family $\left(M_{x} \in \boldsymbol{\Lambda}_{x}\right)_{x \in \mathscr{S}}$ of lattices such that $\mathscr{F}=\mathscr{E} M$. If $\mathscr{E}$ is endowed with a meromorphic connection $\nabla$, there is a canonical extension $\nabla^{M}$ of $\nabla_{\mid X \backslash \varphi}$ as a meromorphic connection on $\mathscr{E} M$. In particular, a bundle $\mathscr{E} \in \mathscr{H}$ is completely determined by its coset $[\mathscr{E}] \in \mathscr{H} / \sim_{x}$ and the lattice $\widehat{\mathscr{E}_{x}} \in \boldsymbol{\Lambda}_{x}$. 
The sheaf $\mathscr{V}$ is trivial (as a meromorphic bundle), and the group $G$ of (meromorphic) automorphisms of the space $\Gamma(X, \mathscr{V})$ is isomorphic to $\mathrm{GL}_{n}(\mathbb{C}(X))$. Let $\boldsymbol{\Lambda}_{x}^{0}=\boldsymbol{\Lambda}_{x} \cap \mathscr{H}_{0}$ be the set of trivial bundles in the coset $\boldsymbol{\Lambda}_{x}$. The subgroup $G_{x} \subset G$ of automorphisms of $\Gamma(X, \mathscr{V})$ that leave $\Lambda_{x}^{0}$ globally invariant is called the group of monopole gauge transforms at $x$. Each element of $G_{X}$ sends a trivial sheaf $\mathscr{F}$ to a trivial sheaf $\widetilde{\mathscr{F}}_{\text {such }}$ that $\mathscr{F}_{\mid X \backslash\{x\}}=\widetilde{\mathscr{F}}_{\mid X \backslash\{x\}}$. An element of $G_{X}$ modifies at most the stalk $\mathscr{F}_{x}$.

\section{Lattices and the affine building of $\mathrm{SL}_{n}$}

2.1. Flags and filtrations. Let $V$ be a vector space over a field $\mathbb{K}$ of characteristic 0 . Given a flag $\boldsymbol{F}$ of vector spaces $0=F_{0} \subset F_{1} \subset \cdots \subset F_{s}=V$, with length $|\boldsymbol{F}|=s$ and signature $\sigma(\boldsymbol{F})=\left(n_{1}, \ldots, n_{S}\right)$ where $n_{i}=\operatorname{dim}_{\mathbb{}}\left(F_{i} / F_{i-1}\right)$, a map $u \in \operatorname{End}_{\mathbb{}}(V)$ stabilizes the flag $\boldsymbol{F}$ if $u\left(F_{i}\right) \subset F_{i}$ for all $0 \leqslant i \leqslant|\boldsymbol{F}|$. Let $\mathfrak{F l}(V)$ denote the set of flags of $V$, and $\mathfrak{F l}_{u}(V)$ the subset of flags that are stabilized by $u$. A flag $\boldsymbol{F}^{\prime}$ is transversal to $\boldsymbol{F}$ if $\left|\boldsymbol{F}^{\prime}\right|=|\boldsymbol{F}|=s$ and $F_{i}^{\prime} \oplus F_{s-i}=V$ for $1 \leqslant i \leqslant s$. In this case, the signature of $\boldsymbol{F}^{\prime}$ is equal to $\sigma^{-}=\left(n_{s}, \ldots, n_{1}\right)$. The left action of the permutation group $S_{n}$ on a sequence $a=\left(a_{1}, \ldots, a_{n}\right)$ is given by $\tau a=\left(a_{\tau(1)}, \ldots, a_{\tau(n)}\right)$ for $\tau \in S_{n}$. Let $s_{i}$ be the transposition $(i, i+1)$ exchanging $i$ and $i+1$. The set $S=\left\{s_{1}, \ldots, s_{n-1}\right\}$ makes $\left(S_{n}, S\right)$ into a Coxeter group of type $A_{n-1}$.

Let $(e)$ be a basis of $V$, and let $\sigma=\left(n_{1}, \ldots, n_{s}\right)$ be a signature. Let $\boldsymbol{F}^{\sigma}(e)$ be the flag with elements $F_{i}^{\sigma}(e)=\left\langle e_{1}, \ldots, e_{v_{i}}\right\rangle$ where $v_{i}=n_{1}+\cdots+n_{i}$. The basis $(e)$ is said to be adapted to $\boldsymbol{F}$ if any element of the flag is spanned by a subfamily of $(e)$, strictly adapted if $\boldsymbol{F}=F^{\sigma}(e)$, and transversal to $\boldsymbol{F}$ if $F_{i} \oplus\left\langle e_{\nu_{i}+1}, \ldots, e_{n}\right\rangle=V$ for all $i$. The parabolic subgroup stabilizing a flag $\boldsymbol{F}$ (for the action on a strictly adapted basis) is the subgroup $W_{\boldsymbol{F}}=\left\langle s_{i} \mid i \in I\right\rangle$ of $S_{n}$ generated by the generators corresponding to the missing dimensions $I=[n] \backslash\left\{\operatorname{dim}_{\mathbb{K}} F_{i} \mid 1 \leqslant i \leqslant n\right\}$. These properties depend in fact only on the $\mathbb{K}$-vector subspaces spanned by the vectors of (e). The opposite flag $\boldsymbol{F}^{-(e)}$ is then defined as the unique flag transversal to $\boldsymbol{F}$ for which $(e)$ is adapted. This last notion does not even depend on the order in which the vectors of $(e)$ are taken. A flag $\boldsymbol{F}^{\prime}$ is transversal to $\boldsymbol{F}$ if and only if there is a basis $(e)$ of $V$ strictly adapted to $\boldsymbol{F}$ such that $\boldsymbol{F}^{\prime}=\boldsymbol{F}^{-(e)}$. A $\mathbb{K}$-frame is an unordered set $\Phi=\left\{L_{1}, \ldots, L_{n}\right\}$ of one-dimensional $\mathbb{K}$-vector subspaces of $V$ such that $L_{1}+\cdots+L_{n}=V$. The notions defined in the previous paragraph make sense for a frame (with a fixed order on the lines for some of them). The relative position $\rho\left(\boldsymbol{F}, \boldsymbol{F}^{\prime}\right) \in S_{n}$ of two flags $\boldsymbol{F}$ and $\boldsymbol{F}^{\prime}$ is the ${ }^{2}$ permutation $\tau \in S_{n}$ such that

\footnotetext{
${ }^{2}$ Strictly speaking, $\rho$ is only unique when both flags are complete. Otherwise, we shall a bit imprecisely consider $\rho$ either as its double coset in $W_{\boldsymbol{F}^{\prime}} \rho W_{\boldsymbol{F}}$ modulo the parabolic subgroups attached to $\boldsymbol{F}$ and $\boldsymbol{F}^{\prime}$, or to the unique minimal length representative of this coset (or possibly even to any such representative).
} 
there exists a basis $(e)$ strictly adapted to $\boldsymbol{F}$ for which $(\tau e)=\left(e_{\tau(1)}, \ldots, e_{\tau(n)}\right)$ is strictly adapted to $\boldsymbol{F}^{\prime}$.

Similarly, a flag $\boldsymbol{S}$ of length $s$ in $[n]=\{1, \ldots, n\}$ is an increasing sequence $\boldsymbol{S}: S_{0}=\varnothing \subset S_{1} \subset \cdots \subset S_{S}=[n]$ of subsets of $[n]$, whose signature is the sequence $\sigma(\boldsymbol{S})=\left(n_{1}, \ldots, n_{S}\right)$ where $n_{i}=\left|S_{i}\right|-\left|S_{i-1}\right|$. For a given signature $\sigma=\left(n_{1}, \ldots, n_{s}\right)$, the standard ascending flag $\boldsymbol{S}^{\nearrow}(\sigma)$ of signature $\sigma$ is the flag composed of initial segments of $[n]$ of lengths $n_{1}+\cdots+n_{i}$ for $1 \leqslant i \leqslant s$.

Given a sequence $D=\left(d_{1}, \ldots, d_{n}\right) \in \mathbb{Z}^{n}$, let $D^{\nearrow}$ be the sequence of the elements of $D$ arranged in increasing order. Define the ascendent flag $S^{\nearrow}(D)$ of $D$ as the sequence of subsets of indices corresponding to blocks of equal elements of $D^{\nearrow}$. Let also $\left(\mathbb{Z}^{n}\right)^{\nearrow}$ denote the set of nondecreasing integer sequences. Finally, let

$$
D \cong D^{\prime} \quad \text { if } D^{\nearrow}=\left(D^{\prime}\right)^{\nearrow} .
$$

We denote with a ${ }^{\searrow}$ symbol all similarly defined descending quantities. Note that $D^{\searrow}=w_{0}\left(D^{\nearrow}\right)$ where the permutation $w_{0}=(n, n-1, \ldots, 1)$ is the largest element of $S_{n}$ in the Bruhat order.

We further define $D+\ell=\left(d_{1}+\ell, \ldots, d_{n}+\ell\right), D_{0}=D-\min D \in \mathbb{N}^{n}$ and $D^{0}=\max D-D \in \mathbb{N}^{n}$. Let $\operatorname{Tr} D=\sum_{i=1}^{n} d_{i}$ and $\Delta D=\max D-\min D$, and finally

$$
\|D\|=\sum_{j=1}^{n}\left(d_{j}-\frac{\operatorname{Tr} D}{n}\right)^{2} \quad \text { and } \quad i(D)=\sum_{j=1}^{n}\left(\max D-d_{j}\right) \in \mathbb{N} .
$$

We list some useful and obvious properties in the following lemma.

Lemma 4. For $D \in \mathbb{Z}^{n}$ and $\ell \in \mathbb{Z}$, we have

(i) $\Delta(D+\ell)=\Delta D$ and $i(D+\ell)=i(D)$

(ii) $\Delta(-D)=\Delta D$ and $i(-D)=i\left(D^{0}\right)=\sum_{j=1}^{n}\left(d_{j}-\min D\right)$,

(iii) $i(D)+i\left(D^{0}\right)=n \Delta D$.

An $\boldsymbol{F}$-admissible sequence is an integer sequence whose ascending flag is equal to the standard ascendent flag of signature $\sigma(\boldsymbol{F})=\left(n_{1}, \ldots, n_{s}\right)$; in more concrete terms, an integer sequence

$$
D=(\underbrace{d_{1}, \ldots, d_{1}}_{n_{1} \text { times }}, \underbrace{d_{2}, \ldots, d_{2}}_{n_{2} \text { times }}, \ldots, \underbrace{d_{s}, \ldots, d_{s}}_{n_{s} \text { times }}) \text { with } d_{1}<\cdots<d_{s} .
$$

Let $\mathbb{Z}^{n}(\boldsymbol{F})$ be the set of integer $\boldsymbol{F}$-admissible sequences, and let

$$
\Xi(V)=\left\{(\boldsymbol{F}, D) \mid \boldsymbol{F} \in \mathfrak{F}(V) \text { and } D \in \mathbb{Z}^{n}(\boldsymbol{F})\right\}
$$

be the set of $\boldsymbol{F}$-filtrations of $V$. 
2.2. Lattices. In the remainder of the section, we fix a point $x \in X$ and a coset $\Lambda \in H / \sim_{x}$. We drop the index $x$ for simplicity. The field $K=\widehat{\left({\left.M_{X}\right)_{x}}_{1}\right.}$ is local, and endowed with the discrete valuation $v=\operatorname{ord}_{x}$, whose valuation ring and maximal ideal we denote by $\mathbb{O}$ and $\mathfrak{m}$. Let $V$ be the $K$-vector space $\mathscr{V}_{x} \otimes_{\left(\mu_{X}\right)_{x}} K$ of dimension $n$.

Let $\mathscr{L}(u)$ denote the free 0 -module spanned by a family $(u)$ of vectors in $V$. An O-module $M \in V$ is a lattice if there exists a $K$-basis (e) of $V$ such that $M=\mathscr{L}(e)$. Let

$$
v_{\Lambda}(x)=\max \left\{k \in \mathbb{Z} \mid x \in \mathfrak{m}^{k} \Lambda\right\}
$$

be the natural valuation of $V$ induced by $\Lambda$. For any lattices $M \subset \Lambda$ in $V$, we define the interval $[M, \Lambda]$ as

$$
[M, \Lambda]=\{N \in \boldsymbol{\Lambda} \mid M \subset N \subset \Lambda\} .
$$

Let $\pi_{\Lambda}: \Lambda \rightarrow \bar{\Lambda}=\Lambda / \mathfrak{m} \Lambda \simeq \mathbb{C}^{n}$ denote the canonical surjection on the quotient module.

Elementary divisors. Let $z$ be a uniformizing parameter of $K$. For any two lattices $\Lambda$ and $M$ in $V$, there exists a unique increasing sequence of integers $d_{1} \leqslant \cdots \leqslant d_{n}$ (the elementary divisors of $M$ in $\Lambda$ ) and an 0 -basis $\left(e_{1}, \ldots, e_{n}\right)$ of $\Lambda$ such that $\left(z^{d_{1}} e_{1}, \ldots, z^{d_{n}} e_{n}\right)$ is a basis of $M$. Such a basis $(e)$ is called a Smith basis of $\Lambda$ for $M$. We will write them $d_{i}^{\Lambda}(M)$ if we want to specify the respective lattices, and we put

$$
\mathbf{E D}_{\Lambda}(M)=\left(d_{1}^{\Lambda}(M), \ldots, d_{n}^{\Lambda}(M)\right) .
$$

Note that $d_{1}^{\Lambda}(M)=v_{\Lambda}(M)$ and $\mathbf{E D}_{\Lambda}\left(z^{k} M\right)=\mathbf{E D}_{\Lambda}(M)+k$. Let also

$$
M_{\Lambda}=z^{-v_{\Lambda}(M)} M \quad \text { and } \quad \mu_{\Lambda}(M)=\operatorname{dim}_{\mathbb{C}} M_{\Lambda} / \mathfrak{m} \Lambda .
$$

If $P \in \mathrm{GL}_{n}(K)$ is a basis change from $\Lambda$ to $M$, the sequence $\mathbf{E D}_{\Lambda}(M)=$ $\left(d_{1}, \ldots, d_{n}\right)$ can be computed in the following manner. For a subset $I \subset[n]$ of cardinality $|I|$, let $S_{I}=\sum_{i \in I} s_{i}$, and for $|I|=|J|=k$, let $P_{I, J}$ denote the $(I, J)$-submatrix of $P$. The sequence $\left(d_{1}, \ldots, d_{n}\right)$ satisfies

$$
d_{k}=e_{k}-e_{k-1} \quad \text { where } e_{k}=\min _{|I|=|J|=k}\left\{v\left(\operatorname{det} P_{I, J}\right)\right\} \text { and } e_{0}=0 .
$$

It is convenient to be a bit more lax in the definition, and allow the elementary divisors to appear in another order. To avoid ambiguities, we will specify that $(e)$ is an ascending Smith basis of $\Lambda$ for $M$, if the vectors in $(e)$ are ordered according to $\mathbf{E D}_{\Lambda}(M)^{\nearrow}$.

We say that a matrix $P$ is $D$-parabolic if $P_{i j} \neq 0 \Rightarrow d_{i} \leqslant d_{j}$ for $1 \leqslant i, j \leqslant n$. For any commutative ring $R$, put $G_{D}(R)$ for the group of $D$-parabolic matrices of 
$\mathrm{GL}_{n}(R)$. The subgroup of $\mathrm{GL}_{n}(\mathrm{O})$ that acts on the set of Smith bases of $\Lambda$ for $M$ is the subgroup of $\mathscr{G}_{D}$-parabolic matrices

$$
\mathscr{G}_{D}=\left\{P \in \mathrm{GL}_{n}(\mathrm{O}) \mid v\left(P_{i j}\right) \geqslant d_{i}-d_{j}\right\} .
$$

Being $\mathscr{G}_{D}$-parabolic is stronger than $D$-parahoric, which only stabilizes the induced $D$-flag in $\Lambda / \mathfrak{m} \Lambda$, and weaker than $D$-parabolic, which stabilizes a $K$-flag of signature $\sigma(D)$. For any sequence $D=\left(d_{1}, \ldots, d_{n}\right)$, let $z^{D}$ be the diagonal matrix $\operatorname{diag}\left(z^{d_{1}}, \cdots, z^{d_{n}}\right)$. We will frequently use the following type of diagram:

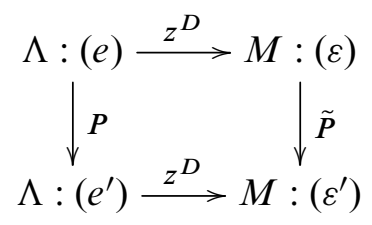

which means that $(e)$ and $\left(e^{\prime}\right)$ are two Smith bases of $\Lambda$ for $M$. Note that in this case $P \in \mathrm{GL}_{n}(\mathcal{O})$ is $\mathscr{G}_{D}$-parabolic. Since $G_{D}(\mathbb{C}) \subset \mathscr{G}_{D}$ holds, the obvious factorization $P=P_{0} P_{I}$ of a lattice gauge $P \in \mathrm{GL}_{n}(\mathcal{O})$ into a constant term $P_{0}=P(0) \in \mathrm{GL}_{n}(\mathbb{C})$ and a term $P_{I}=I+z U$ with $U \in \mathfrak{g l}_{n}(\mathcal{O})$ tangent to $I$ satisfies the property

$$
P \in \mathscr{G}_{D} \Longleftrightarrow\left(P \in G_{D}(\mathbb{C}) \text { and } P_{I} \in \mathscr{G}_{D}\right) \text {. }
$$

We can therefore usually assume that $P$ is tangent to $I$. Note that this also holds for a right factorization $P=P_{I}^{\prime} P_{0}$.

Sometimes, we will find it more convenient to consider the elementary divisors with their multiplicities. In this case, we will put $\boldsymbol{d}_{1}, \ldots, \boldsymbol{d}_{s}$ for the distinct elementary divisors of $M$ in $\Lambda$ and let $n_{i}$ be their respective multiplicities. The set $[n]=\{1, \ldots, n\}$ of indices of ordinary (simple) elementary divisors is partitioned into the subsets $I_{j}$ corresponding to a single value of the elementary divisors:

$$
I_{j}=\left\{1 \leqslant \ell \leqslant n \mid d_{\ell}=\boldsymbol{d}_{j}\right\} \text { for } 1 \leqslant j \leqslant s .
$$

2.3. Relative flag of a lattice. Any lattice $M$ induces a natural flag in $\bar{\Lambda}=\Lambda / \mathfrak{m} \Lambda$. For any $k \in \mathbb{Z}$, let

$$
M_{k}=\left(\mathfrak{m}^{-k} M \cap \Lambda\right)+\mathfrak{m} \Lambda \in[\mathfrak{m} \Lambda, \Lambda] .
$$

Lemma 5. Let $\Lambda, M$ be lattices in $V$. Let $\boldsymbol{d}_{1}, \ldots, \boldsymbol{d}_{s}$ be the distinct elementary divisors of $M$ in $\Lambda$. The flag in $\bar{\Lambda}$

$$
\boldsymbol{F}^{\Lambda}(M): 0 \subset \pi_{\Lambda}\left(M_{\boldsymbol{d}_{1}}\right) \subset \cdots \subset \pi_{\Lambda}\left(M_{\boldsymbol{d}_{s}}\right) \subset \bar{\Lambda}
$$

has signature $\sigma\left(\mathbf{E D}_{\Lambda}(M)\right)$.

Proof. Let (e) be a basis of elementary divisors of $\Lambda$ for $M$, and $I=\{1 \leqslant i \leqslant$ $\left.n \mid d_{i} \leqslant k\right\}$. Then $M_{k}$ admits $(u)$ as basis where $u_{i}=e_{i}$ if $i \in I$ and $u_{i}=z e_{i}$ if $i \notin I$. 
The spaces $M_{k}$ are thus embedded lattices, all belonging to the interval $[\mathfrak{m} \Lambda, \Lambda]$, so they take at most $n+1$ different values. Their images $\bar{M}_{k}=\pi_{\Lambda}\left(M_{k}\right)$ in the quotient space $\bar{\Lambda}$ form a flag $\boldsymbol{F}^{\Lambda}(M)$, and it is clear that $\bar{M}_{k-1} \subsetneq \bar{M}_{k}$ if and only if $k$ is an elementary divisor of $M$. If $\boldsymbol{d}_{1}, \ldots, \boldsymbol{d}_{s}$ are the distinct elementary divisors of $M$ in $\Lambda$, with multiplicities $n_{i}$, the subset of indices corresponding to $\boldsymbol{d}_{j}$ can be written as

$$
I_{j}=\left[n_{1}+\cdots+n_{j}, n_{1}+\cdots+n_{j+1}-1\right] .
$$

The lattices $M_{k}, M_{\ell}$ coincide if and only if there exists $i$ such that $\boldsymbol{d}_{i} \leqslant k, \ell<\boldsymbol{d}_{i+1}$ (with the conventions $\boldsymbol{d}_{0}=-\infty$ and $\boldsymbol{d}_{s+1}=+\infty$ ). Therefore the flag $\boldsymbol{F}^{\Lambda}(M)$ has exactly length $s$, and its signature is equal to the sequence $\left(n_{1}, \ldots, n_{s}\right)$.

The components of $\boldsymbol{F}^{\Lambda}(M)$ can be indexed either as $\bar{M}_{\boldsymbol{d}_{i}}$, by the value of the elementary divisor $\boldsymbol{d}_{i}$ to which it is attached (if known), or as $\bar{M}_{i}$, by its index in the flag (here $i$ ). In this latter case, we will also use the notation $F_{i}^{\Lambda}(M)$. It will hopefully be always clear which convention we are using.

Lemma 6. Let $\Lambda, M, N$ be lattices in $V$. Let $D=\mathbf{E D}_{\Lambda}(M)^{\nearrow}$ and $D^{\prime}=$ $\mathbf{E D}_{\Lambda}(N)^{\nearrow}$. If either

(i) there exists a common Smith basis for $\Lambda, M$ and $N$, or

(ii) the flags $\boldsymbol{F}^{\Lambda}(N)$ and $\boldsymbol{F}^{\Lambda}(M)$ are transverse,

then we have

$$
\mathbf{E D}_{M}(N) \cong D^{\prime}-\sigma D,
$$

where $\sigma=\rho\left(\boldsymbol{F}^{\Lambda}(N), \boldsymbol{F}^{\Lambda}(M)\right)$ is the relative position of the induced flags in $\Lambda / \mathfrak{m} \Lambda$.

A similar formula holds for the descending sequences $D \searrow$, but with $w_{0} \sigma w_{0}$ instead of $\sigma$.

Proof. We summarize the setting by means of the following scheme:

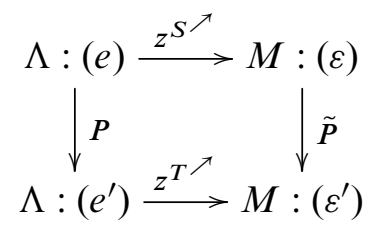

If there is an apartment containing $\Lambda, M, N$, then one can assume that $P$ is a permutation matrix, namely $\Pi_{\sigma^{-1}}$. In the second case, it is possible to choose $P=$ $\Pi_{w_{0}}(I+z U)$ with $U \in \mathfrak{g l}(\mathcal{O})$. Let $Q=I+z U$. Then we have $\tilde{P}_{i j}=Q_{i j} z^{t_{j}-s_{n+1-i}}$. According to formula (2), we have $d_{1}=\min _{1 \leqslant i, j \leqslant n}\left(v\left(P_{i j}\right)+t_{j}-s_{n+1-i}\right)$. The minimum of $t_{j}-s_{n+1-i}$ is attained for $(i, j)=(1,1)$, and by assumption we have $v\left(P_{11}\right)=0$. Therefore, we get $d_{1}=t_{1}-s_{n}$. Let us prove by induction that $d_{i}=t_{i}-s_{n+1-i}$ for all $i$. Assume that $e_{i}=T_{\{1, \ldots, i\}}-S_{\{n+1-i, \ldots, n\}}$ for $i \leqslant k-1$. 
Formula (2) yields $e_{k}=\min _{|I|=|J|=k}\left\{v\left(\operatorname{det} P_{I, J}\right)+T_{J}-\left(w_{0} S\right)_{I}\right\}$. The minimum for $T_{J}-\left(w_{0} S\right)_{I}$ is attained for $I=J=\{1, \ldots, k\}$, while by assumption the principal minor $P_{I, I}$ has valuation 0 . Hence $e_{k}=T_{[k]}-\left(w_{0} S\right)_{[k]}$ and thus $d_{k}=$ $e_{k}-e_{k-1}=t_{k}-s_{n+1-k}$ for all $k$.

For a flag $\boldsymbol{F}=\left(\boldsymbol{F}_{i}\right)_{1 \leqslant i \leqslant s}$ of $K$-vector spaces, and a lattice $\Lambda$, let $\boldsymbol{F}_{\Lambda}=$ $(\boldsymbol{F} \cap \Lambda) / \mathfrak{m} \Lambda$ be the flag $\left(\left(\boldsymbol{F}_{i} \cap \Lambda\right) / \mathfrak{m} \Lambda\right)_{1 \leqslant i \leqslant s}$ induced in $\Lambda / \mathfrak{m} \Lambda$. The following result is easily established.

Lemma 7. Let $\boldsymbol{F}$ and $\boldsymbol{F}^{\prime}$ be two $K$-flags of $V$. For any basis (e), adapted for $\boldsymbol{F}$ and $\boldsymbol{F}^{\prime}$, one has

$$
\rho\left(\boldsymbol{F}, \boldsymbol{F}^{\prime}\right)=\rho\left(\boldsymbol{F}_{\Lambda}, \boldsymbol{F}_{\Lambda}^{\prime}\right), \quad \text { where } \Lambda=\mathscr{L}(e) .
$$

2.4. The affine building of $\mathrm{SL}(\boldsymbol{V})$. For this section, good references are [Garrett 1997; Ronan 1989], and especially [Abramenko and Brown 2008]. The affine building $B_{n}$ naturally attached to $\operatorname{SL}(V)$ is the following $(n-1)$-dimensional simplicial complex. Two lattices $\Lambda$ and $M$ are homothetic if there exists $\alpha \in K^{*}$ such that $M=\alpha \Lambda$. Let $[\Lambda]$ be the homothety class of the lattice $\Lambda$ in $V$. Two classes $L$ and $L^{\prime}$ are adjacent if and only if there exist representatives $\Lambda$ of $L$ and $M$ of $L^{\prime}$ such that $\mathfrak{m} \Lambda \subset M \subset \Lambda$. Consider a graph whose vertices are the homothety classes of lattices in $V$, and edges connect all adjacent vertices. The affine building $B_{n}$ is the flag simplicial complex associated with this graph, or in other terms, its clique complex. A simplex $A$ is a set $\left\{L_{1}, \ldots, L_{k}\right\}$ of mutually adjacent vertices, and the face relation $B \leqslant A$ is defined by the inclusion $B \subset A$ of these sets.

Lemma 8. Let $L, L^{\prime}, L^{\prime \prime}$ be vertices in $B_{n}$. If $L^{\prime}$ and $L^{\prime \prime}$ are adjacent, then for any representatives $\Lambda \in L, M \in L^{\prime}$ and $N \in L^{\prime \prime}$, the flags $\boldsymbol{F}^{\Lambda}(M)$ and $\boldsymbol{F}^{\Lambda}(N)$ are compatible (in the sense that their components are all pairwise comparable). In particular, a maximal simplex $C$ induces a complete flag in $\Lambda / \mathfrak{m} \Lambda$.

Proof. One can find representatives $\Lambda \in L, M \in L^{\prime}$ and $N \in L^{\prime \prime}$, and a basis (e) of $\Lambda$ such that $M=\mathscr{L}\left(z^{D} e\right)$ and $N=\mathscr{L}\left(z^{D^{\prime}} e\right)$, and that $D^{\prime}-D \in\{0,1\}^{n}$. Indeed, let $(u)$ be a basis of $M / \mathfrak{m} M$ which is adapted to both flags $\boldsymbol{F}^{M}(\Lambda)$ and $\boldsymbol{F}^{M}(N)$. There exists a lifting $(\varepsilon)$ of $(u)$ in $M$ which is a Smith basis of $M$ for $\Lambda$. Since $M$ and $N$ are adjacent, any lifting of $(u)$ is a Smith basis for $N$. Then $(e)=\left(z^{\mathbf{E D}_{M}(\Lambda)} \varepsilon\right)$ is a common Smith basis of $\Lambda$ for $M$ and $N$. By definition of adjacency, one can ensure that the representatives satisfy $\mathfrak{m} M \subset N \subset M$; that is, $D^{\prime}-D \in\{0,1\}^{n}$. Let now $I_{k}=\left\{1 \leqslant i \leqslant n \mid d_{i} \leqslant k\right\}$ and $I_{k}^{\prime}=\left\{1 \leqslant i \leqslant n \mid d_{i}^{\prime} \leqslant k\right\}$. Then we have $I_{k-1} \subset I_{k}^{\prime} \subset I_{k}$ for all $i$. Since $\boldsymbol{F}^{\Lambda}(M)$ is spanned by the subfamilies $\left(\overline{e_{i}}\right)_{i \in I_{k}}$ for $k \in \mathbb{Z}$, the claim follows. 
Simplices and chambers. A maximal simplex, or chamber in $B_{n}$, is an $n$-chain $C$ of vertices $L_{0}, \ldots, L_{n-1}$ with representatives $\Lambda_{i}$ for $0 \leqslant i \leqslant n-1$ satisfying

$$
\mathfrak{m} \Lambda_{0} \subset \Lambda_{1} \subset \cdots \subset \Lambda_{n-1} \subset \Lambda_{0} .
$$

A basis (e) is a fundamental basis for $C$ at $L_{i}$ if

$$
L_{(i+j) \bmod n}=\left[\mathscr{L}\left(e_{1}, \ldots, e_{j}, z e_{j+1}, \ldots, z e_{n}\right)\right] \text { for } 0 \leqslant j \leqslant n-1 .
$$

Lemma 9. The set of chambers which contain a given vertex $L$ is in bijection with the set of complete flags in $\Lambda / \mathfrak{m} \Lambda$ with $\Lambda \in L$. A basis (e) is fundamental for $C$ at $L$ if and only if its image in $\Lambda / \mathfrak{m} \Lambda$ is strictly adapted to the flag $\boldsymbol{F}^{\Lambda}(C)$.

A (partial) flag $\boldsymbol{F}$ in $\Lambda / \mathfrak{m} \Lambda$ can be lifted (by $\pi_{[\Lambda]}^{-1}$ ) to a uniquely defined simplex in $B_{n}$ containing the vertex $L=[\Lambda]$.

Definition 10. The graph-theoretic distance, canonical metric and index on $B_{n}$ are defined, respectively, by

$d\left(L, L^{\prime}\right)=\Delta\left(\mathbf{E D}_{\Lambda}\left(\Lambda^{\prime}\right)\right), \quad \boldsymbol{d}\left(L, L^{\prime}\right)=\left\|\mathbf{E D}_{\Lambda}\left(\Lambda^{\prime}\right)\right\|, \quad\left[L: L^{\prime}\right]=i\left(\mathbf{E D}_{\Lambda}\left(\Lambda^{\prime}\right)^{0}\right)$ for any representatives $\Lambda, \Lambda^{\prime}$ of $L, L^{\prime}$.

Note that $d\left(L, L^{\prime}\right)=-v_{\Lambda}\left(\Lambda^{\prime}\right)-v_{\Lambda^{\prime}}(\Lambda)$ also holds, and that these three maps are indeed symmetric. The $\boldsymbol{d}$ metric makes the geometric realization of $B_{n}$ into a CAT(0) space [Abramenko and Brown 2008, Theorem 11.16, p. 555].

Apartments. Let $\Phi=\left\{d_{1}, \ldots, d_{n}\right\}$ be a $K$-frame of $V$. The set

$$
\mathscr{A}_{\Phi}=\left\{\Lambda=\ell_{1}+\cdots+\ell_{n} \mid \ell_{i} \text { is a lattice in } d_{i}\right\}
$$

of lattices spanned over multiples of the vectors in $\Phi$ induces a simplicial subcomplex in the affine building $B_{n}$ called the apartment spanned by $\Phi$. For any lattice $\Lambda \in \boldsymbol{\Lambda}$, a $\Lambda$-basis of the apartment $\mathscr{A}_{\Phi}$ is a collection $(e)=\left(e_{1}, \ldots, e_{n}\right)$ of vectors such that $e_{i}$ spans $d_{i}$ and $v_{\Lambda}\left(e_{i}\right)=0$. Such a family is unique up to permutation and to multiplication of each $e_{i}$ by a scalar $\lambda_{i} \in 0^{*}$. The lattice is an element of the apartment $\mathscr{A}_{\Phi}$ if and only if the family $(e)=\left(e_{1}, \ldots, e_{n}\right)$ is actually a basis of the lattice $\Lambda$. Equivalently, and without reference to a basis, this means that

$$
\Lambda=\bigoplus_{i=1}^{n} \Lambda \cap d_{i} .
$$

In the general case, the lattice $\Lambda_{\Phi}=\bigoplus_{i=1}^{n} \Lambda \cap d_{i}$ is the largest sublattice of $\Lambda$ in the apartment $\mathscr{A}_{\Phi}$. The homothety class $L_{\Phi}=\left[\Lambda_{\Phi}\right]$ is therefore the closest point projection of $L=[\Lambda]$ on $\mathscr{A}_{\Phi}$, and the map

$$
\begin{aligned}
\rho_{\Phi}: B_{n} & \rightarrow \mathscr{A}_{\Phi} \\
\Lambda & \mapsto \Lambda_{\Phi}
\end{aligned}
$$


is the retract onto $\mathscr{A}_{\Phi}$.

Galleries and type. Two chambers $C$ and $C^{\prime}$ are adjacent if they share $n-1$ vertices in common (a so-called panel). A gallery $\Gamma$ from $C$ to $C^{\prime}$ of length $\ell(\Gamma)=\ell$ is a sequence of chambers $\Gamma=\left(C_{0}=C, C_{1}, \ldots, C_{\ell}=C^{\prime}\right)$ such that $C_{i}$ is adjacent to $C_{i+1}$. The gallery distance is defined as

$$
\ell(C, D)=\min \{\ell(\Gamma) \mid \Gamma \text { is a gallery from } C \text { to } D\} .
$$

The vertices in the building $B_{n}$ can be labelled by $\mathbb{Z} / n \mathbb{Z}$, which we assume given from now on. Let $S=\left\{s_{0}, \ldots, s_{n-1}\right\}$. The affine Weyl group $W$ of the building then has a presentation

$$
\left.W=\langle S| s_{i}^{2}=1 \text { and }\left(s_{i} s_{i+1}\right)^{3}=1\right\rangle,
$$

where the indices are understood modulo $n$. Every chamber has exactly one vertex labelled $i$, that we denote $M_{i}(C)$, for every $i \in \mathbb{Z} / n \mathbb{Z}$. Two chambers are called $i$-adjacent when their common panel precisely does not contain those vertices labelled $i$. Two $i$-adjacent chambers $C$ and $C^{\prime}$ are then said to have $W$-distance $\delta\left(C, C^{\prime}\right)=s_{i}$. If $\Gamma=\left(C, C_{1}, \ldots, C_{t-1}, C^{\prime}\right)$ is any gallery from $C$ to $C^{\prime}$ lying inside an apartment, the $W$-distance $\delta\left(C, C^{\prime}\right)$ is defined as the product

$$
\delta\left(C, C^{\prime}\right)=\delta\left(C, C_{1}\right) \cdots \delta\left(C_{t-1}, C^{\prime}\right) .
$$

There is a bijection between minimal galleries between $C$ and $C^{\prime}$ and minimal length decompositions of the $W$-distance $\delta\left(C, C^{\prime}\right) \in W$ into products of generators in $S$. Let $\mathbb{Z}_{0}^{n}$ be the set of $n$-tuples of integers summing to 0 . A labelling of the vertices by $\mathbb{Z} / n \mathbb{Z}$ induces an isomorphism

$$
W \stackrel{\phi}{\simeq} S_{n} \rtimes \mathbb{Z}_{0}^{n} \quad \text { given by }\left\{\begin{array}{l}
\phi\left(s_{i}\right)=((i, i+1), 0) \quad \text { for } 1 \leqslant i \leqslant n, \\
\phi\left(s_{0}\right)=((1, n),(1,0, \ldots, 0,-1)) .
\end{array}\right.
$$

Let $\delta=\delta\left(C, C^{\prime}\right)$ be the $W$-distance between two chambers $C$ and $C^{\prime}$. Let $L=M_{0}(C)$ and $L^{\prime}=M_{0}\left(C^{\prime}\right)$ be the respective unique vertices of type 0 of $C$ and $C^{\prime}$. Then $\phi(\delta)=(\sigma, K)$ is the unique couple such that there exists a fundamental basis $(e)$ of $C$ at $L$ for which $\left(z^{K} e_{\sigma}\right)=\left(z^{k_{1}} e_{\sigma(1)}, \ldots, z^{k_{n}} e_{\sigma(n)}\right)$ is a fundamental basis of $C^{\prime}$ at $L^{\prime}$.

Walls. Let (e) be a basis of $V$, and let $\mathscr{A}$ be the apartment spanned by $(e)$. In the basis (e), any lattice $L$ in $\mathscr{A}$ can be represented by a unique (up to an integer multiple of $(1, \ldots, 1)) n$-tuple $\left(x_{1}(L), \ldots, x_{n}(L)\right) \in \mathbb{Z}^{n}$. The set $\mathscr{H}$ of walls of $V$ for $W$ is the set of hyperplanes

$$
H_{i, j}^{(k)}=\left\{x \in \mathbb{R}^{n} \mid x_{i}-x_{j}=k\right\} \quad \text { for } 1 \leqslant i<j \leqslant n \text { and } k \in \mathbb{Z} .
$$

Define the corresponding half-spaces 


$$
\begin{aligned}
H_{i, j}^{(k)+} & =\left\{x \in \mathbb{R}^{n} \mid x_{i}-x_{j} \geqslant k\right\}, \\
H_{i, j}^{(k)-} & =\left\{x \in \mathbb{R}^{n} \mid x_{i}-x_{j} \leqslant k\right\} .
\end{aligned}
$$

For a hyperplane $H \in \mathscr{H}$ and a simplex $A$, let

$$
\sigma_{H}(A)= \begin{cases}+ & \text { if } A \subset H^{+} \backslash H^{-}, \\ - & \text {if } A \subset H^{-} \backslash H^{+}, \\ 0 & \text { if } A \subset H^{+} \cap H^{-} .\end{cases}
$$

A wall $H$ separates the simplices $A$ and $B$ if $\sigma_{H}(A) \sigma_{H}(B)=-$ for the usual sign product rule. Any simplex $A$ is completely defined by its sign sequence $\left(\sigma_{H}(A)\right)_{H \in \mathscr{H}}$. However, there is only a finite number of them which are relevant (i.e., whose defining equations or inequalities are not redundant). If we define the fundamental chamber as $C_{0}=\left(L_{0}, \ldots, L_{n-1}\right)$, where $L_{i}=\left[\mathscr{L}\left(e_{1}, \ldots, e_{i}\right.\right.$, $\left.\left.z e_{i+1}, \ldots, z e_{n}\right)\right]$, the (essential) walls of $C_{0}$ are the hyperplanes $H_{i,(i \bmod n)+1}^{(0)}$ for $1 \leqslant i \leqslant n$, and its sign sequence is

$$
\sigma_{H_{i,(i \bmod n)+1}^{(k)}}\left(C_{0}\right)= \begin{cases}+ & \text { for } 1 \leqslant i \leqslant n-1 \text { and } k \leqslant 0 \text { or } i=n \text { and } k \geqslant 0, \\ - & \text { for } 1 \leqslant i \leqslant n-1 \text { and } k \geqslant 0 \text { or } i=n \text { and } k \leqslant 0 .\end{cases}
$$

Definition 11. Let $A, B$ be simplices in $B_{n}$. The gallery distance $\ell(A, B)$ is defined as $\min _{A \leqslant C, B \leqslant D} \ell(C, D)$.

Lemma 12. For two simplices $A, B$ we have

$$
\ell(A, B)=\left|\left\{H \in \mathscr{H} \mid \sigma_{H}(A) \sigma_{H}(B)=-\right\}\right| .
$$

If $A=[\Lambda]$ and $B=[M]$ are vertices, we have

$$
\ell(A, B)=\sum_{1 \leqslant i<j \leqslant n} \max \left(0, d_{j}-d_{i}-1\right), \quad \text { where }\left(d_{1}, \ldots, d_{n}\right)=\mathbf{E D}_{\Lambda}(M)^{\nearrow}
$$

Proof. The first assertion is known (see [Abramenko and Brown 2008, p. 32]). Take a basis ( $e$ ) of $V$ where $A$ has coordinates $a$ and $B$ coordinates $b$. The description (3) of walls shows that $A$ and $B$ are separated by $H_{i, j}^{(k)}$ if and only if $a_{i}-a_{j} \leqslant k-1$ and $b_{i}-b_{j} \geqslant k+1$. Taking for (e) a Smith basis of $\Lambda$ for $M$ gives $a=0$ and $b=\left(d_{1}, \ldots, d_{n}\right)$. The result is then a straightforward count.

2.5. Forms. For $L \in B_{n}$, let $\bar{L}=\Lambda / \mathfrak{m} \Lambda \simeq \mathbb{C}^{n}$ for $\Lambda \in L$. This definition is independent of the choice of $\Lambda$ as there is a canonical isomorphism between $\Lambda / \mathfrak{m} \Lambda$ and $\mathfrak{m}^{k} \Lambda / \mathfrak{m}^{k+1} \Lambda$ for any $k$. For $L^{\prime} \in B_{n}$, let $N$ be the unique representative of $L^{\prime}$ such that $v_{\Lambda}(N)=0$, and define

$$
\pi_{L}\left(L^{\prime}\right)=(N+\mathfrak{m} \Lambda) / \mathfrak{m} \Lambda \in \bar{L} .
$$

The map $\pi_{L}$ induces an isomorphism of simplicial complexes between the link $\operatorname{lk}(L)$ of $L=[\Lambda]$ and the set $E$ of chains of linear subspaces of $\bar{L}$. A form in a lattice $\Lambda$ is 
a $\mathbb{C}$-vector subspace $Y$ of $\Lambda$ spanned by an 0 -basis (e) of $\Lambda$. For any $x \in \Lambda$, there is a unique representative $x_{Y}$ of the $\operatorname{coset} x+\mathfrak{m} \Lambda$ in $Y$. This induces a well-defined isomorphism

$$
\phi_{Y}: Y \stackrel{\simeq}{\longrightarrow} \Lambda / \mathfrak{m} \Lambda .
$$

We will say that $Y \subset \Lambda$ is a Smith form for $M$ if there is a basis of $Y$ which is a Smith basis of $\Lambda$ for $M$.

Lemma 13. Let $\Lambda$ be a lattice in $V$ and $Y$ be a form in $\Lambda$.

(i) For any basis (e) of the lattice $\Lambda$, there exists a unique $\mathbb{C}$-basis $\left(e_{Y}\right)$ of the form $Y$ whose image in $\Lambda / \mathfrak{m} \Lambda$ coincides with the image of $(e)$. We call $\left(e_{Y}\right)$ the $Y$-basis of $(e)$.

(ii) Given a filtration $(\boldsymbol{F}, D)$ in $\Xi(\Lambda / \mathfrak{m} \Lambda)$, there exists a unique lattice $M=$ $\mathscr{L}_{Y}(\boldsymbol{F}, D)$ such that $\boldsymbol{F}^{\Lambda}(M)=\boldsymbol{F}$ and $\mathbf{E D}_{\Lambda}(M)=D$ that admits a Smith basis in $Y$.

(iii) For any lattice $M$ such that $d(\Lambda, M)=1$, let $(\bar{e})$ a basis of $\Lambda / \mathfrak{m} \Lambda$ respecting $\pi_{\Lambda}(M)=M / \mathfrak{m} \Lambda$. Then the $Y$-basis $\left(e_{Y}\right)$ is a Smith basis for $M$.

Proof. The basis $(\varepsilon)$ obtained by putting $\varepsilon_{i}=\left(e_{i}\right)_{Y}=\phi_{Y}^{-1}\left(\pi_{\Lambda}\left(e_{i}\right)\right)$ obviously satisfies the conditions of (i). For any $\mathbb{C}$-basis (e) of $Y$ which respects the flag $\boldsymbol{F}$, put $M=\bigoplus_{i=1}^{n} z^{d_{i}} e_{i}$. Let ( $\left.\tilde{e}\right)$ be another basis of $Y$ and $\tilde{M}=\bigoplus_{i=1}^{n} z^{d_{i}} \tilde{e}_{i}$. The matrix of the change of basis from $\left(z^{D} e\right)$ to $\left(z^{D} \tilde{e}\right)$ is equal to $P=z^{D} C z^{-D}$, where $C \in \mathrm{GL}_{n}(\mathbb{C})$ is the matrix of the change of basis from $(e)$ to $(\tilde{e})$. By definition of the parabolic subgroup $P_{\boldsymbol{F}}$, one has $z^{D} C z^{-D} \in \mathrm{GL}_{n}(\mathcal{O}) \Longleftrightarrow C \in P_{\boldsymbol{F}}$; hence $M=\tilde{M}$ if and only if (e) and ( $\tilde{e})$ both respect the flag $\boldsymbol{F}$. Note that the gauge from the basis (e) to its $Y$-basis is always of the form $P=I+z U \in \mathrm{GL}_{n}(\mathrm{O})$. Let $W=\pi_{\Lambda}(M)$ and let

$$
T=\left(\begin{array}{cc}
0 r & 0 \\
0 & I_{n-r}
\end{array}\right)
$$

be the elementary divisors of $M_{\Lambda}=z^{-v_{\Lambda}(M)} M$ with respect to $\Lambda$. Assume that (e) satisfies the assumptions of (iii). Then the $Y$-basis $\left(e_{Y}\right)$ is obtained by a gauge $P=I+z U \in \mathrm{GL}_{n}(\mathrm{O})$. Putting

$$
U=\left(\begin{array}{ll}
U_{11} & U_{12} \\
U_{21} & U_{22}
\end{array}\right)
$$

we have

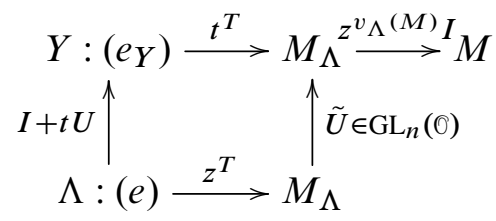


since

$$
\tilde{U}=z^{-T}(I+z U) z^{T}=\left(\begin{array}{cc}
I_{r}+z U_{11} & z^{2} U_{12} \\
U_{21} & I_{n-r}+z U_{22}
\end{array}\right) .
$$

The basis $\left(e_{Y}\right)$ is therefore indeed a Smith basis of $\Lambda$ for $M$.

For any two forms $Y$ and $\tilde{Y}$ in $\Lambda$, the set of gauges between bases of $Y$ and $\tilde{Y}$ is an element of the double coset $\mathrm{GL}_{n}(\mathbb{C}) \backslash \mathrm{GL}_{n}(\mathbb{O}) / \mathrm{GL}_{n}(\mathbb{C})$. Let $z$ be a uniformizing parameter. With the convention that $\operatorname{deg}_{z} P=\infty$ if $P \in \mathrm{GL}_{n}(\mathbb{O}) \backslash \mathfrak{g l}(\mathbb{C}[z])$, the following definition makes sense.

Definition 14. Let $Y, \tilde{Y}$ be two forms in $\Lambda$. Define the $z$-distance as

$$
\delta_{z}(Y, \tilde{Y})=\min \left(\operatorname{deg}_{z} P, \operatorname{deg}_{z} P^{-1}\right) \in \mathbb{N} \cup\{\infty\}
$$

for any gauge $P$ from a basis of $Y$ to a basis of $\tilde{Y}$.

Lemma 15. If $t=d(\Lambda, M)$, then for any form $Y$ of $\Lambda$, and any uniformizing parameter $z$, there exists a Smith form $\tilde{Y}$ for $M$ at a $z$-distance $\delta_{z}(Y, \tilde{Y}) \leqslant t-1$.

Proof. There exists a Smith form $Y^{\prime}$ of $\Lambda$ for $M$. Let $P=P_{0}+P_{1} z+\cdots \in \mathrm{GL}_{n}(0)$ be a gauge corresponding to a basis change from $Y$ to $Y^{\prime}$. Let $\bar{P}=P_{0}+\cdots+P_{k} z^{k}$ for some $k \geqslant 0$, and let $\tilde{Y}$ be the form obtained by this gauge transformation, as explained in the following scheme:

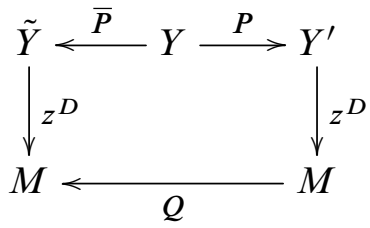

We have $Q=z^{-D} P^{-1} \bar{P} z^{D}=\left(\widetilde{P}_{i j} z^{d_{j}-d_{i}}\right)$, where $\widetilde{P}=P^{-1} \bar{P}$. By construction, we have $\widetilde{P}=P^{-1}(P-(P-\bar{P}))=I+z^{k+1} U$ with $U \in \mathfrak{g l}(\mathcal{O})$. As soon as $k \geqslant t-1$, we have $Q \in \mathrm{GL}_{n}(\mathcal{O})$; hence the form $\tilde{Y}$ is a Smith form for $M$.

2.6. Shortest paths and elementary splittings. A shortest path is a path $\Gamma$ in $B_{n}$ such that for any vertices $L, L^{\prime} \in \Gamma$, the length of the path between $L$ and $L^{\prime}$ induced by $\Gamma$ is equal to $d\left(L, L^{\prime}\right)$. For a path $\Gamma=\left(L_{0}, \ldots, L_{t}\right)$ in $B_{n}$, and a representative $\Lambda_{0} \in L_{0}$, let the $\Lambda_{0}$-normalized sequence of $\Gamma$ be the unique sequence of lattices $\Lambda_{i} \in L_{i}$ such that $v_{\Lambda_{0}}\left(\Lambda_{i}\right)=0$.

Lemma 16. Let $\Lambda, M \in \Lambda$. The $\Lambda$-normalized sequence $\left(\Lambda, \Lambda_{1}, \ldots, \Lambda_{t}=M_{\Lambda}\right)$ of a shortest path from $[\Lambda]$ to $[M]$ satisfies $\Lambda \supset \Lambda_{1} \supset \cdots \supset \Lambda_{t} \supset z^{t} \Lambda$.

Proof. Let $\Gamma=\left(\Lambda=\Lambda_{0}, \Lambda_{1}, \ldots, \Lambda_{t-1}, M=\Lambda_{t}\right)$ be a path of minimal length $t$, and let us prove that $\Lambda \supset \Lambda_{1} \supset \cdots \supset \Lambda_{t}$ by induction on $t$. For $t=1$, this is the very definition of adjacency in $B_{n}$. Assuming that the claim is established for any pair of 
lattices at distance $\leqslant t-1$, we have $M \subset \Lambda \supset \Lambda_{1} \supset \cdots \supset \Lambda_{t-1}$ for the normalized sequence of $\Gamma$. Since $\Lambda_{t-1}$ and $M$ are adjacent, there exists a unique $k \in \mathbb{Z}$ such that $z^{k} \Lambda_{t-1} \supset M \supset z^{k+1} \Lambda_{t-1}$. We know that $d\left(\Lambda, \Lambda_{t-1}\right)=t-1$, and hence we have $\Lambda \supset \Lambda_{t-1} \supset z^{t-1} \Lambda$; therefore we get

$$
z^{k} \Lambda \supset z^{k} \Lambda_{t-1} \supset M \supset z^{k+1} \Lambda_{t-1} \supset z^{t+k} \Lambda .
$$

If $k>0$, then $v_{\Lambda}(M) \geqslant k>0$, which was excluded by assumption. But if $k<0$, then $d(\Lambda, M)<t$, which is also excluded. Thus we have $k=0$, and the claim is proved.

The following result explains how to construct some shortest paths algebraically.

Proposition 17. Let $L, L^{\prime} \in B_{n}$, and let $t=d\left(L, L^{\prime}\right)$. For $k \in \mathbb{N}$, let $L_{k}=$ $\left[\Lambda^{\prime}+\mathfrak{m}^{k} \Lambda\right]$, and $M_{k}=\left[\Lambda \cap \mathfrak{m}^{k-t} \Lambda^{\prime}\right]$, where $\Lambda \in L$ and $\Lambda^{\prime} \in L^{\prime}$ are such that $v_{\Lambda}\left(\Lambda^{\prime}\right)=0$. Then $d\left(L_{k}, L_{k+1}\right)=1$ for $0 \leqslant k \leqslant t-1$ and $L_{t}=L^{\prime}$. The paths

$$
\Gamma_{\min }\left(L, L^{\prime}\right)=\left(L_{0}, L_{1}, \ldots, L_{t}\right) \quad \text { and } \quad \Gamma_{\max }\left(L, L^{\prime}\right)=\left(M_{0}, M_{1}, \ldots, M_{t}\right)
$$

are shortest paths from $L$ to $L^{\prime}$, respectively called the min- and the max-shortest path from $L$ to $L^{\prime}$. For any $\Lambda$-normalized sequence $\left(N_{0}=\Lambda, N_{1}, \ldots, N_{t}=M\right)$ of a shortest path $\Gamma^{\prime}$ from $L$ to $L^{\prime}$, one has

$$
L_{i} \subset N_{i} \subset M_{i} \quad \text { for } 0 \leqslant i \leqslant t .
$$

Proof. The existential part of the lemma is easy to verify by using Smith bases of the representatives $\Lambda \in L$ and $M \in L^{\prime}$, and is left to the reader. First note that the minand max-shortest paths are in correspondence under the duality map $\Lambda \mapsto \Lambda^{*}=$ $\operatorname{hom}_{K}(\Lambda, \mathcal{O})$, so we only need to prove claims on one type of path. Note that the shortest path interval is symmetric. Indeed, letting $\Gamma\left(L^{\prime}, L\right)=\left(L_{0}^{\prime}, L_{1}^{\prime}, \ldots, L_{t}^{\prime}\right)$, we have $L_{k}=\left[M_{\Lambda}+z^{k} \Lambda\right]$ and $L_{t-k}^{\prime}=\left[\Lambda_{M}+z^{t-k} M\right]$. By definition we have $\Lambda_{M}+z^{t-k} M=z^{-v_{M}(\Lambda)} \Lambda+z^{-v_{M}(\Lambda)-v_{\Lambda}(M)-k} M=z^{-v_{M}(\Lambda)-k}\left(z^{k} \Lambda+z^{-v_{\Lambda}(M)} M\right)$.

Therefore $L_{t-k}^{\prime}=L_{k}$.

Now we turn to the proof of $\Lambda_{i}=M+\mathfrak{m}^{i} \Lambda \subset N_{i} \subset \Lambda \cap \mathfrak{m}^{i-t} M=M_{i}$ for the shortest path $\Gamma^{\prime}=\left(N_{0}, \ldots, N_{t}\right)$, which we will prove by induction on the distance $t=d\left(L, L^{\prime}\right)$. For convenience, let any path $\left([\Lambda]=L_{0}, L_{1}, \ldots, L_{t-1},[M]=L_{t}\right)$ be represented by its $\Lambda$-normalized sequence $\left(\Lambda, \Lambda_{1}, \ldots, \Lambda_{t-1}, \Lambda_{t}=M\right)$ of lattices $\Lambda_{i} \in L_{i}$ such that $v_{\Lambda}\left(\Lambda_{i}\right)=0$. The claim is obvious for $t=1$. Let us assume it holds for any shortest path between pairs of vertices in $B_{n}$ whose distance is at most $t-1$. Suppose then that $d(\Lambda, M)=t$. Let $\Lambda=\Lambda_{0} \supset \Lambda_{1} \supset \cdots \supset \Lambda_{t-1} \supset M$ be the min-shortest path from $\Lambda$ to $M$ and let $\Lambda \supset \Lambda_{1}^{\prime} \supset \cdots \supset \Lambda_{t-1}^{\prime} \supset M$ represent the path $\Gamma$ of minimal length. By assumption, $d\left(\Lambda, \Lambda_{t-1}^{\prime}\right)=t-1$, therefore 
we have $\Lambda \supset \Lambda_{t-1}^{\prime} \supset z^{t-1} \Lambda$, and by definition, we have $\Lambda_{t-1}=M+z^{t-1} \Lambda$. Therefore, we get

$$
\begin{aligned}
\Lambda_{t-1} \cap \Lambda_{t-1}^{\prime} & =\left(M+z^{t-1} \Lambda\right) \cap \Lambda_{t-1}^{\prime} & & \\
& =M+\left(z^{t-1} \Lambda \cap \Lambda_{t-1}^{\prime}\right) & & \text { (because } \left.M \subset \Lambda_{t-1}^{\prime}\right) \\
& =M+z^{t-1} \Lambda & & \text { (since } \left.z^{t-1} \Lambda \subset \Lambda_{t-1}^{\prime}\right) \\
& =\Lambda_{t-1} . & &
\end{aligned}
$$

Thus $\Lambda_{t-1} \subset \Lambda_{t-1}^{\prime}$ holds. On the other hand, $M_{t-1}=\Lambda \cap \mathfrak{m}^{-1} M$ is by construction the largest lattice containing $M$, contained in $\Lambda$ and adjacent to $M$. Therefore $\Lambda_{t-1} \subset \Lambda_{t-1}^{\prime} \subset M_{t-1}$. By the induction assumption, the claim is established.

If $D=\left(d_{1}, \ldots, d_{n}\right)$ are the elementary divisors of $M_{\Lambda}$ in $\Lambda$, then the lattices $\Lambda_{k}$ on the (normalized) min-shortest path from $\Lambda$ to $M$ have elementary divisors $D_{k}=\left(\min \left(d_{1}, k\right), \ldots, \min \left(d_{n}, k\right)\right)$ in $\Lambda$. The differences $\Delta_{k}=D_{k}-D_{k-1}$ are the intermediate elementary divisors as explained in the following:

$$
\Lambda \stackrel{z^{\Delta_{1}}}{\longrightarrow} \Lambda_{1} \stackrel{z^{\Delta_{2}}}{\longrightarrow} \Lambda_{2} \longrightarrow \cdots \stackrel{z^{\Delta_{t-1}}}{\longrightarrow} \Lambda_{t-1} \stackrel{z^{\Delta_{t}}}{\longrightarrow} M_{\Lambda} \stackrel{z^{v_{\Lambda}(M)}}{\longrightarrow} M .
$$

Let for simplicity $I=(1, \ldots, 1)$ and $\ell I=(\ell, \ldots, \ell)$.

Definition 18. For $D=\left(d_{1}, \ldots, d_{n}\right) \in \mathbb{Z}^{n}$, let $v=\min D$, let $t=\max D$ and

$$
D_{i}=\left(\min \left(d_{1}-v, i\right), \ldots, \min \left(d_{n}-v, i\right)\right) \text { for } 1 \leqslant i \leqslant t .
$$

The elementary splitting of $D$ is the unique decomposition $D=\Delta_{0}+\Delta_{1}+\cdots+\Delta_{t}$ where $\Delta_{0}=v I \in \mathbb{Z}^{n}$ and $\Delta_{k}=D_{k}-D_{k-1} \in\{0,1\}^{n}$ for $1 \leqslant k \leqslant t$.

We write $\Delta_{i}(D)$ if we need to specify the sequence $D$. The next straightforward lemma is left to the reader.

Lemma 19. Let $\mathbf{E D}_{\Lambda}(M)=\Delta_{0}+\Delta_{1}+\cdots+\Delta_{t}$ be the elementary splitting of $\mathbf{E D}_{\Lambda}(M)$ for $\Lambda, M \in \Lambda$. Then we have $\Delta_{0}=v_{\Lambda}(M) I$ and the following statements hold:

(i) $\delta_{i}=\operatorname{Tr} \Delta_{i}=\mu_{M}\left(\Lambda_{i}\right)$, where $\Lambda_{i} \in \Gamma_{\min }(\Lambda, M)$ as in (5).

(ii) $\Delta_{i}\left(\mathbf{E D}_{M}(\Lambda)\right)=I-\Delta_{d-i+1}\left(\mathbf{E D}_{\Lambda}(M)\right)$ for $1 \leqslant i \leqslant t$. In other terms,

$$
\mathbf{E D}_{M}(\Lambda)=\left(-t-v_{\Lambda}(M)\right) I+\left(I-\Delta_{t}\right)+\cdots+\left(I-\Delta_{1}\right)
$$

is the elementary splitting of $\mathbf{E D}_{M}(\Lambda)$.

(iii) Let $A=[\Lambda]$ and $B=[M]$. The gallery distance $\ell(A, B)$ is given by

$$
\ell(A, B)=\sum_{i=1}^{t-1}\left(n-\delta_{i}\right) \delta_{i+1} .
$$


Note that the sequence $\delta_{1}, \ldots, \delta_{t}$ is nonincreasing, and that we also have

$$
\delta_{i}=\left|\left\{1 \leqslant j \leqslant n \mid d_{j} \geqslant i+v_{\Lambda}(M)\right\}\right| .
$$

\section{Birkhoff-Grothendieck trivializations}

The central result in the theory of holomorphic vector bundles on $\mathbb{P}^{1}(\mathbb{C})$ is the Birkhoff-Grothendieck theorem, which states that any such bundle is isomorphic to a direct sum of line bundles. In this section, we take $X=\mathbb{P}^{1}(\mathbb{C})$, and we investigate what properties of the vector bundle can be retrieved by considering only the Bruhat-Tits building at a point $x \in X$.

3.1. The Birkhoff-Grothendieck property. According to Lemma 3, a holomorphic vector bundle $\mathscr{E} \in \mathscr{H}$ is completely described by the coset $\Lambda=[\mathscr{E}] \in \mathscr{H} / \sim_{x}$ and the lattice $\Lambda=\mathscr{E}_{x} \in \boldsymbol{\Lambda}$. Let us take up the notations of Section 2 again. Let $V$ denote the formalized meromorphic stalk $\widehat{\mathscr{V}_{x}}$ and let $B$ be the corresponding Bruhat-Tits building. Let $B_{0}=\left\{[M] \mid \mathscr{E}^{M}\right.$ is trivial $\}$ be the subset of trivializing lattices of $B$.

Note 20. Strictly speaking, $L \in B_{0}$ contains exactly one lattice $M \in L$ such that the extension $\mathscr{E}^{M}$ is trivial. Any other $\tilde{M} \in L$ gives a quasitrivial vector bundle $\mathscr{E} \tilde{\mathscr{M}}$. There is no real need to make the difference, since one gets a trivial bundle by simply tensoring with a line bundle.

Lemma 21. $B_{0} \neq \varnothing$ for any $x \in X$ and $\mathbf{\Lambda} \in \mathscr{H} / \sim_{x}$.

The existence of an analytic trivialization outside of an arbitrary point is a very general result on holomorphic bundles over complex analytic manifolds. This much more restrictive algebraicity statement follows easily from the BirkhoffGrothendieck theorem.

Proof. Let $\mathscr{E} \in \boldsymbol{\Lambda}$ be a representative of the coset $\boldsymbol{\Lambda}$. Let $X^{*}=X \backslash\{x\}$, and $\Delta$ a neighborhood of $x$. The Birkhoff-Grothendieck theorem implies that there exists a sequence of integers $\left(a_{1}, \ldots, a_{n}\right) \in \mathbb{Z}^{n}$, and linearly independent sections $\sigma_{1}, \ldots, \sigma_{n} \in \Gamma\left(X^{*}, \mathscr{E}\right)$ and $\widetilde{\sigma}_{1}, \ldots, \widetilde{\sigma}_{n} \in \Gamma(\Delta, \mathscr{\mathscr { C }})$ such that $\widetilde{\sigma}_{i}=z^{-a_{i}} \sigma_{i}$ for a local coordinate $z$ at $x$. The section $\sigma_{i}$ admits therefore a meromorphic extension at $x$, that we still denote $\sigma_{i}$. The trivial bundle $\mathscr{F} \in H_{0}$ spanned by $\left(\sigma_{1} \ldots, \sigma_{n}\right)$ is a trivialization of $\mathscr{E}$ at $x$ as required.

We say that any such bundle $\mathscr{F}$ is a Birkhoff-Grothendieck trivialization of $\mathscr{E}$ at $x$. We'll often write $\mathrm{BG}$ for short. Let $\mathrm{BG}_{x}(\mathscr{E}) \subset \mathscr{H}_{0}$ for the set of BG trivializations of $\mathscr{E}$ at $x$. Note that any $\mathscr{F} \in \mathrm{BG}_{x}(\mathscr{E})$ corresponds uniquely to a decomposition of $\mathscr{E}$ as a direct sum of line subbundles (a $B G$ decomposition)

$$
\mathscr{E}=\bigoplus_{i=1}^{n} \mathscr{L}_{i}
$$


Note 22. A line bundle on $P$ is characterized by its degree. Recall that, for any decomposition (6), if the integers $a_{i}=\operatorname{deg} \mathscr{L}_{i}$ satisfy $a_{1} \geqslant \cdots \geqslant a_{n}$, then the sequence $T(\mathscr{E})=\left(a_{1}, \ldots, a_{n}\right)$ is unique and called the type of $\mathscr{E}$.

In the case where the coset $[\mathscr{E}]=\Lambda \in \mathscr{H} / \sim_{x}$ is fixed, we write

$$
\operatorname{BG}(\Lambda)=\left\{M \in \Lambda^{0} \mid \mathscr{E}^{M} \in \mathrm{BG}_{x}(\mathscr{E})\right\},
$$

and $T(\Lambda)$ instead of $T(\mathscr{E})$. The global sections $\mathscr{F}(X)$ of a trivial bundle $\mathscr{F} \in[\mathscr{E}]$ induce, by taking stalks at $x$, a form $Y_{M}$ in the corresponding lattice $M=\mathscr{F}_{x} \in \boldsymbol{\Lambda}$, that we call the global form of $M$. The link between the Birkhoff-Grothendieck theorem and the algebraic structure of the local lattices is then given by the following straightforward characterization.

Lemma 23. The trivial lattice $M$ is in $\mathrm{BG}(\Lambda)$ if and only if there exists a Smith basis (e) of $M$ with respect to $\Lambda$ that is simultaneously a $\mathbb{C}$-basis of the global form $Y_{M}$ of $M$.

In this case, the basis (e) will be called a BG basis for $\Lambda$. We state now the following result separately for further reference.

Proposition 24. Let $\mathscr{E} \in \mathscr{H}$ be a holomorphic vector bundle. For any $B G$ trivialization $\mathscr{F}$ of $\mathscr{E}$ at any $x \in \mathbb{P}^{1}(\mathbb{C})$, the type $T(\mathscr{E})$ of the bundle $\mathscr{E}$ is equal to the sequence of elementary divisors $\mathbf{E D}_{\Lambda}(M)$ of the stalk $M=\mathscr{F}_{x}$ with respect to $\Lambda=\mathscr{E}_{x}$ (viewed as lattices in $\mathcal{V}_{x}$ ).

The BG trivializations of a bundle $\mathscr{E}$ are as a rule not unique, nor is any trivialization of $\mathscr{E}$ necessarily BG. One of the aims of this section is to prove the following local characterization of BG trivializations. Recall that the Bruhat-Tits building is endowed with three distance maps: the graph-theoretical distance $d$, the canonical metric $\boldsymbol{d}$ and the gallery distance $\ell$ (which is actually a pseudodistance on the vertices).

Theorem 25. Let $\Lambda=\mathscr{E}_{x}$. Let $\preceq_{\text {lex }}$ be the lexicographic, and $\preceq_{\text {lexrev }}$ the reverse lexicographic orderings on $\mathbb{Z}^{n}$. Then we have:

(i) $T(\mathscr{E}) \cong \min _{\preceq_{\text {lex }}}\left\{\mathbf{E D}_{\Lambda}(M)^{\searrow} \mid M \in \Lambda^{0}\right\}=\max _{\preceq_{\text {lexrev }}}\left\{\mathbf{E D}_{\Lambda}(M)^{\searrow} \mid M \in \Lambda^{0}\right\}$.

(ii) If $M \in \Lambda^{0}$ then the following conditions are equivalent:

(a) $M \in \mathrm{BG}(\Lambda)$;

(b) $\mathbf{E D}_{\Lambda}(M) \cong T(\mathscr{E})$;

(c) $\boldsymbol{d}(\Lambda, M)=\min _{\tilde{M} \in \mathbf{\Lambda}^{0}} \boldsymbol{d}(\Lambda, \tilde{M})$;

(d) $\ell(\Lambda, M)=\min _{\tilde{M} \in \Lambda^{0}} \ell(\Lambda, \tilde{M})$.

(iii) Moreover, if $M \in \operatorname{BG}(\Lambda)$ then $d(\Lambda, M)=\min _{\tilde{M} \in \Lambda^{0}} d(\Lambda, \tilde{M})$. 
In other terms, in the set of elementary divisors on $\Lambda^{0}$ with respect to $\mathscr{E}_{x}$, ordered by decreasing values, the minimum by direct lexicographic ordering coincides with the maximum by reverse lexicographic ordering, and this value is exactly the type $T(\mathscr{E})$. Moreover, any trivial lattice having $T(\mathscr{E})$ as elementary divisors is a BG trivialization of $\mathscr{E}$. Finally, the BG trivializations of a lattice $\Lambda$ are exactly the geodesic projections of $\Lambda$ onto $\Lambda^{0}$ for both metrics $\boldsymbol{d}$ and $\ell$. This result will be proved in two steps: Section 3.2 is dedicated to proving the first item and the implications (a) $\Rightarrow$ (b), (c), (d), (iii), and Section 3.3 to the converse implications.

3.1.1. Monopoles and $B G$ trivializations. Recall from Section 1 that the group of monopole gauge transforms at $x$ sends a trivial sheaf $\mathscr{F}_{\mathscr{F}}$ to a trivial sheaf $\widetilde{F}_{\mathscr{F}}$ such that $\mathscr{F}_{\mid X \backslash\{x\}}=\widetilde{\mathscr{F}}_{\mid X \backslash\{x\}}$. This group is described by the group of unimodular polynomial matrices $\mathrm{GL}_{n}(\mathbb{C}[T])$, that is, matrices of the form

$$
P=P_{0}+P_{1} T+\cdots+P_{k} T^{k} \quad \text { where } \exists \alpha \in \mathbb{C}^{*} \text {, det } P=\alpha .
$$

More precisely, assuming that $x=0$, a matrix $\Pi$ is a monopole at $x$ if and only $\Pi=P(1 / z)$ with $P \in \mathrm{GL}_{n}(\mathbb{C}[X])$ and $z$ is the standard coordinate on $\mathbb{P}^{1}(\mathbb{C})$.

A trivial bundle $\mathscr{F} \in \mathscr{H}_{0}$ is a trivialization of $\mathscr{E} \in \mathscr{H}$ at $x$ if any basis $(\sigma)=$ $\left(\sigma_{1}, \ldots, \sigma_{n}\right)$ of global sections of $\mathscr{F}$ spans the stalk $\mathscr{E} y$ over the local ring $\mathscr{O}_{y}=\left(\mathscr{O}_{X}\right)_{y}$ for every $y \neq x$. Any other basis of meromorphic sections $(\widetilde{\sigma})$ spans a trivialization $\widetilde{F}$ of $\mathscr{E}$ at $x$ if and only if the gauge $\Pi$ from $(\sigma)$ to $(\widetilde{\sigma})$ is a monopole at $x$. In particular, since the group of units of $\mathbb{C}[X]$ is $\mathbb{C}^{*}$, a line bundle $\mathscr{L}$ admits a unique trivialization $\mathscr{L}^{x}$ at $x$.

Lemma 26. Two $B G$ bases for $\Lambda$ are related by $a-T(\Lambda)$-parabolic monopole gauge.

Proof. Consider two BG trivializations $M, \tilde{M}$ of $\Lambda$, as in the following diagram, where $D=T(\Lambda)$ :

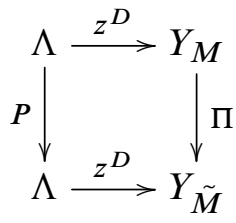

Since $v\left(P_{i j}\right) \geqslant 0$, the gauge $\Pi$ satisfies $v\left(\Pi_{i j}\right) \geqslant d_{j}-d_{i}$. Therefore $\Pi_{i j}=0$ as soon as $d_{j}>d_{i}$, which means that $\Pi$ is $(-D)$-parabolic.

According to the previous section, $\mathscr{F}$ is a BG trivialization of $\mathscr{E}$ at $x$ if there exists a basis $(\sigma)=\left(\sigma_{1}, \ldots, \sigma_{n}\right)$ of global sections of $\mathscr{F}$ and an integer sequence $D=\left(d_{1}, \ldots, d_{n}\right)$, such that $(e)=\left(t^{-d_{1}} \sigma_{1}, \ldots, t^{-d_{n}} \sigma_{n}\right)$ spans the stalk $\mathscr{E}_{x}$ over the local ring $\mathrm{O}=\left(\mathrm{O}_{X}\right)_{x}$, where $t$ is a local coordinate at $x$. This coordinate $t$ can be arbitrarily chosen, since the local behavior of $\mathscr{E}$ only depends on the local ring $\mathcal{O}$. If we choose as coordinate $t$ a meromorphic function on $X$, then the sections 
(e) $=\left(t^{-d_{1}} \sigma_{1}, \ldots, t^{-d_{n}} \sigma_{n}\right)$ form a basis of global (meromorphic) sections of $\mathscr{V}$. The $\mathbb{O}_{X}$-module $\widetilde{\mathscr{F}}$ spanned by $(e)$ in this case does coincide with $\mathscr{E}$ at $x$, and differs from it at most on the support of the divisor of the function $t$.

When $X=\mathbb{P}^{1}(\mathbb{C})$, we can obviously find a function $t$ with divisor $(t)=x-y$ for any arbitrary point $y \neq x$, that we call a global coordinate at $x$. In this case, the bundle $\widetilde{F}$ is a BG trivialization of $\mathscr{E}$ at $y$. It is clearly independent of the global basis $(\sigma)$ of $\mathscr{F}$, which is defined up to a $(-D)$-parabolic constant matrix $C \in \mathrm{GL}_{n}(\mathbb{C})$, and of the specific meromorphic function $t$, which is only defined up to a nonzero constant. We call $t_{y}(\mathscr{F})=\widetilde{\mathscr{F}}$ the transport at $y$ of the BG trivialization $\mathscr{F}_{\mathscr{F}}$ of $\mathscr{E}$ at $x$.

Understood otherwise, this is the description of a nontrivial bundle $\mathscr{E}$ by means of two trivial bundles $\mathscr{F}$ and $\widetilde{\mathscr{F}}$ coinciding outside $\{x, y\}$, and glued along the cocycle $g=t^{D}$, where $(t)=x-y$.

3.1.2. The Harder-Narasimhan flag. The Harder-Narasimhan filtration $\mathrm{HN}(\mathscr{E})$ of $\mathscr{E}$ over $\mathbb{P}^{1}(\mathbb{C})$ can be obtained (see [Sabbah 2002, p. 65]) from a BG decomposition $\mathscr{E}=\bigoplus_{i=1}^{n} \mathscr{L}_{i}$ of $\mathscr{E}$ as a direct sum of line bundles $\mathscr{L}_{i} \simeq \mathcal{O}\left(a_{i}\right)$ of the appropriate degree, by

$$
F^{k}(\mathscr{E})=\bigoplus_{i \mid a_{i} \geqslant k} \mathscr{L}_{i}
$$

Locally, the Harder-Narasimhan filtration can be described as follows. Let $(e)$ be a BG basis for $\Lambda=\mathscr{E}_{x}$. The Harder-Narasimhan flag $\mathrm{HN}_{\Lambda}$ of $V=\mathscr{V}_{x}$ defined by

$$
F^{k}=\bigoplus_{i \mid a_{i} \geqslant k} K e_{i}
$$

is independent of the BG basis for $\Lambda$. For a lattice $M$, define the residual HN flag

$$
\boldsymbol{H}_{\Lambda}^{M}=\left(\mathrm{HN}_{\Lambda} \cap M\right) / \mathfrak{m} M \quad \text { and, for simplicity, } \quad \boldsymbol{H}^{\Lambda}=\boldsymbol{H}_{\Lambda}^{\Lambda} .
$$

Lemma 27. Let $\Lambda \in \Lambda$ and let $\mathrm{HN}_{\Lambda}$ be the HN flag of $V$.

(i) If $M \in \mathrm{BG}(\Lambda)$, then $\boldsymbol{H}_{\Lambda}^{M}=\boldsymbol{F}^{M}(\Lambda)$, hence $\boldsymbol{H}^{\Lambda}$ is transversal to $\boldsymbol{F}^{\Lambda}(M)$.

(ii) Conversely, for any flag $\boldsymbol{F}^{\prime}$ which is transversal to $\boldsymbol{H}^{\Lambda}$, there exists $M \in \mathrm{BG}(\Lambda)$ such that $\boldsymbol{F}^{\Lambda}(M)=\boldsymbol{F}^{\prime}$.

Proof. Let $T=T(\Lambda)=\operatorname{diag}\left(a_{1} I_{n_{1}}, \ldots, a_{s} I_{n_{s}}\right)$ with $a_{i}>a_{i+1}$ be the type of the lattice $\Lambda$, so that

$$
\Lambda \stackrel{z^{T}}{\longrightarrow} M
$$

sends the basis $(e)$ of $\Lambda$ into the global basis $(\varepsilon)=z^{T}(e)$ of $Y_{M}$. Let $v_{i}=$ $\sum_{1 \leqslant k \leqslant i} n_{i}$, and let generically $\bar{x}$ denote the class modulo $\mathfrak{m}$ of a vector $x$. The $i$-th component of the flag $\boldsymbol{H}_{\Lambda}^{M}$ is spanned by $\left(\bar{\varepsilon}_{1}, \ldots, \bar{\varepsilon}_{\boldsymbol{v}_{i}}\right)$, so $\boldsymbol{H}_{\Lambda}^{\boldsymbol{M}}=\boldsymbol{F}^{\boldsymbol{M}}(\Lambda)$ indeed 
holds. Conversely, the $(n-i+1)$-th component of $\boldsymbol{F}^{\Lambda}(M)$ is $F_{i}=\left\langle\bar{e}_{\nu_{i}+1}, \ldots, \bar{e}_{n}\right\rangle$ while the $i$-th component $H_{i}$ of $\boldsymbol{H}^{\Lambda}$ satisfies $H_{i}=\left\langle\bar{e}_{1}, \cdots, \bar{e}_{v_{i}}\right\rangle$; hence both flags are transversal to each other. Any other BG trivialization $\tilde{M}$ is obtained from $(\varepsilon)$ by a monopole gauge transform $\Pi$ such that $P=z^{T} \Pi z^{-T} \in \mathrm{GL}_{n}(\mathrm{O})$. According to Lemma 26, $\Pi$ is block-upper-triangular with respect to the blocks of equal elements of $T$, hence so is $P$. Let $P \in G_{T}(\mathbb{C})$. If $z$ is a global coordinate, the matrix $z^{-T} P z^{T}$ is a monopole. The orbit of $(\varepsilon)$ under the set of the constant $T$-parabolic matrices covers the set of all flags in $E$ which are transversal to the image of $\mathrm{HN}(\mathscr{E})_{x}$ in $E$.

For any BG trivialization $\mathscr{F}$ of $\mathscr{E}$ at $x$, let $Y=\Gamma(X, \mathscr{F})$ be the $\mathbb{C}$-vector space of global sections of $\mathscr{F}$. The Harder-Narasimhan filtration $\mathrm{HN}(\mathscr{E})$ also induces a canonical filtration $\mathrm{HN}_{\Lambda}(Y)$ of $\mathbb{C}$-vector spaces of $Y$. To avoid defining new concepts, we will also refer to this filtration as the Harder-Narasimhan filtration of $Y$. Note that it depends solely on the lattice $\Lambda \in \boldsymbol{\Lambda}$.

3.2. Modification of the type. We wish to answer algebraically the following question: "what does the type of $\mathscr{E}$ become when the stalk $\mathscr{E}_{x}=\Lambda$ at $x$ is replaced by another lattice $\tilde{\Lambda}$ ?" It turns out that the question can be very explicitly answered when the lattice $\tilde{\Lambda}$ is not too far from $\Lambda$, namely at distance 1 in the graph-theoretic distance of the Bruhat-Tits building. The following proposition generalizes a result of Gabber and Sabbah.

Proposition 28. Let $\mathscr{E} \simeq \bigoplus_{i=1}^{n} \mathcal{O}\left(a_{i}\right)$ be a holomorphic vector bundle on $X=$ $\mathbb{P}^{1}(\mathbb{C})$, with $a_{1} \geqslant \cdots \geqslant a_{n}$, and let $x \in X$. Let $\tilde{\Lambda} \in \Lambda_{x}$ be a lattice such that $\mathfrak{m}_{x} \mathscr{E}_{x} \subset \tilde{\Lambda} \subset \mathscr{E}_{x}$. Let $E=\mathscr{E}_{x} / \mathfrak{m}_{x} \mathscr{E}_{x}$ be the local fiber at $x$, let

$$
\boldsymbol{H}: H_{0}=0 \subset H_{1} \subset \cdots \subset H_{S}=E
$$

be the residual $H N$ flag in $E$, and $W=\tilde{\Lambda} / \mathfrak{m}_{x} \mathscr{E}_{x}$ the image of $\tilde{\Lambda}$. Assume that the type of $\mathscr{E}$ is written as

$$
a=(\underbrace{a_{1}, \ldots, a_{1}}_{n_{1} \text { times }}, \underbrace{a_{2}, \ldots, a_{2}}_{n_{2} \text { times }}, \ldots, \underbrace{a_{s}, \ldots, a_{s}}_{n_{s} \text { times }}) .
$$

Then the modified bundle $\mathscr{F}=\mathscr{E}^{\tilde{\Lambda}}$ has type

$$
\tilde{a}=(\underbrace{a_{1}, \ldots, a_{1}}_{m_{1} \text { times }}, \underbrace{a_{1}-1, \ldots, a_{1}-1}_{n_{1}-m_{1} \text { times }}, \ldots, \underbrace{a_{s}, \ldots, a_{s}}_{m_{s} \text { times }}, \underbrace{a_{s}-1, \ldots, a_{s}-1}_{n_{s}-m_{s} \text { times }})
$$

where $m_{i}=\operatorname{dim}_{\mathbb{C}} H_{i} \cap W-\operatorname{dim}_{\mathbb{C}} H_{i-1} \cap W$.

Proof. This is explained in the following scheme. Let $\Lambda=\mathscr{E}_{x}$, and let $t$ be a local coordinate at $x$ (that we assume without loss of generality to be $\infty$ ). Let 
$D=\operatorname{diag}\left(a_{1}, \ldots, a_{n}\right)$ be the elementary divisors of the BG trivialization $M$ in $\Lambda$ (or, in this case, the type of $\mathscr{E}$ ).

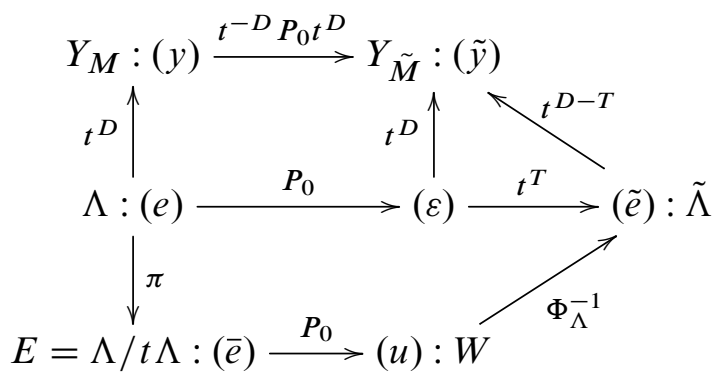

Let $(e)$ be a basis of $\Lambda$, such that $(\sigma)=\left(t^{D} e\right)$ is a basis of the form $Y_{M}$. Under the canonical projection $\pi: \Lambda \rightarrow E=\Lambda / t \Lambda$, the HN filtration of $\Lambda$ descends to a flag of $\mathbb{C}$-vector spaces $\boldsymbol{H}: 0=H_{0} \subset \cdots \subset H_{s}=E$, and the quotient basis $(\bar{e})$ is a basis respecting this flag. Let $t \Lambda \subset \widetilde{\Lambda} \subset \Lambda$ be the new lattice, and let $W \subset E$ be the subspace it is projected upon by $\pi$. Let $(u)$ be a basis respecting both $W$ and the flag $\boldsymbol{H}$, and let $P_{0}$ be a change of basis from (e) to (u). Consequently, the matrix $P_{0}$ belongs to the parabolic subgroup $P_{\boldsymbol{H}}$ stabilizing the flag $\boldsymbol{H}$; therefore it is block-upper-triangular, with blocks given by the equal elements among the $a_{i}$. Define now the basis $(\varepsilon)$ of $\Lambda$ as the image of $(e)$ under the constant gauge $P_{0}$. Here is where $d(\Lambda, \widetilde{\Lambda}) \leqslant 1$ is important: the basis $(\varepsilon)$ is a Smith basis of $\tilde{\Lambda}$ (this would be not necessarily true if the lattices were further apart). Let $T=\operatorname{diag}\left(t_{1}, \ldots, t_{n}\right)$ be the diagonal matrix such that $t_{i}=0$ if $\pi\left(\varepsilon_{i}\right) \in W$ and $t_{i}=1$ otherwise. Then $(\tilde{e})=t^{T}(\varepsilon)$ is a basis of $\widetilde{\Lambda}$. Let now $(\widetilde{\varepsilon})=t^{D}(\varepsilon)$ be the basis of $\tilde{M}$ deduced from $(\varepsilon)$. The matrix of the basis change from $\tilde{\Lambda}$ to $\tilde{M}$ corresponding to the bases $(\sigma)$ and $(\widetilde{\varepsilon})$ is equal to $Q=t^{-D} P_{0} t^{D}=\left(P_{0}\right)_{i j} t^{d_{j}-d_{i}}$. Now, since $P_{0} \in P_{\boldsymbol{H}}$, we have $\left(P_{0}\right)_{i j}=0$ whenever $d_{i}-d_{j}<0$. Therefore this gauge $Q=\frac{1}{t^{k}} Q_{k}+\cdots+Q_{0}$ is a Laurent polynomial in $t$ with only nonpositive terms, where moreover $Q_{0} \in \mathrm{GL}_{n}(\mathbb{C})$. Since $X=\mathbb{P}^{1}(\mathbb{C})$, it is possible to choose as local coordinate at $\infty$ a meromorphic function with divisor $(\infty)-(0)$, namely $t=1 / z$. Accordingly, $Q$ is a polynomial in $z$, whereas $\operatorname{det} Q=\operatorname{det} P_{0} \in \mathbb{C}^{*}$. Hence $Q \in \mathrm{GL}_{n}(\mathbb{C}[z])$ is a monopole gauge. Since $(\sigma)$ was a basis of global meromorphic sections of $E$, then $(\widetilde{\mathcal{E}})$ also is. Therefore $\tilde{M} \in B_{0}$ is a trivializing lattice. Moreover, $\tilde{M}$ is a BG trivialization of both $\mathscr{E}$ and $\mathscr{F}=\mathscr{E} \tilde{\Lambda}$, because the basis $(\widetilde{\varepsilon})$ is a Smith basis for $\Lambda$ and $\tilde{\Lambda}$. Therefore, we can explicitly compute the new elementary divisors of $\tilde{\Lambda}$ in $\tilde{M}$, which are given by the matrix $D-T$. Summing up, we see that the change of lattice has subtracted 1 from all the elementary divisors corresponding to the vectors of the basis $(\varepsilon)$ whose image under $\pi$ do not fall into the subspace $W$. We obtain the Harder-Narasimhan filtration of the modified bundle by reordering the type by decreasing values. 
Proposition 28 generalizes the construction given by Sabbah based on an idea of O. Gabber in [Sabbah 2002, Proposition 4.11] (where only the case where $W$ is one-dimensional is tackled). This result is independent of the valuation of $\tilde{\Lambda}$, and can thus be formulated as follows.

Corollary 29. Let $\Lambda, \widetilde{\Lambda} \in \boldsymbol{\Lambda}$, and let $\boldsymbol{H}=\left(\mathrm{HN}_{\Lambda} \cap \Lambda\right) / \mathfrak{m} \Lambda$ be the residual $H N$ flag and $\boldsymbol{F}=\boldsymbol{F}^{\Lambda}(\tilde{\Lambda})=(0 \subset W \subset \Lambda / \mathfrak{m} \Lambda)$ the flag induced by $\tilde{\Lambda}$ in $\Lambda / \mathfrak{m} \Lambda$. If $d(\Lambda, \tilde{\Lambda})=1$, then we have

$$
T(\tilde{\Lambda}) \cong T(\Lambda)^{\searrow}-\sigma\left(\mathbf{E D}_{\Lambda}(\tilde{\Lambda})^{\nearrow}\right)
$$

where $\sigma \in \rho(\boldsymbol{F}, \boldsymbol{H})$ represents the relative position of $\boldsymbol{F}$ and $\boldsymbol{H}$. Actually, one can even say, putting $\widetilde{\boldsymbol{H}}=\left(\mathrm{HN}_{\tilde{\Lambda}} \cap \Lambda\right) / \mathfrak{m} \Lambda$, that

$$
T(\tilde{\Lambda})^{\searrow}=\rho(\boldsymbol{H}, \tilde{\boldsymbol{H}}) T(\Lambda)^{\searrow}-\rho(\boldsymbol{F}, \tilde{\boldsymbol{H}})\left(\mathbf{E D}_{\Lambda}(\tilde{\Lambda})^{\nearrow}\right) .
$$

Proof. With the notations of the diagram (8), the basis $(\tilde{y})$ is a common BG basis for $\Lambda$ and $\tilde{\Lambda}$. Therefore, the HN flags are spanned in $(\tilde{y})$ over $K$ by the flags of indices $S^{\searrow}(D)$ and $S^{\searrow}(D-T)$, respectively. By applying any representative of the coset $\rho(\boldsymbol{H}, \tilde{\boldsymbol{H}})$ to $D-T$, one gets $T(\tilde{\Lambda})^{\searrow}$. Therefore, $T(\widetilde{\Lambda})^{\searrow}=\rho(\boldsymbol{H}, \tilde{\boldsymbol{H}}) T(\Lambda)^{\searrow}-$ $\rho(\boldsymbol{H}, \tilde{\boldsymbol{H}}) \rho(\boldsymbol{F}, \boldsymbol{H})\left(\mathbf{E D}_{\Lambda}(\widetilde{\Lambda})^{\nearrow}\right)$. Take as representatives of the cosets $\rho(\boldsymbol{H}, \tilde{\boldsymbol{H}})$ and $\rho(\boldsymbol{F}, \boldsymbol{H})$ their minimal length element (see, e.g., [Abramenko and Brown 2008, Proposition 2.23, p. 83]). Since the quotient basis $(\bar{\varepsilon})$ of $\Lambda / \mathfrak{m} \Lambda$ is adapted to the three flags $\boldsymbol{H}, \widetilde{\boldsymbol{H}}$ and $\boldsymbol{F}$, we get $\rho(\boldsymbol{H}, \tilde{\boldsymbol{H}}) \rho(\boldsymbol{F}, \boldsymbol{H})=\rho(\boldsymbol{F}, \tilde{\boldsymbol{H}})$ [Abramenko and Brown 2008, Lemma 5.55, p. 236].

Corollary 30. Let $\Lambda \in \Lambda$. Then we have

(i) $\operatorname{BG}(\Lambda) \neq \varnothing$,

(ii) for any adjacent lattice $\tilde{\Lambda}$, we have $\mathrm{BG}(\Lambda) \cap \mathrm{BG}(\tilde{\Lambda}) \neq \varnothing$,

(iii) all the elements in a chamber of $B_{n}$ have a common $B G$ trivialization.

Proof. According to Proposition 28, if a lattice $\Lambda$ admits a BG trivialization, so does $M$ for any adjacent lattice $M$. However, according to Proposition 17, two lattices are always connected by a path of adjacent lattices. Since a trivial lattice is its own BG trivialization, the two first results are simultaneously established. The third stems from the fact that any chamber appears as a complete flag in the quotient space of any representative. According to Bruhat's lemma, there is a basis respecting simultaneously two flags, in this case the one corresponding to the chamber and the one induced by the HN filtration.

3.2.1. An algorithm to compute a Birkhoff-Grothendieck trivialization. Let $x \in X$, and let $\boldsymbol{\Lambda}=[\mathscr{E}]_{x}$ be the $\sim_{x}$-equivalence class of $\mathscr{E}$. Let $\Lambda=\mathscr{E}_{x}$ and $M=\mathscr{F}_{x} \in \boldsymbol{\Lambda}$ where $\mathscr{F}$ is an arbitrary trivialization of $\mathscr{E}$ at $x$. In this local setting, we "see" the 
global sections of $\mathscr{F}$ as the global form $Y \subset M$. An arbitrary trivialization of $\mathscr{E}$ is not necessarily BG. The following result is easily established.

Lemma 31. Let $M \in \mathrm{BG}(\Lambda)$. Then we have

(i) $M \in \mathrm{BG}\left(z^{k} \Lambda\right)$ for $k \in \mathbb{Z}$,

(ii) $M \in \operatorname{BG}\left(\Lambda^{\prime}\right)$ for any lattice $\Lambda^{\prime}$ on the shortest path $\Gamma_{\min }(\Lambda, M)$.

Proposition 28 allows to construct effectively from an arbitrary trivialization $M$ a BG one, by following shortest paths in the Bruhat-Tits building from $M$ to $\mathscr{E}_{x}$. The following result shows how to start the construction. We can assume that $v_{M}(\Lambda)=0$.

Lemma 32. Let $M \in \Lambda^{0}$ be a trivial lattice in $B_{n}$. For any lattice $\Lambda \in \Lambda$ such that $d(\Lambda, M)=1$, we have $M \in \mathrm{BG}(\Lambda)$. More precisely, let $Y \subset M$ be the global form of $M$. For any basis (e) respecting $W=\Lambda / \mathfrak{m} M$, the $Y$-basis $\left(e_{Y}\right)$ is a Smith basis for $\Lambda$.

Proof. Assume that (e) satisfies the assumptions of the lemma. Then, according to Lemma 13, the $Y$-basis $\left(e_{Y}\right)$ is a Smith basis of $M$ for $\Lambda$. Since it is a basis of the global form of $M$, the result follows, and in particular, the HarderNarasimhan filtration of the corresponding bundle is equal to the $Y$-lifting of the flag $(0 \subset W \subset M / \mathfrak{m} M)$.

If there existed a Smith basis of $M$ for $\Lambda$ which spanned simultaneously $Y_{M}$, the lattice $M$ would be a BG trivialization of $\Lambda$, and the sequence $\mathbf{E D}_{\Lambda}(M)$ would represent the type of $\mathscr{E}$.

Theorem 33. Let $\Lambda \in \boldsymbol{\Lambda}=[\mathscr{E}]$, and let $M \in \mathbf{\Lambda}^{0}$ be an arbitrary trivial lattice. Let $\mathbf{E D}_{\Lambda}(M)=\Delta_{0}+\cdots+\Delta_{t}$ be the elementary splitting of $\mathbf{E D}_{\Lambda}(M)$. There exists a sequence of permutations $w_{k} \in S_{n}$ such that the type $T(\Lambda)$ satisfies

$$
T(\Lambda) \cong \Delta_{0}+w_{1} \Delta_{1}+w_{2} \Delta_{2}+\cdots+w_{t} \Delta_{t} .
$$

Proof. We prove the result first on the sequence $D=\mathbf{E D}_{M}(\Lambda)=-\mathbf{E D}_{\Lambda}(M)$. Let $\Gamma=\left(M=M_{0}, M_{1}, \ldots, M_{t}=\Lambda_{M}\right)$ be (a normalized representative of $)$ the min-shortest path from $[M]$ to $[\Lambda]$. Let $D=\mathbf{E D}_{M}\left(\Lambda_{M}\right)=\left(\boldsymbol{k}_{1} I_{n_{1}}, \ldots, \boldsymbol{k}_{s} I_{n_{s}}\right)$ where $0=\boldsymbol{k}_{1}<\cdots<\boldsymbol{k}_{s}=t$. Consider the elementary splitting of $D$

$$
D=\Delta_{1}+\cdots+\Delta_{t} \quad \text { where } \Delta_{i}=\left(0_{n-m_{i}}, I_{m_{i}}\right)
$$

for a nonincreasing sequence $\left(m_{i}\right)$. Recall that

$$
D_{k}=\Delta_{1}+\cdots+\Delta_{k}=\mathbf{E D}_{M}\left(M_{k}\right) .
$$

Let $(e)$ be a Smith basis of $M$ for $\Lambda$, and let $\left(e^{(k)}\right)=z^{D_{k}}(e)$ for $0 \leqslant k \leqslant t$ (with $D_{0}=0$ by convention). The induced basis $\left(\bar{e}^{(k)}\right)$ of $E_{k}=M_{k} / \mathfrak{m} M_{k}$ respects both flags $\boldsymbol{F}^{M_{k}}(M)$ and $\boldsymbol{F}^{M_{k}}(\Lambda)$. With the help of Proposition 28, 
we construct a BG trivialization $\tilde{M}_{k+1}$ of the $k$-th element $M_{k}$ of the shortest path $\Gamma$, which is simultaneously a BG trivialization of $\Lambda_{M}+\mathfrak{m} M_{k}=\Lambda_{M}+$ $\mathfrak{m}\left(\Lambda_{M}+\mathfrak{m}^{k} M\right)=M_{k+1}$.

Let us describe this in more detail. By assumption, there exists a BG basis $(\sigma)$ of $\tilde{M}_{k}$ for $M_{k}$. Let

$$
T=\mathbf{E D}_{\tilde{M}_{k}}\left(M_{k}\right)^{\nearrow} \quad \text { and } \quad T^{\prime}=\mathbf{E D}_{M_{k}}\left(\Lambda_{M}\right)^{\nearrow} .
$$

Let $(y)=z^{T}(\sigma)$ be the corresponding basis of $M_{k}$, and $(\varepsilon)$ a Smith basis of $M_{k}$ for $\Lambda_{M}$. The gauge $U$ from $(y)$ to $(\varepsilon)$ can be factored as

$$
U=U_{0}\left(I+t U^{\prime}\right) \quad \text { with } U_{0} \in \mathrm{GL}_{n}(\mathbb{C}) .
$$

Since $(\sigma)$ is a basis of the global form $Y_{\tilde{M}_{k}}$, the basis $(\bar{y})$ of $E=M_{k} / \mathfrak{m} M_{k}$ is strictly adapted to the HN flag

$$
\boldsymbol{H}^{(k)}=\left(\mathrm{HN}_{M_{k}} \cap M_{k}\right) / \mathfrak{m} M_{k} .
$$

Similarly, $(\bar{\varepsilon})$ is strictly adapted to the flag $\boldsymbol{F}^{(k)}=\boldsymbol{F}^{M_{k}}\left(\Lambda_{M}\right)$. Let $B$ be the standard Borel subgroup of $\mathrm{GL}_{n}(\mathbb{C})$. By the Bruhat decomposition, the group $\mathrm{GL}_{n}(\mathbb{C})$ is a disjoint union of double cosets:

$$
\mathrm{GL}_{n}(\mathbb{C})=\coprod_{w \in W} B w B
$$

where $W$ is the Weyl group $W=S_{n}$. The constant term $U_{0}$ of the gauge $U$ belongs to only one such cell: let $w \in S_{n}$ be the label of the corresponding Schubert cell. We have a decomposition $U_{0}=Q P_{w} Q^{-1}$ with $Q, Q^{\prime} \in B$, where $P_{w}$ is the matrix representation of the permutation $w$. Accordingly, the gauge transforms $Q$ and $Q^{\prime}$ respect, respectively, the flags $\boldsymbol{H}=\boldsymbol{H}^{(k)}$ and $\boldsymbol{F}=\boldsymbol{F}^{(k)}$. In the quotient space $E$, we have:

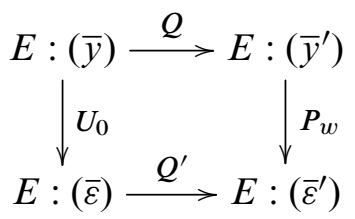

The gauge $U_{0}$ represents geometrically the change of a basis that spans the HarderNarasimhan flag $\boldsymbol{H}$ to one that spans the flag $\boldsymbol{F}$ induced by $\Lambda$; therefore $w$ is a representative of the relative position $\rho(\boldsymbol{H}, \boldsymbol{F})$.

Let $T^{\prime}=\Delta_{k+1}+\cdots+\Delta_{t}$ be the elementary splitting of $T^{\prime}$. Since $(\bar{\varepsilon})$ respects the flag $\boldsymbol{F}$, it will in particular respect the trace of the first element $M_{k+1}=\Lambda+\mathfrak{m} M_{k}$ of the shortest path $\Gamma_{\min }\left(M_{k}, \Lambda\right)$; therefore any lifting of $(\bar{\varepsilon})$ will be a Smith basis of $M_{k+1}$ with elementary divisors $\Delta_{k+1}$. Put $T^{\prime \prime}=T^{\prime}-\Delta_{k+1}$. The previous 
scheme gets thus lifted to the following complete picture.

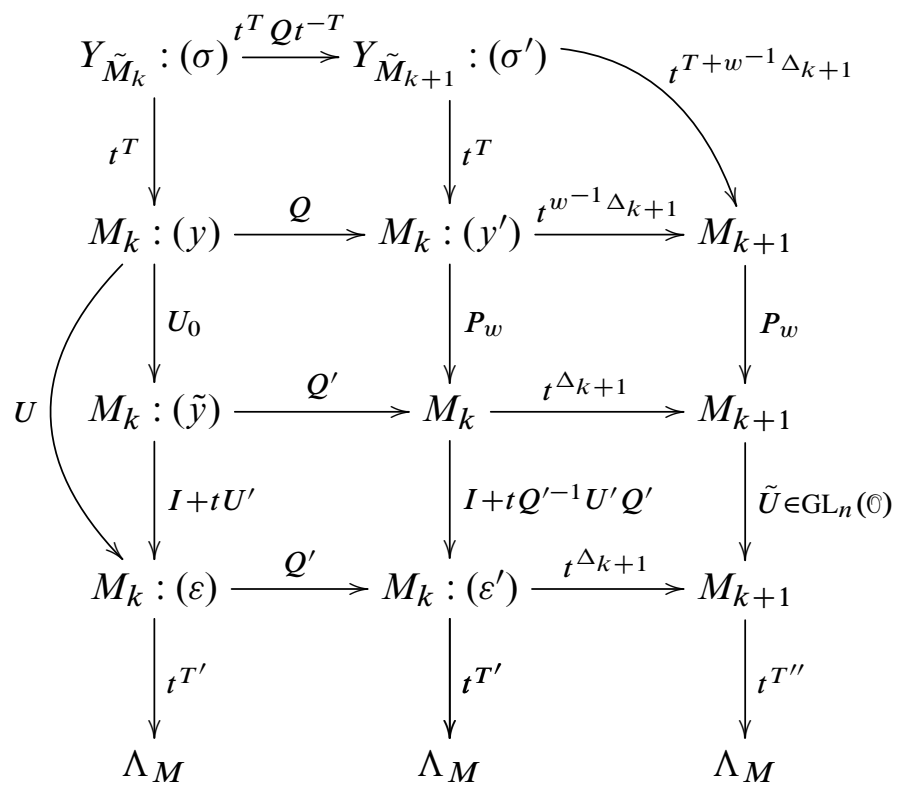

As a result, the elementary divisors of $M_{k+1}$ with respect to the common BG trivialization $\tilde{M}_{k+1}$ of $M_{k}$ and $M_{k+1}$ are not $T+\Delta_{k+1}$ (as with respect to $\tilde{M}_{k}$ ), but $T+w^{-1} \Delta_{k+1}$; namely, the elements of $\Delta_{k+1}$ have been twisted according to the permutation $w^{-1}=\rho(\boldsymbol{F}, \boldsymbol{H})$ indexing the Bruhat cell that contains the matrix $U_{0} \in \mathrm{GL}_{n}(\mathbb{C})$. The resulting matrix $\tilde{T}=T+w^{-1} \Delta_{k+1}$ is not necessarily ordered by increasing values: therefore we cannot ensure that $w_{k+1}=\rho(\boldsymbol{F}, \boldsymbol{H})$, since the ordered diagonal has the form $\sigma T+\sigma w^{-1} \Delta_{k+1}$. According to Corollary 29, however, we know that we can take

$$
\sigma=\rho\left(\boldsymbol{H}^{\boldsymbol{M}_{k}}, \boldsymbol{H}_{\boldsymbol{M}_{\boldsymbol{k}}}^{\boldsymbol{M}_{k+1}}\right)=\rho\left(\mathrm{HN}_{\boldsymbol{M}_{k}}, \mathrm{HN}_{\boldsymbol{M}_{k+1}}\right) .
$$

Thus

$$
\tilde{T}^{\nearrow}=\rho\left(\mathrm{HN}_{\boldsymbol{M}_{k}}, \mathrm{HN}_{\boldsymbol{M}_{k+1}}\right) T+\rho\left(\boldsymbol{H}^{\boldsymbol{M}_{k}}, \boldsymbol{H}_{\boldsymbol{M}_{k}}^{\boldsymbol{M}_{k+1}}\right) \rho\left(\boldsymbol{F}^{(k)}, \boldsymbol{H}^{\boldsymbol{M}_{k}}\right) \Delta_{k+1} .
$$

Putting $T_{k}=\mathbf{E D}_{\tilde{M}_{k}}\left(M_{k}\right)^{\nearrow}$, we get

$$
T_{k+1}=\rho\left(\mathrm{HN}_{\boldsymbol{M}_{\boldsymbol{k}}}, \mathrm{HN}_{\boldsymbol{M}_{k+1}}\right) T_{k}+\rho\left(\boldsymbol{F}^{\boldsymbol{M}_{k}}(\Lambda), \boldsymbol{H}_{\boldsymbol{M}_{k}}^{\boldsymbol{M}_{k+1}}\right) \Delta_{k+1} .
$$

At the end of at most $t$ steps, the lattice $\tilde{M}_{t}$ is a BG trivialization of $\Lambda_{M}$, and thus of $\Lambda$. We have $\tilde{T}=w_{1} \Delta_{1}+w_{2} \Delta_{2}+\cdots+w_{t} \Delta_{t}$ such that

$$
\tilde{M}_{t} \stackrel{z^{\widetilde{T}}}{\longrightarrow} \Lambda M \stackrel{z^{v_{M}(\Lambda)}}{\longrightarrow} \Lambda .
$$


Since $T(\Lambda)=\mathbf{E D}_{\Lambda}\left(\tilde{M}_{t}\right)=-\tilde{T}-v_{M}(\Lambda)$, we get

$$
\begin{aligned}
T(\Lambda) & =-\Delta_{0}-w_{1} \Delta_{1}-w_{2} \Delta_{2}-\cdots-w_{t} \Delta_{t} \\
& =-\Delta_{0}-t I+w_{1}\left(I-\Delta_{1}\right)+\left(I-w_{2} \Delta_{2}\right)+\cdots+\left(I-w_{t} \Delta_{t}\right) \\
& =\left(-\Delta_{0}-t I\right)+w_{t}\left(I-\Delta_{t}\right)+w_{t-1}\left(I-\Delta_{t-1}\right)+\cdots+w_{1}\left(I-\Delta_{1}\right) .
\end{aligned}
$$

By Lemma 19, the result is established. Note that a similar relation holds for $\mathbf{E D}_{M}(\Lambda)$, and that if $T(\Lambda)=\Delta_{0}+w_{1} \Delta_{1}+w_{2} \Delta_{2}+\cdots+w_{t} \Delta_{t}$, then $T\left(\Lambda_{k}\right)=$ $\Delta_{0}+w_{k+1} \Delta_{k+1}+\cdots+w_{t} \Delta_{t}$ for the normalized elements $\Lambda_{k}$ of the shortest path $\Gamma_{\min }(\Lambda, M)$.

We can even specify an actual (noneffective) formula for the permutations $w_{i}$ appearing in $T(\Lambda)^{\nearrow}=\Delta_{0}+w_{1} \Delta_{1}+w_{2} \Delta_{2}+\cdots+w_{t} \Delta_{t}$, attached to the shortest path

$$
\Lambda \stackrel{z^{\Delta_{1}}}{\longrightarrow} \Lambda_{1} \stackrel{z^{\Delta_{2}}}{\longrightarrow} \Lambda_{2} \longrightarrow \cdots \stackrel{z^{\Delta_{t-1}}}{\longrightarrow} \Lambda_{t-1} \stackrel{z^{\Delta_{t}}}{\longrightarrow} M_{\Lambda} \stackrel{z^{\Delta_{0}}}{\longrightarrow} M
$$

namely,

$$
w_{i}=\rho\left(\mathrm{HN}_{\Lambda_{1}}, \mathrm{HN}_{\Lambda}\right) \cdots \rho\left(\mathrm{HN}_{\Lambda_{i-1}}, \mathrm{HN}_{\Lambda_{i-2}}\right) \rho\left(\boldsymbol{F}^{\Lambda_{i}}(\Lambda), \boldsymbol{H}_{\Lambda_{i}}^{\Lambda_{i-1}}\right) .
$$

The formula cannot be reduced as in Corollary 29 since there is not necessarily an apartment adapted for three such consecutive flags.

3.2.2. The abacus. Theorem 33 has a nice combinatorial interpretation in terms of Young diagrams. For any integer sequence $D=\left(d_{1}, \ldots, d_{n}\right) \in \mathbb{Z}^{n}$, let $D=$ $\Delta_{0}+\cdots+\Delta_{t}$ be the elementary splitting of $D$. For $w=\left(w_{1}, w_{2}, \ldots, w_{t}\right) \in\left(S_{n}\right)^{t}$, let $w(D)=\Delta_{0}+w_{1} \Delta_{1}+w_{2} \Delta_{2}+\cdots+w_{t} \Delta_{t}$. The abacus of $D$ is the set of sequences

$$
\operatorname{ab}(D)=\left\{A \in \mathbb{Z}^{n} \mid \exists w \in\left(S_{n}\right)^{t}, A=w(D)\right\} .
$$

The name comes from the following analogy. Let $Y(D)$ be the Young diagram with $n$ rows whose respective lengths are the elements of $D_{0}=D-\min D \in \mathbb{N}^{n}$, assumed to be arranged in increasing order (rows of length 0 are included). Assume from now on that $\min D=0$. Let $d_{i}^{*}=\left|\left\{1 \leqslant j \leqslant n \mid d_{j} \geqslant i\right\}\right|$ be the number of boxes in the $i$-th column of $Y(D)$. The sequence $D^{*}=\left(d_{1}^{*}, \ldots, d_{t}^{*}\right)$ where $t=\max D$ is the dual sequence of $D$. The elementary splitting of $D$ is given by $\Delta_{i}=\left(0_{n-d_{i}^{*}}, I_{d_{i}^{*}}\right)$. In view of Lemma 19 , put

$$
\ell(D)=\sum_{i=1}^{t-1}\left(n-d_{i}^{*}\right) d_{i+1}^{*} .
$$

Any sequence $A$ in the abacus $\operatorname{ab}(D)$ of $D \in \mathbb{Z}^{n}$ comes from a box diagram obtained from $Y(D)$ by allowing to move some boxes only vertically inside the 

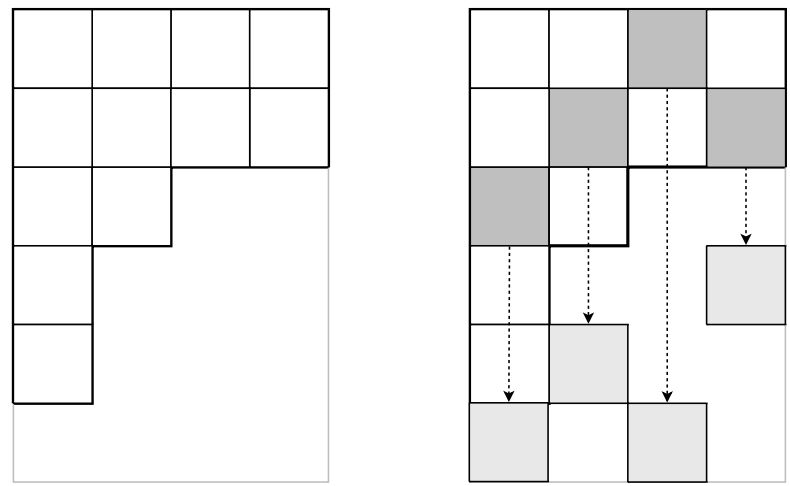

Figure 1. The Young diagram $Y(D)$ of the sequence $D=$ $(0,1,1,2,4,4)$, and an element $A=(2,2,2,1,2,3) \in \mathrm{ab}(D)$, featuring the moved boxes (in shades of gray), so that $A^{\nearrow}=$ $(1,2,2,2,2,3)$. The complement (thin gray line) corresponds to the sequence $D^{0}=(0,0,2,3,3,4)$, and the bijection from Lemma 35 , to reversing the arrows.

whole corresponding column of length $n$. The diagram thus obtained, that we call an abacus diagram, can have nonadjacent boxes (as shown in Figure 1). For any such diagram, the sequence $\left(a_{1}, \ldots, a_{n}\right)$ of number of boxes contained in each of the $n$ rows is the required element of $\mathrm{ab}(D)$.

Lemma 34. Let $D=\Delta_{1}+\cdots+\Delta_{t}$ be the elementary decomposition of $D \in\left(\mathbb{Z}^{n}\right)^{\nearrow}$, and let $A=w_{1} \Delta_{1}+\cdots+w_{t} \Delta_{t} \in \mathrm{ab}(D)$. There exist $w_{1}^{\prime}, \ldots, w_{t}^{\prime} \in S_{n}$ such that

$$
w_{1}^{\prime} \Delta_{1}+\cdots+w_{i}^{\prime} \Delta_{i}=\left(w_{1} \Delta_{1}+\cdots+w_{i} \Delta_{i}\right)^{\nearrow} \text { for all } 1 \leqslant i \leqslant d .
$$

Proof. We proceed by induction on the number $s$ of columns in $Y(D)$. Let $Y$ be the Young diagram for $D=\left(d_{1}, \ldots, d_{n}\right)$ and $\tilde{Y}$ the one obtained from $Y$ by erasing the last column, i.e., corresponding to the sequence $\widetilde{T}=\left(\Delta_{1}, \ldots, \Delta_{t-1}\right)$. Let $\widetilde{D}=\left(\tilde{d}_{1}, \ldots, \tilde{d}_{n}\right)$ be the associated sequence. Then we have $d_{i}=\tilde{d}_{i}$ for $1 \leqslant i \leqslant n-d_{t}^{*}$ and $d_{i}=\tilde{d}_{i}+1$ for $n-d_{t}^{*}+1 \leqslant i \leqslant n$. An element $A \in \operatorname{ab}(D)$ given by the permutations $w=\left(w_{2}, \ldots, w_{t}\right)$ corresponds uniquely to the pair $\left(\widetilde{A}, w_{t}\right)$ where $\widetilde{A} \in \operatorname{ab}(\widetilde{D})$ is given by the restriction $\widetilde{w}=\left(w_{2}, \ldots, w_{t-1}\right)$.

The claim is clear for $t=1$, so assume that it is established for any $\widetilde{D}$ such that $\Delta \widetilde{D} \leqslant t-1$. We have $D=\Delta_{1}+\cdots+\Delta_{t}=\widetilde{D}+\Delta_{t}$ where $\widetilde{D}=\Delta_{1}+\cdots+\Delta_{t-1}$ is the elementary decomposition of $\widetilde{D}$. Let $A \in \underset{\mathrm{ab}}{\mathrm{a}}(D)$ be described by the number $a_{i}$ of boxes in the $i$-th row for $1 \leqslant i \leqslant n$, and let $\tilde{A}=\left(\tilde{a}_{1}, \ldots, \tilde{a}_{n}\right)$ be the restriction of $A$ to the $t-1$ first columns. Let $\mathscr{F}=\left\{i \mid a_{i}=\tilde{a}_{i}+1\right\}$. Note that $\mathscr{F}=w_{t}\left(\left\{n-d_{t}^{*}+1, \ldots, n\right\}\right)$, and thus $|\mathscr{F}|=d_{t}^{*}$. According to the assumption, we can find $w_{1}^{\prime}, \ldots, w_{t-1}^{\prime}$ such that $w_{1}^{\prime} \Delta_{1}+\cdots+w_{i}^{\prime} \Delta_{i}=\left(w_{1} \Delta_{1}+\cdots+w_{i} \Delta_{i}\right)^{\nearrow}$ for all $1 \leqslant i \leqslant t-1$. In particular, 
we can assume that $\tilde{a}_{1} \leqslant \cdots \leqslant \tilde{a}_{n}$. If there exists an index $i$ such that $a_{i}>a_{i+1}$ holds, then we have necessarily $\tilde{a}_{i}=\tilde{a}_{i+1}=a_{i+1}$ and $a_{i}=\tilde{a}_{i}+1$, so $i \in \mathscr{g}$ and $i+1 \notin \mathscr{g}$. Exchanging $i$ and $i+1$ will not change the resulting sequence $A$ when reordered, so one can avoid the inversion by putting $w_{t}^{\prime}=(i, i+1) w_{t}$, so that $i \notin \mathscr{J}$ and $i+1 \in \mathscr{g}$ instead. By repeating this procedure for all the inverted indices, we get the claimed result for $t$.

For an $n \times t$ rectangular matrix $M=\left(M_{i, j}\right)$, define the row sum vector

$$
r(M)=\left(r_{1}, \ldots, r_{t}\right), \quad r_{j}=\sum_{i=1}^{n} M_{i, j},
$$

and column sum vector

$$
c(M)=\left(c_{1}, \ldots, c_{n}\right), \quad c_{i}=\sum_{j=1}^{t} M_{i, j} .
$$

An element $A \in \mathrm{ab}(D)$ in the abacus of $D$ can also be seen as a $(0,1)$ rectangular $n \times t$ matrix $\mathscr{A}=\left(A_{i, j}\right)$ where $A_{i, j}=1$ whenever $i \in w_{j}\left(\left\{n-d_{j}^{*}+1, \ldots, n\right\}\right)$ holds. This matrix $\mathscr{A}$ has row sum vector $r(\mathscr{A})=D^{*}$, and column sum vector $c(\mathscr{A})=A$. Recall that for two sequences $p=\left(p_{1}, \ldots, p_{n}\right)$ and $q=\left(q_{1}, \ldots, q_{t}\right)$ having the same sum, one says that $p$ dominates $q$ when $\sum_{i=1}^{k} q_{i} \leqslant \sum_{i=1}^{k} p_{i}$ for all integers $k$, where one completes the missing elements with 0 . The following lemma sums up the behavior of the quantities introduced in Section 2.1 under the abacus transformations.

Lemma 35. Let $D \in \mathbb{Z}^{n}$.

(i) The maps max, $\Delta, i, \ell$ and $\|\cdot\|$ all attain their maximum over $\operatorname{ab}(D)$ at $D$. The map min attains its minimum at $D$.

(ii) For any sequence $A \in \mathrm{ab}(D)$, the following hold:

(a) $\max A=\max D \Longleftrightarrow i(A)=i(D)$,

(b) $\Delta A=\Delta D \Longleftrightarrow \max A=\max D$ and $\min A=\min D$,

(c) $\|A\|=\|D\| \Longleftrightarrow \ell(A)=\ell(D) \Longleftrightarrow A^{\nearrow}=D^{\nearrow}$.

Moreover, the map $A \mapsto \max D-A$ is a bijection between $\operatorname{ab}\left(D_{0}\right)$ and $\operatorname{ab}\left(D^{0}\right)$.

Proof. We will only prove the claims about $\ell(D)$ and $\|D\|$, since the others are easily derived from their definitions. Although the assertions are similar, the methods of proof will be different. Let us start with $\ell$. Assume without loss of generality that $\min D=0$. According to Lemma 34, one can also assume that $A=A^{\nearrow}$ holds. Define the integers $k_{i}$ by induction as $k_{1}=a_{1}^{*}-d_{1}^{*}$ and $k_{i}=a_{i}^{*}-d_{i}^{*}+k_{i-1}$ for $i \geqslant 2$. Since $A$ can be seen as a $(0,1)$ matrix $\mathscr{A}$ with $r(\mathscr{A})=D^{*}$ and $c(\mathscr{A})=A$, the GaleRyser theorem [Krause 1996] implies that $c(\mathscr{A})^{*}=A^{*}$ dominates $r(\mathscr{A})=D^{*}$. 
Therefore

$$
\sum_{i=1}^{k} d_{i}^{*} \leqslant \sum_{i=1}^{k} a_{i}^{*}
$$

for all integers $k$. Hence $k_{i} \geqslant 0$ for all $i$. Putting $k_{0}=k_{t}=0$, we then have $a_{i}^{*}=d_{i}^{*}-k_{i-1}+k_{i}$ for $1 \leqslant i \leqslant d$. According to the definition, we have

$$
\begin{aligned}
\ell(A) & =\sum_{i=1}^{t-1}\left(n-a_{i}^{*}\right) a_{i+1}^{*}=\sum_{i=1}^{t-1}\left(n-d_{i}^{*}+k_{i-1}-k_{i}\right)\left(d_{i+1}^{*}-k_{i}+k_{i+1}\right) \\
& =\sum_{i=1}^{t-1}\left(n-d_{i}^{*}\right) d_{i+1}^{*}+\left(k_{i-1}-k_{i}\right)\left(d_{i+1}^{*}-k_{i}+k_{i+1}\right)-\left(k_{i}-k_{i+1}\right)\left(n-d_{i}^{*}\right) \\
& =\ell(D)-\sum_{i=1}^{t-1}\left(k_{i}-k_{i+1}\right)\left(n-d_{i}^{*}+d_{i+1}^{*}+k_{i+1}-k_{i}\right)
\end{aligned}
$$

After some algebraic manipulation, we get

$$
\begin{aligned}
\ell(A)-\ell(D)= & -k_{1}\left(n-d_{1}^{*}+d_{2}^{*}-k_{1}+k_{2}-\left(d_{3}^{*}-k_{2}+k_{3}\right)\right) \\
& \cdots-\sum_{i=2}^{t-2} k_{i}\left(d_{i+1}^{*}-k_{i}+k_{i+1}-\left(d_{i+2}^{*}-k_{i+1}+k_{i+2}\right)+d_{i-1}^{*}-d_{i}^{*}\right) \\
& \cdots-k_{t-1}\left(d_{t}^{*}-k_{t-1}+k_{t}+d_{t-2}^{*}-d_{t-1}^{*}\right) \\
= & -k_{1}\left(n-d_{1}^{*}+a_{2}^{*}-a_{3}^{*}\right) \cdots-\sum_{i=2}^{t-2} k_{i}\left(a_{i+1}^{*}-a_{i+2}^{*}+d_{i-1}^{*}-d_{i}^{*}\right) \\
& \cdots-k_{t-1}\left(a_{t}^{*}+d_{t-2}^{*}-d_{t-1}^{*}\right) .
\end{aligned}
$$

Since both sequences $\left(d_{i}^{*}\right)$ and $\left(a_{i}^{*}\right)$ are nonincreasing, we get $\ell(A) \leqslant \ell(D)$. Moreover, if $\ell(A)=\ell(D)$ holds, then all these terms must be zero. Let us prove then by induction that $k_{i}=0$ for all $i$. If $k_{1} \neq 0$, then one has $n=d_{1}^{*}$ and $a_{2}^{*}=a_{3}^{*}$. Since $a_{1}^{*} \geqslant d_{1}^{*}$, one must therefore have $a_{1}^{*}=n$, so $k_{1}=0$ holds, and hence we get $d_{1}^{*}=a_{1}^{*}$. Assume now that $k_{j}=0$ and $a_{j}^{*}=d_{j}^{*}$ hold for $j \leqslant i-1$. If $k_{i} \neq 0$ then one has $a_{i}^{*}>d_{i}^{*}$ and $d_{i-1}^{*}=d_{i}^{*}$ (and $a_{i+1}^{*}=a_{i+2}^{*}$ also). But then $d_{i-1}^{*}=d_{i}^{*}=a_{i-1}^{*} \geqslant a_{i}^{*} \geqslant d_{i}^{*}$, so we have $d_{i-1}^{*}=d_{i}^{*}=a_{i-1}^{*}=a_{i}^{*}$. Thus $k_{i}=a_{i}^{*}-d_{i}^{*}+k_{i-1}=0$, and so the result for $\ell$ is established.

Let us turn now to $\|\cdot\|$. We can assume here that $w_{1}=\mathrm{id}$. We proceed by induction on the number $t$ of columns in the Young diagram $Y(D)$. Like in the proof of the previous result, the restricted column sequence $T^{\prime}=\left(\Delta_{1}, \ldots, \Delta_{t-1}\right)$ corresponds to the Young diagram $Y\left(D^{\prime}\right)$ of $D^{\prime}=\left(d_{1}^{\prime}, \ldots, d_{n}^{\prime}\right)$ with $d_{i}=d_{i}^{\prime}$ for $1 \leqslant i \leqslant n-d_{t}^{*}$ and $d_{i}=d_{i}^{\prime}+1$ for $n-d_{t}^{*}+1 \leqslant i \leqslant n$. By the König-Huygens 
identity, we have

$$
\begin{aligned}
\|D\| & =\sum_{i=1}^{n}\left(d_{i}-\frac{\operatorname{Tr} D}{n}\right)^{2}=\sum_{i=1}^{n} d_{i}^{2}-\frac{(\operatorname{Tr} D)^{2}}{n} \\
& =\sum_{i=1}^{n-d_{t}^{*}}\left(d_{i}^{\prime}\right)^{2}+\sum_{i=n-d_{t}^{*}+1}^{n}\left(d_{i}^{\prime}+1\right)^{2}-\frac{\left(\operatorname{Tr} D^{\prime}+d_{t}^{*}\right)^{2}}{n} \\
& =\sum_{i=1}^{n}\left(d_{i}^{\prime}\right)^{2}-\frac{\left(\operatorname{Tr} D^{\prime}\right)^{2}}{n}+d_{t}^{*}+2 \sum_{i=n-d_{t}^{*}+1}^{n} d_{i}^{\prime}-\frac{2 d_{t}^{*} \operatorname{Tr} D^{\prime}+\left(d_{t}^{*}\right)^{2}}{n} \\
& =\left\|D^{\prime}\right\|+d_{t}^{*}+2 d_{t}^{*}(t-1)-\frac{2 d_{t}^{*} \operatorname{Tr} D^{\prime}+\left(d_{t}^{*}\right)^{2}}{n} .
\end{aligned}
$$

Let $A=w(D) \in \operatorname{ab}(D)$ with $w=\left(w_{2}, \ldots, w_{t}\right)$ and $A^{\prime}=w^{\prime}\left(D^{\prime}\right) \in \operatorname{ab}\left(D^{\prime}\right)$ where $w^{\prime}=\left(w_{2}, \ldots, w_{t-1}\right)$.

For $t=1$, the claim is clear, for $\|w(D)\|=\|D\|$ and $w(D)^{\nearrow}=D$ for any $w \in\left(S_{t}\right)^{n}$. Assume then that for any diagram $Y^{\prime}=Y\left(D^{\prime}\right)$ with at most $t-1$ columns, we have $\left\|A^{\prime}\right\| \leqslant\left\|D^{\prime}\right\|$ for $A^{\prime} \in \mathrm{ab}\left(D^{\prime}\right)$, and $\left\|A^{\prime}\right\|=\left\|D^{\prime}\right\|$ if and only if $\left(A^{\prime}\right)^{\nearrow}=D^{\prime}$. Let $Y=Y(D)$ have $t$ columns. Let $A \in \mathrm{ab}(D)$ be described by the number $a_{i}$ of boxes in the $i$-th row for $1 \leqslant i \leqslant n$, and let $A^{\prime}=\left(a_{1}^{\prime}, \ldots, a_{n}^{\prime}\right)$ be the restriction of $A$ to the $t-1$ first columns. Let again $\mathscr{E}=\left\{i \mid a_{i}=a_{i}^{\prime}+1\right\}$. Then one has

$$
\begin{aligned}
\|A\|= & \sum_{i=1}^{n} a_{i}^{2}-\frac{(\operatorname{Tr} A)^{2}}{n}=\sum_{i \in \mathscr{F}}\left(a_{i}^{\prime}+1\right)^{2}+\sum_{i \notin \mathscr{F}}^{n}\left(a_{i}^{\prime}\right)^{2}-\frac{\left(\operatorname{Tr} A^{\prime}+d_{t}^{*}\right)^{2}}{n} \\
& =\sum_{i=1}^{n}\left(a_{i}^{\prime}\right)^{2}-\frac{\left(\operatorname{Tr} A^{\prime}\right)^{2}}{n}+|\mathscr{}|+2 \sum_{i \in \mathscr{F}}^{n} a_{i}^{\prime}-\frac{2 d_{t}^{*} \operatorname{Tr} A^{\prime}+\left(d_{t}^{*}\right)^{2}}{n} \\
& =\left\|A^{\prime}\right\|+d_{t}^{*}-\frac{2 d_{t}^{*} \operatorname{Tr} A^{\prime}+\left(d_{t}^{*}\right)^{2}}{n}+2 \sum_{i \in \mathscr{F}}^{n} a_{i}^{\prime} .
\end{aligned}
$$

By construction, we have $\operatorname{Tr} A^{\prime}=\operatorname{Tr} D^{\prime}$, so, under the induction assumption, we get

$\|A\| \leqslant\left\|D^{\prime}\right\|+d_{t}^{*}-\frac{2 d_{t}^{*} \operatorname{Tr} D^{\prime}+\left(d_{t}^{*}\right)^{2}}{n}+2 \sum_{i \in \mathscr{F}}^{n} a_{i}^{\prime}=\|D\|+2\left(\sum_{i \in \mathscr{F}} a_{i}^{\prime}-d_{t}^{*}(t-1)\right)$.

Since $a_{i}^{\prime} \leqslant t-1=\max D^{\prime}$ holds by construction, and $|\mathscr{F}|=d_{t}^{*}$, we get $\|A\| \leqslant\|D\|$. Moreover, $\|A\|=\|D\|$ can only happen when $a_{i}^{\prime}=t-1$ for $i \in \mathscr{F}$. In this case, 
we get

$$
\left\|A^{\prime}\right\|=\|D\|-\left(d_{t}^{*}+2 d_{t}^{*}(t-1)-\frac{2 d_{t}^{*} \operatorname{Tr} D^{\prime}+\left(d_{t}^{*}\right)^{2}}{n}\right)=\left\|D^{\prime}\right\| .
$$

By the induction assumption, we have $\left(A^{\prime}\right)^{\nearrow}=D^{\prime}$, and by the definition of $\mathscr{F}$, we get $a_{i}=a_{i}^{\prime}+1=d=\max D$ for $i \in \mathscr{F}$. Therefore, we get $d_{t}^{*}$ elements in $A$ which are equal to $d$, and hence $A^{\nearrow}=D$.

3.2.3. Local criteria for $B G$ trivializations. In this section, we use the fact that the type $T(\Lambda)$ is an element of $\operatorname{ab}\left(\mathbf{E D}_{\Lambda}(M)\right)$ for any trivial $M \in \Lambda^{0}$ to derive local criteria satisfied by the BG trivializations. Let $d\left(\Lambda, \Lambda^{0}\right)=\min _{M \in \Lambda^{0}} d(\Lambda, M)$.

Lemma 36. Let $\Lambda \in \Lambda$ be a lattice. For any $M \in \Lambda^{0}$, we have $d(\Lambda, M)=d\left(\Lambda, \Lambda^{0}\right) \Longleftrightarrow v_{\Lambda}(M)=\max _{\tilde{M} \in \Lambda^{0}} v_{\Lambda}(\tilde{M})$ and $v_{M}(\Lambda)=\max _{\tilde{M} \in \Lambda^{0}} v_{\tilde{M}}(\Lambda)$. If $M \in \operatorname{BG}(\Lambda)$, then $\delta(\Lambda, M)=\min _{\tilde{M} \in \mathbf{\Lambda}^{0}} \delta(\Lambda, \tilde{M})$ for $\delta \in\{d, \boldsymbol{d}, \ell\}$.

Proof. Let $\Lambda^{0} \ni \tilde{M} \stackrel{z^{D}}{\longrightarrow} \Lambda$ represent the elementary divisors of an arbitrary trivialization $\tilde{M}$ of $\Lambda$. The BG algorithm of Section 3.2.1 can be applied to $\tilde{M}$ to obtain $M \in \mathrm{BG}(\Lambda)$, as in the following scheme, where $A=w(D) \in \mathrm{ab}(D)$ :

$$
\Lambda^{0} \ni \tilde{M} \stackrel{z^{D}}{\longleftarrow} \stackrel{z^{A}}{\longrightarrow} M \in \operatorname{BG}(\Lambda) .
$$

By definition, we have $T(\Lambda) \cong A$. By Lemma 35 , for any $\tilde{M} \in \Lambda^{0}$, we have $v_{\Lambda}(\tilde{M})=\min D \leqslant \min A=v_{\Lambda}(M)$ and $v_{\tilde{M}}(\Lambda)=-\max D \leqslant-\max A=v_{M}(\Lambda)$. This holds for any trivial $\tilde{M}$; hence, for any $\mathrm{BG}$ trivialization $M \in \operatorname{BG}(\Lambda)$, we get

$$
v_{\Lambda}(M)=\max _{\tilde{M} \in \Lambda^{0}} v_{\Lambda}(\tilde{M}) \quad \text { and } \quad v_{M}(\Lambda)=\max _{\tilde{M} \in \Lambda^{0}}\left(v_{\tilde{M}}(\Lambda)\right) .
$$

On the other hand, we have, for any $\tilde{M} \in \Lambda^{0}$,

$d(\Lambda, \tilde{M})=-v_{\Lambda}(\tilde{M})-v_{\tilde{M}}(\Lambda) \geqslant-v_{\Lambda}(M)-v_{M}(\Lambda)=d(\Lambda, M)$ for $M \in \operatorname{BG}(\Lambda)$.

The rest in a direct consequence of Lemma 35 .

Proposition 37. Let $\Lambda \in \Lambda$. Then

$$
T(\Lambda)=\max _{\preceq_{\text {lex }}}\left\{\mathbf{E D}_{\Lambda}(M)^{\nearrow} \mid M \in \Lambda^{0}\right\}=\min _{\preceq_{\text {lexrev }}}\left\{\mathbf{E D}_{\Lambda}(M)^{\nearrow} \mid M \in \Lambda^{0}\right\} .
$$

Proof. Let $M \in \Lambda^{0}$, and let $D=\left(d_{1}, \ldots, d_{n}\right)=\mathbf{E D}_{\Lambda}(M)^{\nearrow}$. By Theorem 33, there exists $w=\left(w_{1}, \ldots, w_{t}\right) \in\left(S_{n}\right)^{t}$ such that $w(D) \cong T(\Lambda)$, and we can, by Lemma 34, ensure that $w(D)=T(\Lambda)^{\nearrow}$. Representing $D$ as its Young diagram $Y(D)$, the sequence $T(\Lambda)$ is given by an abacus diagram $A \in \operatorname{ab}(D)$ derived from $Y(D)$ by moving boxes vertically (downwards), and such that the number 
of boxes $t_{i}$ in row $i$ weakly increases with the row index (i.e., $t_{i+1} \geqslant t_{i}$ ). Then we have $d_{1}=v_{\Lambda}(M) \leqslant t_{1}=\max _{\tilde{M} \in \Lambda^{0}} v_{\Lambda}(\tilde{M})$. Suppose that $d_{1}=t_{1}$, and assume that $d_{2}>t_{2}$ holds. Accordingly some boxes from the second row must be lowered. Therefore, we necessarily have $t_{1}>d_{1}$. This contradicts the maximality assumption on $v_{\Lambda}(M)$. Thus $d_{2} \leqslant t_{2}$, and so $t_{2}=\max _{M \in \mathbf{\Lambda}^{0}, v_{\Lambda}}(M)=t_{1} d_{2}^{\Lambda}(M)$. Let $i=\max \left\{j \mid d_{k}=t_{k}\right.$ for $\left.k \leqslant j\right\}$ and assume that $d_{i+1}>t_{i+1}$ holds. The same argument holds and shows that $d_{i+1} \leqslant t_{i+1}$. Therefore we have proved that $t_{i}=\max _{M \in \mathbf{\Lambda}^{0}, d_{k}^{\Lambda}(M)=t_{k}, 1 \leqslant k \leqslant i-1} d_{i}^{\Lambda}(M)$. A similar argument starting from $d_{n}=-\max v_{M}(\Lambda)$ proves the second relation.

This result establishes the first part of Theorem 25. The proof will be complete when we prove that $M$ is indeed a BG trivialization of $\Lambda$ if and only if $M \in \Lambda^{0}$ and $\mathbf{E D}_{\Lambda}(M) \cong T(\Lambda)$. This will be established in the next section.

3.3. The permutation lemma. In the previous section, we showed how to construct a lattice $M$ whose global form $Y_{M}$ contains a Smith basis for a given lattice $\Lambda$. The following result allows us to give a fairly complete geometric view of the set of $\mathrm{BG}$ trivializations. We recall that a principal minor of a matrix $A \in \mathfrak{g l}_{n}(\mathbb{C})$ is a minor $A_{I, I}$ where $I \subset[n]$ obtained by deleting rows and columns whose indices are not elements of $I$.

Proposition 38 (permutation lemma). Let $D=\left(d_{1}, \ldots, d_{n}\right) \in \mathbb{Z}^{n}$ be an integer sequence and $P \in \mathrm{GL}_{n}(\mathbb{C} \llbracket t \rrbracket)$ a lattice gauge.

(1) (Bolibrukh) There exist a permutation $\tau \in S_{n}$ and a lattice gauge $\tilde{P} \in$ $\mathrm{GL}_{n}(\mathbb{C} \llbracket t \rrbracket)$ such that

$$
\Pi=t^{-D} P^{-1} t^{\tau D} \tilde{P} \in \mathrm{GL}_{n}\left(\mathbb{C}\left[t^{-1}\right]\right),
$$

where $t^{D}=\operatorname{diag}\left(t^{d_{1}}, \ldots, t^{d_{n}}\right)$ and $\tau D=\left(d_{\tau(1)}, \ldots, d_{\tau(n)}\right)$.

(2) There exists moreover a lattice gauge $Q \in \mathrm{GL}_{n}(\mathcal{O})$ such that $t^{D} \Pi=Q t^{D}$.

(3) Furthermore, one can choose $\sigma=1$ in item (1) if and only if all principal minors of $P(0)$ indexed by the elements of the ascending flag $\boldsymbol{D}^{\nearrow}$ are nonzero.

We will give a self-contained proof of this result, following for the first item, due to Bolibrukh, the same lines as the proof of this lemma given in [Ilyashenko and Yakovenko 2007]. Item (2) is, up to our knowledge, new, as well as the necessity statement in (3) (sufficiency appears in the cited work). The proof proceeds by induction, using the following simple lemma.

Lemma 39. Let $d \leqslant n$ and

$$
T=\left(\begin{array}{cc}
I_{d} & 0 \\
0 & 0_{n-d}
\end{array}\right)
$$


Let $H=\left(\begin{array}{ll}A & B \\ C & D\end{array}\right) \in \mathrm{GL}_{n}(\mathbb{C} \llbracket t \rrbracket)$ be a lattice gauge matrix, decomposed as a $2 \times 2$-block matrix according to the blocks of $T$. If $\operatorname{det} A(0) \neq 0$, then there exists a monopole gauge matrix

$$
\Pi=\left(\begin{array}{cc}
I_{d} & t^{-1} \widetilde{\Pi} \\
0 & I_{n-d}
\end{array}\right)
$$

with $\widetilde{\Pi}$ a constant matrix, such that $\widetilde{H}=t^{-T} H t^{T} \Pi$ is a lattice gauge matrix; that is, $\tilde{H} \in \mathrm{GL}_{n}(\mathrm{O})$.

Proof. For simplicity, put $M_{0}=M(0)$ for a holomorphic matrix $M$. One checks that putting $\widetilde{\Pi}=-A_{0}^{-1} B_{0}$, we have

$$
\widetilde{H}=t^{-T} H t^{T} \Pi=\left(\begin{array}{cc}
A & \widetilde{B} \\
t C & \widetilde{D}
\end{array}\right),
$$

where

$$
\widetilde{B}=t^{-1}(B+A \widetilde{\Pi}) \quad \text { and } \quad \widetilde{D}=D+C \widetilde{\Pi} .
$$

By construction, the residue of $\widetilde{B}$ is equal to $B_{0}-A_{0} A_{0}^{-1} B_{0}=0$, and hence $\widetilde{B}$ is holomorphic; therefore $\widetilde{H}$ also is. To check that $\widetilde{H} \in \mathrm{GL}_{n}(\mathcal{O})$, it is sufficient to check the invertibility of

$$
\widetilde{H}_{0}=\left(\begin{array}{cc}
A_{0} & \widetilde{B}_{0} \\
0 & D_{0}-C_{0} A_{0}^{-1} B_{0}
\end{array}\right) .
$$

By assumption $A_{0}$ is invertible, and it is well-known that the Schur complement $D-C A^{-1} B$ is invertible when $\left(\begin{array}{cc}A & B \\ C & D\end{array}\right) \in \mathrm{GL}_{n}(\mathbb{C})$ and $A$ both are so.

Note that the upper-left block of $H$ appears unchanged in $\widetilde{H}$. Note also that

$$
\bar{H}=t^{T} \Pi=\left(\begin{array}{cc}
t I_{d} & \widetilde{\Pi} \\
0 & I_{n-d}
\end{array}\right) .
$$

Geometrically, we can summarize the construction of Lemma 39 as the following scheme.

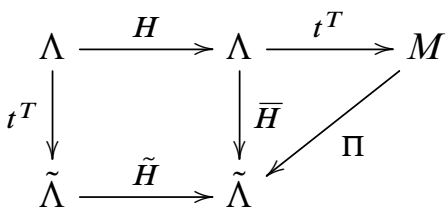

We need two technical lemmata before giving the actual proof of the permutation lemma. Let $D$ denote the integer sequence

$$
(\underbrace{d_{1}, \ldots, d_{1}}_{n_{1} \text { times }}, \ldots, \underbrace{d_{s}, \ldots, d_{s}}_{n_{s} \text { times }})
$$


with $d_{i}>d_{i+1}$. We say that a matrix $H$ is strongly $D$-parabolic if it has the form

$$
H=\left(\begin{array}{ccc}
t^{d_{1}} I_{n_{1}} & \cdots & P_{i j} \\
& t^{d_{2}} I_{n_{2}} & \vdots \\
0 & & t^{d_{s}} I_{n_{s}}
\end{array}\right),
$$

where $P_{i j}$ is a $n_{i} \times n_{j}$ polynomial matrix in $t$ satisfying $\operatorname{deg} P_{i j}<d_{i}$ and $v\left(P_{i j}\right) \geqslant d_{j}$.

Lemma 40. Let $H$ be strongly $D$-parabolic, and let

$$
H^{\prime}=\left(\begin{array}{cc}
t I_{m} & \widetilde{\Pi} \\
0 & I_{n-m}
\end{array}\right),
$$

where $\widetilde{\Pi}$ is a constant matrix and $m \leqslant n_{1}$. Then the product $H H^{\prime}$ is strongly $D^{\prime}$-parabolic, where $D^{\prime}=(\underbrace{d_{1}+1, \ldots, d_{1}+1}_{m \text { times }}, \underbrace{d_{1}, \ldots, d_{1}}_{n_{1}-m \text { times }}, \ldots, \underbrace{d_{s}, \ldots, d_{s}}_{n_{s} \text { times }})$.

Proof. Let $\bar{D}=(\underbrace{d_{2}, \ldots, d_{2}}_{n_{2} \text { times }}, \ldots, \underbrace{d_{s}, \ldots, d_{s}}_{n_{s} \text { times }})$. The matrix $H$ can be written as

$$
H=\left(\begin{array}{cc}
t^{d_{1}} I_{n_{1}} & P \\
0 & \bar{H}
\end{array}\right)
$$

where $\bar{H}$ is strongly $\bar{D}$-parabolic, and $P=\left(P_{2} \cdots P_{S}\right)$ where the blocks $P_{i}$ satisfy $\operatorname{deg} P_{i}<d_{1}$ and $v\left(P_{i}\right) \geqslant d_{i}$. Then, if $m=n_{1}$, the product $H H^{\prime}$ is simply

$$
H H^{\prime}=\left(\begin{array}{cc}
t^{d_{1}+1} I_{n_{1}} & t^{d_{1}} \tilde{\Pi}+P \\
0 & \bar{H}
\end{array}\right) .
$$

Otherwise, we split the matrices in $3 \times 3$-blocks, as

$$
\begin{aligned}
H H^{\prime} & =\left(\begin{array}{ccc}
t^{m} I_{m} & 0 & P_{1} \\
0 & t^{d_{1}} I_{n_{1}-m} & P_{2} \\
0 & 0 & \bar{H}
\end{array}\right)\left(\begin{array}{ccc}
t I_{m} & \widetilde{\Pi}_{1} & \widetilde{\Pi}_{2} \\
0 & I_{n_{1}-m} & 0 \\
0 & 0 & I_{n-n_{1}}
\end{array}\right) \\
& =\left(\begin{array}{ccc}
t^{d_{1}+1} I_{m} & t^{d_{1}} \widetilde{\Pi}_{1} & t^{d_{1}} \widetilde{\Pi}_{1}+P_{1} \\
0 & t^{d_{1}} I_{n_{1}-m} & P_{2} \\
0 & 0 & \bar{H}
\end{array}\right) .
\end{aligned}
$$

In both cases, we see that the product $H H^{\prime}$ is strongly $D^{\prime}$-parabolic as requested.

In the following lemma, we prove that the factorization in Proposition 38 exists if and only if the condition on the minors of the constant term holds.

Lemma 41. Let $P, Q \in \mathrm{GL}_{n}(\mathrm{O})$, and let

$$
D=(\underbrace{d_{1}, \ldots, d_{1}}_{n_{1} \text { times }}, \ldots, \underbrace{d_{s}, \ldots, d_{s}}_{n_{s} \text { times }})
$$


with $d_{i}>d_{i+1}$. Assume that $\Pi=z^{-D} P_{z}{ }^{D} Q \in \mathfrak{g l}_{n}(\mathbb{C} \llbracket 1 / z \rrbracket)$. Decomposing a matrix $M$ in blocks $M_{i, j}$ according to the multiplicities $\left(n_{1}, \ldots, n_{s}\right)$ of $D$, let

$$
P_{i}=\left(\begin{array}{ccc}
P_{i, i} & \cdots & P_{i, s} \\
\vdots & \ddots & \vdots \\
P_{s, i} & \cdots & P_{s, s}
\end{array}\right) \quad \text { and } \quad \widetilde{\Pi}_{i}=\left(\begin{array}{ccc}
\Pi_{i, 1}^{(0)} & \cdots & \Pi_{i, s}^{(0)} \\
\vdots & \ddots & \vdots \\
\Pi_{s, 1}^{(0)} & \cdots & \Pi_{s, s}^{(0)}
\end{array}\right)
$$

Then $\widetilde{\Pi}_{i}$ has maximal rank if and only if $P_{i}(0), \ldots, P_{S}(0)$ are all invertible.

Proof. We prove this result by induction. We put $M=\sum_{\ell \in \mathbb{Z}} M^{(\ell)} z^{\ell}$ for a formal series matrix, with $M^{(\ell)}=0$ when needed. Let $M_{i,(j: k)}=\left(\begin{array}{lll}M_{i, j} & \cdots & M_{i, k}\end{array}\right)$ for $j \leqslant k$, and $M_{i, \bullet}=M_{i,(1: s)}$, and put further

$$
\tilde{M}_{j}=\left(\begin{array}{c}
M_{j, \bullet}^{(0)} \\
M_{j+1, \bullet}^{(0)} \\
\vdots \\
M_{s, \bullet}^{(0)}
\end{array}\right) \quad \text { and } \quad M_{j}^{(\ell)}=\left(\begin{array}{c}
M_{j, \bullet}^{(\ell)} \\
M_{j+1, \bullet}^{\left(\ell+d_{j}-d_{j+1}\right)} \\
\vdots \\
M_{s, \bullet}^{\left(\ell+d_{j}-d_{s}\right)}
\end{array}\right) \text {, }
$$

so that

$$
M_{j}^{\prime(\ell)}=\left(\begin{array}{c}
M_{j, \bullet}^{(\ell)} \\
M_{j+1}^{\left(\ell+d_{j}-d_{j+1}\right)}
\end{array}\right) \text {. }
$$

Let $\check{P}=z^{-D} P z^{D}$. By construction, we have $\check{P}=\left(\check{P}_{i, j}\right)$ where

$$
\check{P}_{i, j}=P_{i, j} z^{d_{j}-d_{i}}=\sum_{\ell \geqslant 0} P_{i, j}^{(\ell)} z^{\ell+d_{j}-d_{i}}=\sum_{\ell \geqslant d_{j}-d_{i}} P_{i, j}^{\left(\ell+d_{i}-d_{j}\right)} z^{\ell} .
$$

Since $\Pi=\check{P} Q$ holds, we have $\Pi_{i, \bullet}=\sum_{i=1}^{s} \check{P}_{i, k} Q_{k, \bullet}$, with $Q_{k, \bullet}=\sum_{\ell \geqslant 0} Q_{k, \bullet}^{(\ell)} z^{\ell}$. After some algebra, we get

(12) $\Pi_{i, \bullet}=\sum_{t \leqslant 0}\left(\sum_{k=1}^{s} \sum_{\ell=d_{k}-d_{i}}^{t} P_{i, k}^{\left(\ell+d_{i}-d_{k}\right)} Q_{k, \bullet}^{(t-\ell)}\right) z^{t} \quad$ since $\Pi \in \mathfrak{g l}_{n}(\mathbb{C} \llbracket 1 / z \rrbracket)$.

We will establish the main claim (MC) of the lemma by proving simultaneously the following two additional results. If the assumption of the lemma holds, then we have

(A) $\widetilde{Q}_{j}$ is in the row span of $\widetilde{\Pi}_{j}$ for $i \leqslant j \leqslant s$,

(B) for $i \leqslant j \leqslant s$ and $\ell \leqslant d_{j-1}-d_{j}-1$, there exists a matrix $X_{j \ell}$ such that $Q_{j}^{(\ell)}=X_{j \ell} \widetilde{Q}_{j}$ 
Assume first that $i=s$. According to formula (12), we have

$$
\Pi_{s, \bullet}=\sum_{t \leqslant 0}\left(\sum_{k=1}^{s} \sum_{\ell=d_{k}-d_{s}}^{t} P_{s, k}^{\left(\ell+d_{s}-d_{k}\right)} Q_{k, \bullet}^{(t-\ell)}\right) z^{t} .
$$

Since $d_{k}-d_{s}>0$ for $k \neq s$, we have that $\Pi_{s, \bullet}$ is a constant, and the formula reduces to $t=0$ and $k=s$; thus $\Pi_{s, \bullet}^{(0)}=P_{s, s}^{(0)} Q_{s, \bullet}^{(0)}$. Since $\operatorname{rk} Q_{s, \bullet}^{(0)}=n_{s}$, we get

$$
\operatorname{rk} \Pi_{s, \bullet}^{(0)}=n_{s} \Longleftrightarrow \operatorname{det} P_{s, s}^{(0)} \neq 0,
$$

which establishes (MC) and (A) for $i=s$. For $0<t<d_{s-1}-d_{s}$, formula (12) reduces to

$$
\Pi_{s, \bullet}^{(t)}=\sum_{\ell=0}^{t} P_{s, s}^{(\ell)} Q_{s, \bullet}^{(t-\ell)}=0
$$

since $\Pi^{(t)}=0$ for $t>0$. We can arrange all these equations into the large matrix equation

$$
\left(\begin{array}{cccc}
P_{s, s}^{(0)} & 0 & \cdots & 0 \\
P_{s, s}^{(1)} & P_{s, s}^{(0)} & & \vdots \\
\vdots & & \ddots & \vdots \\
P_{s, s}^{\left(d_{s-1}-d_{s}-1\right)} & \cdots & \cdots & P_{s, s}^{(0)}
\end{array}\right)\left(\begin{array}{c}
Q_{s, \bullet}^{(0)} \\
Q_{s, \bullet}^{(1)} \\
\vdots \\
Q_{s, \bullet}^{\left(d_{s-1}-d_{s}-1\right)}
\end{array}\right)=\left(\begin{array}{c}
\Pi_{s, \bullet}^{(0)} \\
0 \\
\vdots \\
0
\end{array}\right) .
$$

It follows easily that $Q_{s, \bullet}^{(\ell)}$ is then a left multiple of $Q_{s, \bullet}^{(0)}$ for $0 \leqslant \ell \leqslant d_{s-1}-d_{s}-1$, and also for $\ell<0$, since $Q_{s, \bullet}^{(\ell)}=0$, which establishes the claim (B) for $i=s$.

Assume now that (MC), (A) and (B) hold for $i+1 \leqslant j \leqslant s$ and $\ell \leqslant d_{j-1}-d_{j}-1$. Formula (12) gives

$$
\begin{aligned}
\Pi_{j, \bullet}^{(t)} & =\sum_{k=1}^{s} \sum_{\ell=d_{k}-d_{j}}^{t} P_{j, k}^{\left(\ell+d_{j}-d_{k}\right)} Q_{k, \bullet}^{(t-\ell)} \\
& =\sum_{\ell \geqslant 0} \sum_{k=1}^{s} P_{j, k}^{(\ell)} Q_{k, \bullet}^{\left(t+d_{j}-d_{k}-\ell\right)},
\end{aligned}
$$

with the convention that $Q^{(\ell)}=0$ when $\ell<0$. Considering exponents $t=d_{i}-d_{j}+m$ for $i \leqslant j \leqslant s$ and $0 \leqslant m \leqslant d_{i-1}-d_{i}-1$, we get

$$
\begin{aligned}
\Pi_{j, \bullet}^{\left(d_{i}-d_{j}+m\right)} & =\sum_{\ell \geqslant 0} \sum_{k=i}^{s} P_{j, k}^{(\ell)} Q_{k, \bullet}^{\left(m+d_{i}-d_{k}-\ell\right)} \\
& =\sum_{\ell \geqslant 0} P_{j,(i: s)}^{(\ell)} Q_{i}^{(m-\ell)}
\end{aligned}
$$


since $k<i$ implies $m+d_{i}-d_{k}<0$. Therefore, we get

$$
\begin{aligned}
\Pi_{j, \bullet}^{\left(d_{i}-d_{j}+m\right)} \\
=\sum_{\ell \geqslant 0} P_{j, i}^{(\ell)} Q_{i, \bullet}^{(m-\ell)}+P_{j,(i+1: s)}^{(\ell)} Q_{i+1}^{\prime\left(m+d_{i}-d_{i+1}-\ell\right)} \\
=\sum_{\ell=0}^{m}\left(P_{j, i}^{(\ell)} Q_{i, \bullet}^{(m-\ell)}+P_{j,(i+1: s)}^{(\ell)} Q_{i+1}^{\left(m+d_{i}-d_{i+1}-\ell\right)}\right)+\sum_{\ell>m} P_{j,(i+1: s)}^{(\ell)} Q_{i+1}^{\prime\left(m+d_{i}-d_{i+1}-\ell\right)} \\
=\sum_{\ell=0}^{m} P_{j,(i: s)}^{(\ell)} Q_{i}^{(m-\ell)}+\sum_{\ell>m} P_{j,(i+1: s)}^{(\ell)} Q_{i+1}^{\left(m+d_{i}-d_{i+1}-\ell\right)} .
\end{aligned}
$$

According to the induction $Q_{i+1}^{\prime\left(m+d_{i}-d_{i+1}-\ell\right)}$ is a left multiple of $\widetilde{Q}_{i+1}$ for $\ell>m$, so we have

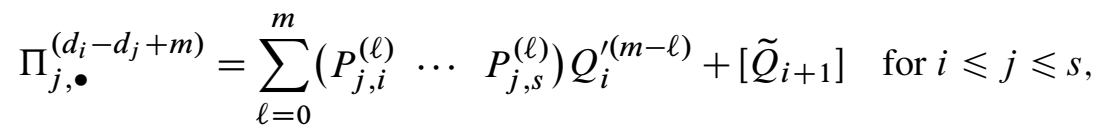

where we let, for notational simplicity, $A=B+[Q]$ mean "there exists a matrix $X$ such that $A-B=X Q$ ". Assume $m=0$ first. The equation for $j=i$ is then

$$
\Pi_{i, \bullet}^{(0)}=P_{i, i}^{(0)} Q_{i, \bullet}^{(0)}+\left(P_{i, i+1}^{(0)} \cdots P_{i, s}^{(0)}\right) Q_{i+1}^{\left(d_{i}-d_{i+1}\right)}+\left[\widetilde{Q}_{i+1}\right] .
$$

The remaining equations,

$$
\Pi_{j, \bullet}^{\left(d_{i}-d_{j}\right)}=P_{j, i}^{(0)} Q_{i, \bullet}^{(0)}+\left(P_{j, i+1}^{(0)} \cdots P_{j, s}^{(0)}\right) Q_{i+1}^{\left(d_{i}-d_{i+1}\right)}+\left[\tilde{Q}_{i+1}\right]=0, \quad i+1 \leqslant j \leqslant s,
$$

can be rewritten as

$$
P_{i+1}^{(0)} Q_{i+1}^{\left(d_{i}-d_{i+1}\right)}=-\left(\begin{array}{c}
P_{i+1, i}^{(0)} \\
\vdots \\
P_{s, i}^{(0)}
\end{array}\right) Q_{i, \bullet}^{(0)}+\left[\widetilde{Q}_{i+1}\right], \quad \text { where } P_{i+1}^{(0)}=\left(\begin{array}{ccc}
P_{i+1, i+1}^{(0)} & \cdots & P_{i+1, s}^{(0)} \\
\vdots & \ddots & \vdots \\
P_{s, i+1}^{(0)} & \cdots & P_{s, s}^{(0)}
\end{array}\right)
$$

is invertible by the induction assumption. Put

$$
B=\left(\begin{array}{lll}
P_{i, i+1}^{(0)} & \cdots & P_{i, s}^{(0)}
\end{array}\right) \text { and } C=\left(\begin{array}{c}
P_{i+1, i}^{(0)} \\
\vdots \\
P_{s, i}^{(0)}
\end{array}\right) \quad \text { so that } P_{i}^{(0)}=\left(\begin{array}{cc}
P_{i, i}^{(0)} & B \\
C & P_{i+1}^{(0)}
\end{array}\right) \text {. }
$$

Thus, we get

$$
Q_{i+1}^{\left(d_{i}-d_{i+1}\right)}=-\left(P_{i+1}^{(0)}\right)^{-1} C Q_{i, \bullet}^{(0)}+X \widetilde{Q}_{i+1}=X^{\prime} \widetilde{Q}_{i}
$$


hence $Q_{i}^{(0)}$ is a left multiple of $\widetilde{Q}_{i}$. Moreover, substituting in (14), we get

$$
\Pi_{i, \bullet}^{(0)}=\left(P_{i, i}^{(0)}-B\left(P_{i+1}^{(0)}\right)^{-1} C\right) Q_{i, \bullet}^{(0)}+\left[\widetilde{Q}_{i+1}\right] .
$$

By assumption $\widetilde{Q}_{i+1}$ is in the row space of

$$
\left(\begin{array}{c}
\Pi_{i+1, \bullet}^{(0)} \\
\vdots \\
\Pi_{s, \bullet}^{(0)}
\end{array}\right)
$$

Hence $\operatorname{rk} \Pi_{i, \bullet}^{(0)}=n_{i}$ if and only if $\operatorname{rk}\left(P_{i, i}^{(0)}-B\left(P_{i+1}^{(0)}\right)^{-1} C\right) Q_{i, \bullet}^{(0)}=n_{i}$; that is, $\operatorname{det}\left(P_{i, i}^{(0)}-B\left(P_{i+1}^{(0)}\right)^{-1} C\right) \neq 0$. This matrix is the Schur complement of

$$
P_{i}^{(0)}=\left(\begin{array}{cc}
P_{i, i}^{(0)} & B \\
C & P_{i+1}^{(0)}
\end{array}\right),
$$

which is invertible exactly when $P_{i+1}^{(0)}$ is. Therefore (MC) is established in general, and by (15), $\widetilde{Q}_{i}$ is in the row span of $\widetilde{\Pi}_{i}$, so claim (A) is proved. Similarly, for a given $0 \leqslant m \leqslant d_{i-1}-d_{i}-1$, we can stack the remaining equations, (13), corresponding to $i \leqslant j \leqslant s$,

$$
\Pi_{j, \bullet}^{\left(d_{i}-d_{j}+m\right)}=\sum_{\ell=0}^{m} P_{j,(i: s)}^{(\ell)} Q_{i}^{\prime(m-\ell)}+\left[\widetilde{Q}_{i+1}\right]=0,
$$

to get the relation

$$
\left(\begin{array}{c}
\Pi_{i, \bullet}^{\left(d_{i}-d_{j}+m\right)} \\
\vdots \\
\Pi_{s, \bullet}^{\left(d_{i}-d_{j}+m\right)}
\end{array}\right)=\sum_{\ell=0}^{m} P_{i}^{(\ell)} Q_{i}^{(m-\ell)}=\left\{\begin{array}{cl}
\widetilde{\Pi}_{i} & \text { if } m=0 \\
0 & \text { otherwise }
\end{array}\right.
$$

Putting for notational simplicity $d=d_{i-1}-d_{i}$, we finally get

$$
\left(\begin{array}{cccc}
P_{i}^{(0)} & 0 & \cdots & 0 \\
P_{i}^{(1)} & P_{i}^{(0)} & & \vdots \\
\vdots & & \ddots & \vdots \\
P_{s}^{(d-1)} & \cdots & \cdots & P_{i}^{(0)}
\end{array}\right)\left(\begin{array}{c}
Q_{i}^{\prime(0)} \\
Q_{i}^{\prime(1)} \\
\vdots \\
Q_{i}^{(d-1)}
\end{array}\right)=\left(\begin{array}{c}
\Pi_{i}^{\prime} \\
0 \\
\vdots \\
0
\end{array}\right)+\left[\widetilde{Q}_{i+1}\right] \quad \text { where } \Pi_{i}^{\prime}=\left(\begin{array}{c}
\widetilde{\Pi}_{i} \\
0 \\
\vdots \\
0
\end{array}\right) .
$$

Accordingly, $Q_{i}^{(\ell)}$ is a left multiple of $Q_{i}^{(0)}$ for $0 \leqslant \ell \leqslant k-1$. Since we have established that $Q_{i}^{\prime(0)}$ is a left multiple of $\widetilde{Q}_{i}$, this ends the proof of claim (B). 
Proof of Proposition 38. Assume for simplicity that $D=\operatorname{diag}\left(d_{1} I_{n_{1}}, \ldots, d_{s} I_{n_{s}}\right)$ is written by blocks, and that $d_{1}>d_{2}>\cdots>d_{s}$. Then there exist $m=d_{1}-d_{s}$ matrices $T_{1}, \ldots, T_{m}$ of the type

$$
T_{i}=\left(\begin{array}{cc}
I_{b_{i}} & 0 \\
0 & 0_{n-b_{i}}
\end{array}\right),
$$

where every $b_{i}$ is equal to some $n_{1}+\cdots+n_{t_{i}}$ for some decreasing sequence $t_{i}$, such that $D=T_{1}+\cdots+T_{m}$. Secondly, assume that all left-upper square blocks of $P(0)$ of sizes $b_{i}$ are invertible. Letting $P=H_{0}$, according to Lemma 39, there exists a sequence of monopole matrices

$$
\Pi_{i}=\left(\begin{array}{cc}
I_{b_{i}} & t^{-1} \widetilde{\Pi}_{i} \\
0 & I_{n-b_{i}}
\end{array}\right)
$$

with a constant matrix $\widetilde{\Pi}_{i}$, and a sequence of lattice gauge transforms $H_{i} \in \mathrm{GL}_{n}(\mathrm{O})$ such that

$$
H_{i+1}=t^{-T_{i}} H_{i} t^{T_{i}} \Pi_{i}
$$

Let

$$
\bar{H}_{i}=t^{T_{i}} \Pi_{i}=\left(\begin{array}{cc}
t I_{b_{i}} & \widetilde{\Pi}_{i} \\
0 & I_{n-b_{i}}
\end{array}\right) .
$$

It follows from Lemma 40 that $\bar{H}=\bar{H}_{1} \cdots \bar{H}_{m}$ is strongly $D$-parabolic. It follows then, as a remarkable consequence, that the diagonal matrix $t^{D}$ can be both factored from the matrix $\bar{H}$ on the left as $\bar{H}=t^{D} \Pi$ with a monopole matrix $\Pi$, and simultaneously from the right as $\bar{H}=Q t^{D}$ with a lattice gauge $Q \in \mathrm{GL}_{n}(\mathcal{O})$. Since ${ }_{t}^{D} H_{d+1}=H \bar{H}$ holds, we get on the one hand that $t^{-D} P^{-1} t^{D} H_{m+1}=\Pi \in$ $\mathrm{GL}_{n}\left(\mathbb{C}\left[t^{-1}\right]\right)$ as required for the first claim of the permutation lemma. However, and this was not stated in [Bolibrukh 1990] or [Ilyashenko and Yakovenko 2007], we also have the relation $t^{D} \Pi=Q t^{D}$, which yields the second claim.

For the third claim, the sufficiency of the minors condition has just been established. For the converse, assume that we have the following diagram, which we have completed with a monopole $\Pi$ and a lattice gauge $Q$ such that $z^{-D} P z^{D} Q=\Pi$ :

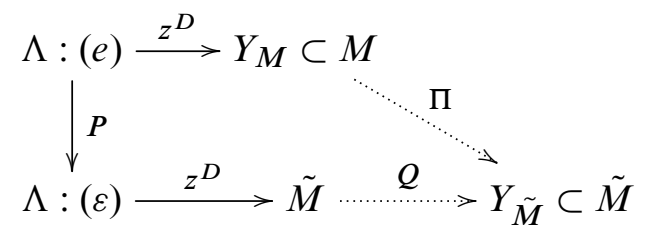

Let $Q=Q_{0}+Q_{1} z+\cdots \in \mathrm{GL}_{n}(\mathbb{O})$, and $\Pi=\Pi_{0}+\cdots+\Pi_{t} z^{-t} \in \mathrm{GL}_{n}\left(\mathbb{C}\left[z^{-1}\right]\right)$. Recall that if $\Pi$ is a monopole, then one must in particular have $\Pi_{0} \in \mathrm{GL}_{n}(\mathbb{C})$. Then 
Lemma 41 implies that such a factorization only exists if the matrix $P_{0}$ satisfies the condition on the minors.

The following scheme sums up this construction.
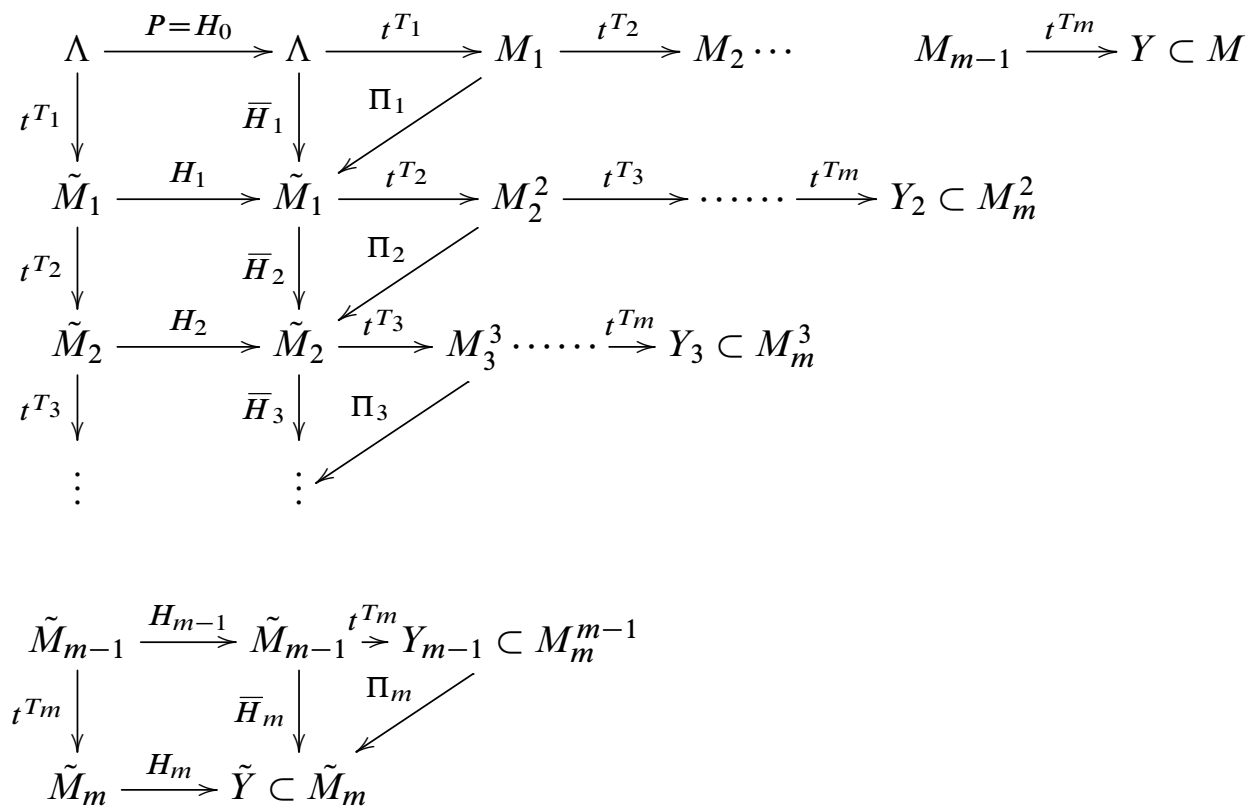

The first row corresponds to a min-shortest path $\Gamma=\left(\Lambda, M_{1}, \ldots, M\right)$ from $\Lambda$ to a given BG trivialization $M$. This path $\Gamma$ is included in an apartment $\mathscr{B}$, namely the one spanned by a BG basis ( $e$ ) of $\Lambda$ corresponding to the trivialization $M$. By definition, the apartment $\mathscr{B}$ goes through the global form $Y$ of $M$. The gauge $H^{-1}$ does not map the shortest path $\Gamma$ onto anything special. However, if we call $\mathscr{A}=H^{-1}(\mathscr{B})$ the image of the apartment spanned by $(e)$, the permutation lemma tells us how to construct a shortest path $\Gamma^{\prime}$ in $\mathscr{A}$ whose end point is also a BG trivialization of $\Lambda$. Lemma 39 gives the step-by-step modification of the shortest path $\Gamma$. Row $i$ of the diagram corresponds indeed to a partial shortest path $\Gamma_{i}=$ $\left(\tilde{M}_{i}, M_{i}^{i}, \ldots, M_{m}^{i}\right)$ whose end-point is a BG trivialization of the $i$-th element $\tilde{M}_{i}$ of the shortest path $\Gamma^{\prime}=\left(\Lambda, \tilde{M}_{1}, \ldots, \tilde{M}_{m}\right)$. Even if the end-point $\tilde{M}_{m}$ is a BG trivialization of $\Lambda$, note that the apartment $\mathscr{A}$ does not contain the global form $\tilde{Y}$ of $\tilde{M}_{m}$, and that we still need the gauge transform $H_{m}$ to obtain it.

3.3.1. Consequences of the permutation lemma. As stated in [Ilyashenko and Yakovenko 2007], one can assume that $\tau=$ id if all leading principal minors of $P$ are holomorphically invertible; that is, the corresponding minors of $P(0)$ are nonzero. This condition can always be ensured by a permutation of the columns of $P$. Actually, as stated in Proposition 38(3), it is sufficient that this condition 
holds only for the leading principal minors of orders $n_{1}, n_{1}+n_{2}, \ldots, n_{1}+\cdots+n_{s-1}$ of $P(0)$. We then say that $P$ respects the minors condition with respect to $D$ (or to $\left.\sigma=\left(n_{1}, \ldots, n_{s}\right)\right)$. Recall the following well-known result.

Lemma 42. Let $P \in \mathrm{GL}_{n}(\mathbb{C})$, let $(e)$ be the standard basis of $\mathbb{C}^{n}$ and $(\varepsilon)$ be the column vectors of $P$. Then $P$ respects the minors condition with respect to a signature $\sigma$ if and only if (e) is transversal to the flag $\boldsymbol{F}^{\sigma}(\varepsilon)$.

Definition 43. Let $\mathscr{A}$ be an apartment induced by the $K$-frame $\Phi$ in $V$. Consider $\Lambda \in A$. Let $\boldsymbol{H}$ be the Harder-Narasimhan flag of $\Lambda$ in $E=\Lambda / \mathfrak{m} \Lambda$. Let $W \subset S_{n}$ be the parabolic subgroup of $S_{n}$ associated to $\boldsymbol{H}$, and $W^{\prime}$ be the set of right cosets $W \backslash S_{n}$. Let $(e)$ be a basis of $\Lambda$ in the frame $\Phi$ whose image $(\bar{e})$ in $E$ is transversal to $\boldsymbol{H}$. The integer

$$
\iota_{\Lambda}(\mathscr{A})=\mid\left\{\tau \in W^{\prime} \mid \tau(\bar{e}) \text { transversal to } \boldsymbol{H}\right\} \mid
$$

is independent of (e) and is called the transversality index of $\mathscr{A}$ with respect to $\Lambda$.

Theorem 44. Let $\mathscr{E}$ be a holomorphic vector bundle over $X$, and let $\Lambda=\mathscr{E}_{x} \in \mathbf{\Lambda}$ be its stalk at $x \in X$.

(i) For any apartment $\mathscr{A}$ in the Bruhat-Tits building $B$ at $x$ such that $[\Lambda] \in \mathscr{A}$, there exists a $B G$ trivialization of $\Lambda$ in $A$.

(ii) More precisely, the number of $B G$ trivializations of $\Lambda$ in $\mathscr{A}$ is exactly

$$
|\mathscr{A} \cap \operatorname{BG}(\Lambda)|=\iota_{\Lambda}(\mathscr{A}) .
$$

Proof. Let (e) be a BG basis of $\Lambda$, and $M \in \mathrm{BG}(\Lambda)$. Let $(\varepsilon)$ be a basis of the lattice $\Lambda$ which spans the apartment $\mathscr{A}$. Since $\mathscr{A}$ is invariant under $S_{n}$, we can assume that the matrix $P \in \mathrm{GL}_{n}(\mathcal{O})$ of the basis change from $(\varepsilon)$ to $(e)$ has invertible principal leading minors. According to the permutation lemma, there exists a matrix $\tilde{P} \in \mathrm{GL}_{n}(\mathrm{O})$ such that

$$
\Pi=z^{-D} P^{-1} z^{D} \tilde{P} \in \mathrm{GL}_{n}\left(\mathbb{C}\left[z^{-1}\right]\right) .
$$

The gauge $\Pi$ sends the basis of global sections $(\sigma)=\left(z^{D} e\right)$ of the BG trivialization of $\mathscr{E}$, given at $x$ by $M$, into a basis $(\tilde{e})$ of $\tilde{M}$. Since $\Pi$ is a monopole, the basis $(\tilde{e})$ is also a global basis of sections, but spans another trivializing bundle, namely $\mathscr{F}=\mathscr{E} \tilde{M}$. Therefore the arbitrary apartment $\mathscr{A}$ spanned by $(\varepsilon)$ indeed contains a trivial bundle. Now the matrix $\bar{H}=z^{D} \Pi$ admits a right factorization $\bar{H}=Q z^{D}$. As a consequence, if we let $(\widetilde{\varepsilon})$ be the basis of $\Lambda$ obtained from $(e)$ by the matrix $Q$, then $z^{D}(\widetilde{\varepsilon})$ is also a basis of $Y_{\tilde{M}}$. The following scheme sums up the situation. 


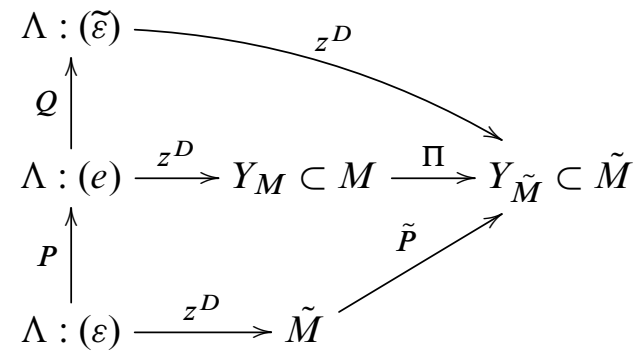

Therefore the lattice $\tilde{M}$ is also a BG trivialization of $\Lambda$.

The monopole gauge $\Pi$ is block-upper-triangular according to $D$, and its block matrices $\Pi_{i j}$ satisfy

$$
d_{j}-d_{i} \leqslant v\left(\Pi_{i j}\right) \leqslant \operatorname{deg} \Pi_{i j} \leqslant 0 .
$$

This means that $\Pi$ respects the Harder-Narasimhan filtration $\mathrm{HN}_{\Lambda}$ of $V$ corresponding to the lattice $\Lambda$. Conversely, the lattice gauge $Q$ has a lower $D$-block-triangular constant term $Q_{0}$. Since $z^{-D} P Q z^{D} \in \mathrm{GL}_{n}(\mathcal{O})$, the matrix $P Q$ has an upper $D$ block-triangular constant term $P_{0} Q_{0}$. Therefore, if we put $P_{D}, P_{D}^{-}$for the pair of opposite $D$-parabolic standard subgroups of $\mathrm{GL}_{n}(\mathbb{C})$, the matrix $P_{0}$ satisfies $P_{0} \in P_{D}^{-} P_{D}$. This means exactly that $P$ satisfies the minors condition with respect to $D$. If we permute the vectors of $(\varepsilon)$ with $\tau \in S_{n}$ in such a way that $D_{\tau^{-1}} \neq D$, the permutation lemma ensures that $\mathscr{L}\left(z^{D}\left(\varepsilon_{\tau}\right)\right) \neq \tilde{M}$ is again a BG trivialization of $\Lambda$. This establishes the second claim of the theorem.

Corollary 45. For any apartment $\mathscr{A} \ni \Lambda$, there exists an ordered basis $(\varepsilon)$ of $\mathscr{A}$ and a $B G$ basis (e) of $\Lambda$ such that the gauge $P$ from $(e)$ to $(\varepsilon)$ has a lower block$D$-triangular unipotent constant term $P_{0}$, and that the following picture holds.

$$
\begin{array}{cc}
\Lambda:(\varepsilon) \stackrel{z^{D}}{\longrightarrow} M \\
P=P_{0}+z \tilde{U} \uparrow & \uparrow \\
\Lambda:(e) \stackrel{z^{D}}{\longrightarrow} Y_{M}
\end{array}
$$

The number of BG trivializations in $\mathscr{A}$ can hence be computed from a matrix $P_{0} \in \mathrm{GL}_{n}(\mathbb{C})$ with a simple structure:

$$
P_{0}=\left(\begin{array}{ccc}
I_{n_{1}} & \cdots & 0 \\
\vdots & \ddots & \vdots \\
X_{i j} & \cdots & I_{n_{s}}
\end{array}\right)
$$

as the number of permutations not leaving $D$ invariant whose action on the columns of $P_{0}$ preserve the minors condition. The following result completes the proof of Theorem 25. 
Corollary 46. Let $\Lambda \in \Lambda$ and $T=T(\Lambda)$.

(i) For any basis $(\varepsilon)$ of $\Lambda$, let $\tilde{M}=\mathscr{L}\left(z^{T}(\varepsilon)\right)$. Then either $\tilde{M} \in \operatorname{BG}(\Lambda)$ or $\tilde{M} \notin \Lambda^{0}$.

(ii) For any flag $\boldsymbol{F}^{\prime}$ in $\Lambda / \mathfrak{m} \Lambda$ transversal to $\mathrm{HN}_{\Lambda}$, and any form $Y$ in $\Lambda$, the lattice $\tilde{M}=\mathscr{L}_{Y}\left(\boldsymbol{F}^{\prime}, T\right)$ is a $B G$ trivialization of $\Lambda$.

In particular, if $M \in \mathrm{BG}(\Lambda)$, then $\tilde{M}=\mathscr{L}_{Y}\left(\boldsymbol{F}^{\Lambda}(M), T\right) \in \mathrm{BG}(\Lambda)$ for any form $Y$ in $\Lambda$.

Proof. For (i), if $\tilde{M}$ is trivial, then, by Lemma 41, the gauge from (e) to $(\varepsilon)$ satisfies the minors condition; hence $\tilde{M}$ is BG. According to Lemma 27, there exists $M \in \operatorname{BG}(\Lambda)$ such that $\boldsymbol{F}^{\prime}=\boldsymbol{F}^{\Lambda}(M)$. Let $(e)$ be a Smith basis of $\Lambda$ for $M$. The lattice $\tilde{M}$ is spanned by $z^{T}\left(e_{Y}\right)$ where $\left(e_{Y}\right)$ is the $Y$-basis of $(e)$. The gauge $P$ from $(e)$ to $\left(e_{Y}\right)$ has invertible principal minors, since it is tangent to $I$. Item (ii) follows.

The permutation lemma is a sort of converse to the Birkhoff-Grothendieck theorem. It can also be seen as a lifting and factorization lemma. For a standard parabolic subgroup $P \subset \mathrm{GL}_{n}(\mathbb{C})$, let

$$
\mathbb{Z}_{P}^{n}=\left\{D \in \mathbb{Z}^{n} \mid d_{i}<d_{j} \Rightarrow A_{i j}=0 \text { for all } A \in P\right\} .
$$

Corollary 47. Let $A \in \mathrm{GL}_{n}(\mathcal{O})$. Let $P, P^{-}$be a pair of opposed standard parabolic subgroups of $\mathrm{GL}_{n}(\mathbb{C})$. Then we have, for $w \in S_{n}$,

$$
A_{0} \in P w P^{-} \Leftrightarrow\left\{\begin{array}{c}
\forall D \in \mathbb{Z}_{P}^{n}, \exists \Pi \in \mathrm{GL}_{n}\left(\mathbb{C}\left[z^{-1}\right]\right), \exists B \in \mathrm{GL}_{n}(\mathbb{O}) \\
\text { such that } A=z^{D} \Pi B z^{-w D}
\end{array}\right.
$$

Moreover, if this holds, then $B \in \mathscr{G}_{-D}$ and $\Pi \in \hat{P}$ hold, where $\hat{P}$ stands here for the subgroup of upper-D-triangular matrices of $\mathrm{GL}_{n}\left(\mathbb{C}\left[z^{-1}\right]\right)$.

This result means that there is a cell decomposition of $\mathrm{GL}_{n}(\mathrm{O})$ whose cells (defined by the right-hand side of the previous relation) are mapped surjectively (by the canonical surjection $\mathrm{GL}_{n}(\mathbb{O}) \rightarrow \mathrm{GL}_{n}(\mathbb{C})$ ) on the Schubert-Bruhat cells of $\mathrm{GL}_{n}(\mathbb{C})$. Finally, a last consequence of Proposition 38 is that the lattices involved in the Birkhoff-Grothendieck algorithm given in Section 3.2.1 can be taken inside a single apartment.

Corollary 48. Let $\Lambda \in \Lambda$. For any $M \in \Lambda^{0}$, let $\Gamma(M, \Lambda)=\left(\Lambda_{0}=M, \ldots, \Lambda_{t}=\Lambda\right)$ be the min-shortest path from $M$ to $\Lambda$. For any apartment $\mathscr{A} \ni \Lambda, M$, there exist $\tilde{M}_{1}, \ldots, \tilde{M}_{t} \in \mathscr{A} \cap \boldsymbol{\Lambda}^{0}$ such that $\tilde{M}_{i} \in \operatorname{BG}\left(\Lambda_{i}\right)$ for $1 \leqslant i \leqslant t$.

Proof. Since $\mathscr{A} \ni \Lambda, M$, we have $\Lambda_{i} \in \mathscr{A}$ for $1 \leqslant i \leqslant t$. By Theorem 44, any lattice in $\mathscr{A}$ admits a BG trivialization in $\mathscr{A}$. 


\section{Local meromorphic connections}

Let $\operatorname{Der}_{\mathbb{C}}(K)$ be the $K$-vector space of dimension one of $\mathbb{C}$-derivations of $K$ and $\Omega=\Omega_{\mathbb{C}}^{1}(K)$ the dual composed of differentials of $K$. The valuation $v$ extends naturally to these spaces by the formulæ $v(\vartheta)=v(f)$ and $v(\omega)=v(g)$ if $\vartheta=$ $f d / d z$ and $\omega=g d z$ for any uniformizing parameter $z$ of $K$. The space $\Omega$ is naturally filtered by the rank-one free 0 -modules $\Omega(k)=\{\omega \in \Omega \mid v(\omega) \geqslant-k\}$.

Let $V$ be a $K$-vector space of finite dimension $n$ and let $\Omega(V)=V \otimes_{K} \Omega_{\mathbb{C}}^{1}(K)$. We fix a meromorphic connection $\nabla$ on $V$. This is an additive map $\nabla: V \rightarrow \Omega(V)$ satisfying the Leibniz rule

$$
\nabla(f v)=v \otimes d f+f \nabla v \quad \text { for all } f \in K \text { and all } v \in V .
$$

For any basis $(e)=\left(e_{1}, \ldots, e_{n}\right)$ of $V$, the matrix $\operatorname{Mat}(\nabla,(e))$ of the connection $\nabla$ in the basis $(e)$ is the matrix $A=\left(A_{i j}\right) \in \mathrm{M}_{n}(\Omega)$ such that

$$
\nabla e_{j}=-\sum_{i=1}^{n} e_{i} \otimes A_{i j} \quad \text { for all } j=1, \ldots, n .
$$

If the matrix $P=\operatorname{Mat}\left(\mathrm{id}_{V},(\varepsilon),(e)\right) \in \mathrm{GL}_{n}(K)$ is the basis change from (e) to any other basis $(\varepsilon)$, then the matrix of $\nabla$ in $(\varepsilon)$ is given by the gauge transform of $A$ :

$$
A_{[P]}=P^{-1} A P-P^{-1} d P .
$$

For any derivation $\tau \in \operatorname{Der}(K / \mathbb{C})$, the contraction of $\nabla$ with $\tau$ induces a differential operator $\nabla_{\theta}$ on $V$. The connection $\nabla$ is regular whenever the set of logarithmic lattices

$$
\Lambda_{\log }=\left\{\Lambda \in \boldsymbol{\Lambda} \mid \nabla(\Lambda) \subset \Lambda \otimes_{\mathbb{O}} \Omega(1)\right\}
$$

is nonempty. For any logarithmic lattice $\Lambda \in \Lambda_{\log }$, the connection $\nabla$ induces a well-defined residue endomorphism $\operatorname{Res} \Lambda \nabla \in \operatorname{End}_{\mathbb{C}}(\Lambda / \mathfrak{m} \Lambda)$. Note that, since the set $\boldsymbol{\Lambda}_{\log }$ is closed under homothety and module sums and intersections [Corel 2004, Lemma 2.5], it induces a path-convex subset of the Bruhat-Tits building: if $L, L^{\prime} \in \Lambda_{\log }$, then every shortest path between $L$ and $L^{\prime}$ is a subset of $\boldsymbol{\Lambda}_{\log }$. This applies in particular to both $\Gamma_{\max }\left(L, L^{\prime}\right)$ and $\Gamma_{\min }\left(L, L^{\prime}\right)$.

4.1. The Deligne lattice. As is well known, the choice of a matrix logarithm of the monodromy corresponds to fixing a special lattice in the space $V$. More precisely, let $V^{\nabla} \subset V \otimes_{K} H$ be the $\mathbb{C}$-vector space of horizontal sections on any PicardVessiot extension $H$ of $K$. Let $g=g_{s} g_{u} \in \operatorname{End}\left(V^{\nabla}\right)$ be the multiplicative Jordan decomposition of the corresponding local monodromy map. Then the logarithm of the unipotent part $g_{u}$ is canonically defined (by the Taylor expansion formula for $\log (1+x)$ ), but there are several ways to define the logarithm of the semisimple 
part $g_{s}$. Namely, one must fix a branch of the complex logarithm for every distinct eigenvalue of $g_{s}$.

A well known result (variously attributed to Deligne, Manin, ... ) says that this choice uniquely defines a lattice in $V$. In Deligne's terms, for any section $\sigma$ of $\mathbb{C} \rightarrow \mathbb{C} / \mathbb{Z}$, there is a unique logarithmic lattice $\Delta_{\sigma}$ such that the eigenvalues of the residue map $\operatorname{Res}_{\Delta_{\sigma}} \nabla$ are in the image $\operatorname{Im} \sigma$ of $\sigma$. As a habit, one usually takes $\operatorname{Re}(\operatorname{Im} \sigma) \subset[0,1[$. In fact, such a habit is not as arbitrary as it seems.

Proposition 49. Assume that the connection $\nabla$ admits an apparent singularity (i.e., the monodromy map is trivial). Then the matrix $\operatorname{Mat}(\nabla,(e))$ is holomorphic if and only if the lattice spanned by $(e)$ is equal to the Deligne lattice $\Delta$ attached to $\operatorname{Re}(\operatorname{Im} \sigma) \subset[0,1[$.

Proof. Since the monodromy map is trivial, its normalized logarithm with respect to $\Delta$ is 0 . Hence, there is a basis of $\Delta$ where the connection has matrix 0 . In any other basis (e) of $\Delta$, the connection has matrix $A=P^{-1} d P$ with $P \in \mathrm{GL}_{n}(\mathrm{O})$, which is holomorphic. Let $M$ be another lattice, and let (e) be a Smith basis of $\Delta$ for $M$. Then the matrix in a basis of $M$ is given by the gauge equation

$$
\tilde{A}=z^{-D} A z^{D}-z^{-D} d\left(z^{D}\right)=\left(A_{i j} z^{k_{j}-k_{i}}\right)-D \frac{d z}{z} .
$$

The nonzero diagonal terms of the matrix $D$ of elementary divisors of $M$ give necessarily rise to a pole of order 1 in $\widetilde{A}$. Therefore, $\Delta$ is the only lattice where the connection has a holomorphic matrix.

As a result, we will call $\Delta$ the Deligne lattice of $V$.

4.1.1. Birkhoff forms. According to a very classical result (see, e.g., [Gantmacher 1959, p. 150]), if

$$
\Omega=\operatorname{Mat}(\nabla,(e))=\sum_{k \geqslant 0} A_{k} z^{k} \frac{d z}{z}
$$

is the series expansion in $z$ of the matrix of $\nabla$ in a basis $(e)$ of $\Delta$, the gauge $P=\sum_{k \geqslant 0} P_{k} z^{k} \in \mathrm{GL}_{n}(\mathcal{O})$ defined recursively by

$$
\left\{\begin{array}{l}
P_{0}=I, \\
P_{k}=\Phi_{A_{0}, A_{0}-k I}^{-1}\left(Q_{k}\right), \quad \text { where } Q_{k}=\sum_{i=1}^{k} A_{i} P_{k-i},
\end{array}\right.
$$

transforms $\Omega$ into $A_{0} d z / z$. Here we put $\Phi_{U, V}(X)=X U-V X$. Recall that the map $\Phi_{U, V}$ is an automorphism of $\mathfrak{g l}(\mathbb{C})$ when the spectra of $U$ and $V$ are disjoint. The gauge $P$ thus defined is uniquely determined; moreover, the set of bases where $\nabla$ has matrix $L \frac{d z}{z}$ where $L \in \mathrm{M}_{n}(\mathbb{C})$ is a constant matrix spans a form $\Upsilon_{z}$ of $\Delta$, that we call the Birkhoff form of the Deligne lattice $\Delta$. The gauge transform $P$ sends in fact the basis $(e)$ to its $\Upsilon_{z}$-basis, that we denote here for simplicity by $\left(e_{z}\right)$. 
As it results from the proof of Proposition 49, when the singularity is apparent, the Birkhoff form is uniquely defined. Otherwise, however, the form $\Upsilon_{z}$ depends on the choice of the local coordinate $z$. Two Birkhoff forms are nevertheless canonically isomorphic.

Lemma 50. Let $z$, $t$ be two local coordinates, and let $\alpha \in 0^{*}$ such that $z=\alpha t$. Let $P_{z}$ and $P_{t}$ be the gauge transforms that send $(e)$ to $\left(e_{z}\right)$ and $\left(e_{t}\right)$, respectively. There is a unique gauge transform $\widetilde{P}$ that sends $\left(e_{z}\right)$ to $\left(e_{t}\right)$.

Proof. One has $\frac{d z}{z}=u \frac{d t}{t}$ with $u=1+\frac{\theta_{t} \alpha}{\alpha}$ where $\theta_{t}=t \frac{d}{d t}$. Put $u=\sum_{i=0}^{\infty} u_{i} t^{i}$. Accordingly, the matrix of the connection in $\left(e_{z}\right)$ satisfies

$$
\operatorname{Mat}\left(\nabla,\left(e_{z}\right)\right)=A_{0} \frac{d z}{z}=A_{0}\left(\sum_{i=0}^{\infty} u_{i} t^{i}\right) \frac{d t}{t} .
$$

There exists therefore a uniquely defined gauge transform $\widetilde{P}=\sum_{i=0}^{\infty} \widetilde{P}_{i} t^{i}$ that transforms the expression $A_{0} d z / z$ into $A_{0} d t / t$, as explained in the following scheme.

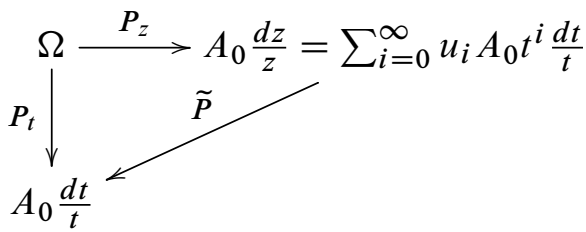

The matrix series $\widetilde{P}$ is determined recursively by the equations (18) applied to the series $\sum_{i=0}^{\infty} A_{0} u_{i} t^{i}$. The coefficients $\widetilde{P}_{i}$ are even polynomials in $A_{0}$, defined by the induction rule

$$
\widetilde{P}_{0}=I, \quad \widetilde{P}_{k}=\frac{1}{k} \sum_{i=1}^{k} u_{i} A_{0} \widetilde{P}_{k-i}
$$

4.2. Logarithmic lattices and stable flags. When two lattices $\Lambda, M$ are adjacent, all the relevant information on $M$ can be retrieved from the quotient $M / \mathfrak{m} \Lambda$. This is also true in presence of a connection.

Lemma 51. Let $\Lambda \in \Lambda_{\log }$ be a logarithmic lattice. For any adjacent lattice $M \in$ $[\mathfrak{m} \Lambda, \Lambda]$, we have $M \in \Lambda_{\log }$ if and only if $M / \mathfrak{m} \Lambda$ is $\operatorname{Res}_{\Lambda} \nabla$-stable.

Proof. In any basis (e) of $\Lambda$ such that the images of the first $m=\operatorname{dim} W$ vectors span $W=M / \mathfrak{m} \Lambda$, the connection matrix $\Omega=\operatorname{Mat}(\nabla,(e))$ has a residue of the form $\left(\begin{array}{ll}A & B \\ 0 & C\end{array}\right) \in \mathrm{M}_{n}(\mathbb{C})$, where $A \in \mathrm{M}_{m}(\mathbb{C})$. Putting $T=\operatorname{diag}\left(0_{m}, I_{n-m}\right)$, the basis $(\varepsilon)=z^{T}(e)$ spans $M$. It is then straightforward that the matrix $z^{-T} \Omega z^{T}-T \frac{d z}{z}$ of $\nabla$ in $(\varepsilon)$ has a simple pole.

When the lattices are further apart, this correspondence fails. However, there is also a complete description of the logarithmic lattices as follows. Let $\Delta$ be 
the Deligne lattice, and let $\delta_{\Delta}=\operatorname{Res}_{\Delta} \nabla$ be the residue $\mathbb{C}$-endomorphism on $\mathbf{D}=\Delta / \mathfrak{m} \Delta$. Let $\Upsilon$ be the Birkhoff form of $\Delta$ attached to a uniformizing parameter $z$. Logarithmic lattices can then be characterized as stable flags (as already remarked in [Sabbah 2002, Theorem III.1.1]).

Proposition 52. The set $\boldsymbol{\Lambda}_{\log }$ of logarithmic lattices is in bijection with the subset $\Xi_{0}(\Upsilon)$ of filtrations of $\Upsilon$ which are stable under the residue, namely

$$
\Xi_{0}(\Upsilon)=\left\{(\boldsymbol{F}, D) \in \Xi(\Upsilon) \mid \boldsymbol{F} \in \mathfrak{F l}_{\delta_{\Delta}}\right\} .
$$

Proof. According to a classical, although not so well known, result (which can be found for instance in [Babbitt and Varadarajan 1983; Bolibrukh 1990]), a lattice $\Lambda \in \Lambda$ is logarithmic if and only if

(i) there exists a basis $(e)$ of $\Upsilon$ such that $\left(z^{D} e\right)$ is a basis of $\Lambda$, with $D=\mathbf{E D}_{\Delta}(\Lambda)$, (ii) $z^{-D} L z^{D} \in \mathrm{M}_{n}(\mathcal{O})$, where $L=\operatorname{Mat}\left(\nabla_{z \frac{d}{d z}},(e)\right)$.

It results from (ii) that in this case, the matrix $L$ is $D$-parabolic. Since the flag $\boldsymbol{F}^{\Delta}(\Lambda)$ induced by $\Lambda$ on $\mathbf{D}=\Delta / \mathfrak{m} \Delta$ is spanned by the images of the basis (e) in $\mathbf{D}$, it is stable under $\delta_{\Delta}$. Conversely, it is simply a matter of computation to show that any lattice in the $\Upsilon$-fiber of a $\delta_{\Delta}$-stable flag of $\mathbf{D}$ is logarithmic.

A difference between our result and Sabbah's is that he only states this result as an equivalence of categories between the set of stable filtrations of $\mathbf{D}$ and the logarithmic lattices, whereas we give the explicit correspondence based on the lifting of $\mathbf{D}$ to a Birkhoff form. Although it would seem that the previous result has little value to effectively determine all logarithmic lattices, it is always possible to determine them in finite terms.

Lemma 53. Let $M \in \boldsymbol{\Lambda}_{\log }$ and let $(\boldsymbol{F}, D)=\Pi_{\Delta}(M)$. Let $Y$ be a form of $\Delta$, and let (e) be a basis of $Y$ respecting the flag $\boldsymbol{F}$. Fix a coordinate $z$, and let $P=I+P_{1} z+\cdots$ be the gauge from (e) to its $\Upsilon_{z}$-basis $\left(e_{z}\right)$. Then the Laurent polynomial gauge $Q \in \mathfrak{g l}\left(\mathbb{C}\left[z, z^{-1}\right]\right)$ defined by

$$
Q=\left(I+\cdots+P_{d-1} z^{d-1}\right) z^{D}, \quad \text { where } d=d(\Delta, M),
$$

sends the basis (e) of $\Delta$ to a basis of $M$.

Proof. This is an almost direct consequence of Lemma 15.

Note that the polynomial gauge $Q$ can be explicitly computed from formula (18). On the other hand, one can also explicitly describe the set $\Xi_{0}(\Upsilon)$. For a linear map $f \in \operatorname{End}\left(\mathbb{C}^{n}\right)$, say that an apartment $\mathscr{A}_{\Phi}$ is a diagonalizing apartment of $f$ if the frame $\Phi$ is composed of eigenlines of $f$. 
Lemma 54. Let $\delta_{\Delta}=\mathfrak{d}+\mathfrak{n}$ be the additive Jordan decomposition of the residue map $\delta_{\Delta}=\operatorname{Res}_{\Delta} \nabla$. The pair $(\boldsymbol{F}, D) \in \Xi(\Upsilon)$ is an element of $\Xi_{0}(\Upsilon)$ if and only if $\boldsymbol{F}$ admits a complete flag refinement $\hat{\boldsymbol{F}}$ such $\hat{\boldsymbol{F}} \in \mathfrak{F l}_{\mathfrak{n}}$ and there is a diagonalizing apartment $A$ for $\mathfrak{d}$ that respects the flag $\hat{\boldsymbol{F}}$.

Proof. $\boldsymbol{F}$ is $\delta$-stable if and only if it is stable under both $\mathfrak{d}$ and $\mathfrak{n}$. It is known that $\boldsymbol{F}$ is stable under $\mathfrak{d}$ if and only if every component $F_{i}$ of $\boldsymbol{F}$ is a direct sum of $\mathfrak{d}$-stable lines, and under $\mathfrak{n}$ if and only if it admits a complete flag refinement $\hat{\boldsymbol{F}} \in \mathfrak{F l}_{\mathfrak{n}}$.

\section{The Riemann-Hilbert problem}

This problem is by now very well-known, so we will just state the necessary notations and definitions, and refer to the classical paper of Bolibrukh [1990] and to the account he gives of the construction of the Deligne bundle (see [Sabbah 2002; Ilyashenko and Yakovenko 2007] and also [André and Baldassarri 2001] for a purely algebraic construction).

Let $\mathscr{S}=\left\{s_{1}, \ldots, s_{p}\right\}$ be a prescribed set of singular points, $z_{0} \notin \mathscr{Y}$ be an arbitrary base point, and let $\chi$ denote a representation

$$
\chi: \pi_{1}\left(X \backslash \mathscr{Y}, z_{0}\right) \rightarrow \mathrm{GL}_{n}(\mathbb{C}) .
$$

The Riemann-Hilbert problem asks informally for a linear differential system having $\chi$ as monodromy representation. In the terms used in this paper, it asks for a regular meromorphic connection $\nabla$ with singular set $\mathscr{Y}$ and monodromy $\chi$ on a holomorphic vector bundle $\mathscr{E}$. If the bundle is required to be logarithmic with respect to $\nabla$ one speaks of a weak solution to RH. In its strongest form, the Riemann-Hilbert problem asks for a differential system $Y^{\prime}=A(z) Y$ having simple poles on $\mathscr{S}$ as only singularities, and whose monodromy representation is globally conjugate to $\chi$. This amounts to asking for a weak solution $(\mathscr{E}, \nabla)$ which is moreover trivial.

5.1. The Röhrl-Deligne construction. We briefly recall H. Röhrl's construction (as presented, for instance, in [Bolibrukh 1990; Bolibrukh et al. 2006]). Let $\mathfrak{U}=\left(U_{i}\right)_{i \in I}$ be a finite open cover of $X^{*}=X \backslash \mathscr{Y}$ by connected and simply connected open subsets $U_{i} \subset X^{*}$ such that their intersection has the same property, and all triple intersections are empty. Consider arbitrary points $z_{i} \in U_{i}$ and $z_{i j} \in U_{i} \cap U_{j}$, and paths $\gamma_{i}: z_{0} \rightarrow z_{i}$ and $\gamma_{i j}: z_{i} \rightarrow z_{i j}$, so that $\delta_{i j}=\gamma_{i} \gamma_{i j} \gamma_{j i}^{-1} \gamma_{j}^{-1}$ is a positively oriented loop around $z_{i}$ having winding number 1 . Then the cocycle $g=\left(g_{i j}\right)$ defined over $\mathfrak{U}$ by the constant functions $g_{i j}=\chi\left(\left[\delta_{i j}\right]\right)$ defines a flat vector bundle $\mathscr{F}$ over $X^{*}$. Define the connection $\nabla$ over $U_{i}$ by the (0) matrix in the basis of sections corresponding to the cocycle $g$. The $\nabla$-horizontal sections of $\mathscr{F}$ have by construction the prescribed monodromy behavior. This solves what we called the topological Riemann-Hilbert problem in our introduction. 
Now add a small neighborhood $D$ of each singular point $s \in \mathscr{Y}$ to the cover $\mathfrak{U}$, in such a way that $D \backslash\{s\}$ is covered by $k$ pairwise overlapping sectors $\Sigma_{1}=D \cap U_{j_{1}}$, $\ldots, \Sigma_{k}=D \cap U_{j_{k}}$. On an arbitrarily chosen sector among the $\Sigma_{i}$, say, $\Sigma_{1}$, let $\tilde{g}_{s 1}=z^{L}$ where $z$ is a local coordinate at $s$ and

$$
L=\frac{1}{2 i \pi} \log \chi(\delta),
$$

normalized with eigenvalues having their real part in the interval [0, 1[. Since the open subset $\Sigma_{1}$ only intersects $\Sigma_{2}$ and $\Sigma_{k}$, the only necessary cocycle relations to satisfy are $\tilde{g}_{s 2}=\tilde{g}_{s 1} g_{12}$ and $\tilde{g}_{s k}=\tilde{g}_{s 1} g_{1 k}$, which we take as definition of the cocycle elements $\tilde{g}_{s 2}$ and $\tilde{g}_{s k}$. Define in this way the remaining elements of the cocycle $\tilde{g}$ on $D \cap U_{j_{i}}$. By construction, the result defines a holomorphic vector bundle $\mathfrak{D}$ on the whole of $X$, and the connection $\nabla$ can be extended as $L \frac{d z}{z}$ in the basis of sections $(\sigma)$ of $\mathfrak{D}$ over $D$ chosen to construct $\tilde{g}_{s 1}$. The pair $(\mathfrak{D}, \nabla)$ is called the Deligne bundle of $\chi$. This construction solves simultaneously the meromorphic and the weak Riemann-Hilbert problem.

Note 55. The basis $(\sigma)$ is, in our terms, a basis of the Birkhoff form attached to the coordinate $z$ at $s$.

5.2. Weak and strong solutions. The Riemann-Hilbert problem can be seen as involving three different levels. The topological level is only governed by the (analytic) monodromy around the prescribed singular set. The meromorphic level is essentially based on the solution of the local inverse problem. The third one, that we call holomorphic is global and asks for the existence of a trivial holomorphic vector bundle. In fact, separating these three aspects is not so easy to do, because the Röhrl-Deligne construction in fact yields a particular holomorphic vector bundle $\mathscr{E}$ with a connection $\nabla$ that already respects the holomorphic prescribed behavior.

What makes the strong Riemann-Hilbert problem a difficult one is precisely this third level. The local meromorphic invariants added to the topological solution of the inverse monodromy specify up to meromorphic equivalence class the connection $\nabla$ on $X$. In this respect, the natural category to state this construction is not the category of holomorphic vector bundles with meromorphic connections, but the meromorphic vector bundles, that is, pairs $(\mathscr{V}, \nabla)$ where $\mathscr{V}$ is locally (but in fact globally) isomorphic to $\mu_{X}^{n}$. This is why we call the second step meromorphic. The Riemann-Hilbert problem with the given data solved here corresponds to the very weak Riemann-Hilbert problem (as coined in [Sabbah 2002]): any subsheaf $\mathscr{F}_{F}$ of locally free $0_{X}$-modules contained in the (trivial) meromorphic bundle $\mathscr{V}$ is endowed naturally with the connection $\nabla$, and therefore is a holomorphic vector bundle with a regular connection having the prescribed monodromy. As stated by the next result (and otherwise well known), all solutions to the weak problem are obtained as local modifications of the Deligne bundle. 
Proposition 56. Let $\tilde{\pi}: \widetilde{E} \rightarrow X$ and $\tilde{\nabla}: \widetilde{\mathscr{E}} \rightarrow \widetilde{\mathscr{E}} \otimes_{\mathcal{O}} \Omega$ be a weak solution to the Riemann-Hilbert problem. Then there exist a finite set $S \subset X$, and local lattices $M_{x}$ for $x \in S$ such that the pair $(\widetilde{\mathscr{E}}, \widetilde{\nabla})$ is holomorphically isomorphic to $\left(\mathfrak{D}^{M}, \nabla\right)$.

The last step of the strong Riemann-Hilbert problem consists of searching the set of holomorphic vector bundles endowed with the connection $\nabla$ for a bundle which at the same time has the required holomorphic invariants and is holomorphically trivial. A negative answer requires to know all the holomorphic vector bundles with this prescribed logarithmic property. Note that up to this point, the discussion presented in this section holds over an arbitrary compact Riemann surface.

5.2.1. Plemelj's theorem. In 1908, the Slovenian mathematician J. Plemelj (see [Plemelj 1964]) proved a first version of the strong Riemann-Hilbert problem, under the assumption that at least one monodromy is diagonalizable. Whereas his first proof used an analytic approach (Fredholm integrals) to construct the actual matrix of solutions, to thence deduce the differential system and prove that it has only simple poles, the general framework of vector bundles recalled so far allows to establish this fact in an amazingly concise way.

Theorem 57 (Plemelj). If one of the elementary monodromy maps from representation $\chi: \pi_{1}\left(X \backslash \mathscr{Y}, z_{0}\right) \rightarrow \mathrm{GL}_{n}(\mathbb{C})$ is diagonalizable, then the Riemann-Hilbert problem has a strong solution.

Proof. Let $(\mathfrak{D}, \nabla)$ be the Röhrl-Deligne bundle attached to the representation $\chi$. Let, say $G=\chi(\gamma)$ around $s \in \mathscr{Y}$, be diagonalizable. Let $\Upsilon$ be a Birkhoff form at $s$, and let (e) be a basis of $\Upsilon$ where $G$ is diagonal. According to condition (ii) in Section 4.2 , the whole apartment $\mathscr{A}$ spanned by $(e)$ consists of logarithmic lattices, whereas Theorem 44 implies that $\mathscr{A}$ contains a trivializing lattice $M$. The vector bundle $\mathfrak{D}^{M}$ is therefore both logarithmic and trivial.

Note 58. Here we have a solution by modifying the Deligne bundle only at one point. Note that the lattice $M$ corresponds to a BG trivialization of $\mathfrak{D}$ (see Theorem 59 below). Also note that this result also holds replacing $\mathfrak{D}$ with any other weak solution to Riemann-Hilbert.

5.2.2. Trivializations of weak solutions. Let $\mathscr{E}$ be a weak solution of the RiemannHilbert problem, and let $\mathscr{F}$ be a trivialization of $\mathscr{E}$ at $x \notin \mathscr{Y}$. In a global basis of sections $(e)$ of the bundle $\mathscr{F}$, the connection $\nabla$ is expressed by the matrix of global meromorphic 1-forms $\Omega$, which has a simple pole at every $s \in \mathscr{Y}$, and an $a$ priori uncontrolled pole at $x$. Assuming for simplicity that $x \notin \mathscr{Y}$ is the point at infinity $\infty \in \mathbb{P}^{1}(\mathbb{C})$, there exist matrices $A_{i} \in \mathrm{M}_{n}(\mathbb{C})$ for $1 \leqslant i \leqslant p$ and a matrix

$$
B(z)=B_{0}+\cdots+B_{t} z^{t}
$$


such that the connection has the matrix

$$
\Omega=\left(\sum_{i=1}^{p} \frac{A_{i}}{z-s_{i}}+B(z)\right) d z .
$$

The most surprising consequence of the permutation lemma, as we state it, concerns the analytic invariants of the weak solutions to the Riemann-Hilbert problem.

Theorem 59. Let $\mathscr{E}$ be a weak solution to the Riemann-Hilbert problem for $\chi$. Then, for any $x \notin \mathscr{Y}$, there exists a $B G$ trivialization $\mathscr{F}$ of $\mathscr{E}$ at $x$ which is also logarithmic at $x$. Let $Y=\Gamma(X, \mathscr{F})$ and let $\psi_{s}=\operatorname{Res}_{s}^{\mathscr{F}} \nabla \in \operatorname{End}(Y)$.

(1) The map $\Psi=\sum_{s \in \mathscr{S}} \psi_{s}=-\operatorname{Res}_{x} \nabla$ is semisimple, and has integer eigenvalues, which are equal to the type of the bundle $\mathscr{E}$.

(2) The image of the Harder-Narasimhan filtration of $\mathscr{E}$ in $Y$ is equal to the flag induced by the eigenspaces of $\Psi$ ordered by increasing values.

Proof. If $x \notin \mathscr{Y}$, the monodromy at $x$ is trivial, and the stalk $\mathscr{E}_{x}$ of $\mathscr{E}$ coincides with $\mathfrak{D}_{x}$. The Birkhoff form $\Upsilon$ of $D$ (which is then unique) is equal to the space $V^{\nabla}$ of horizontal sections at $x$. All flags in $\mathbf{D}=\mathfrak{D}_{x} / \mathfrak{m}_{x} \mathfrak{D}_{x}$ are stable under $\operatorname{Res}_{x}^{\mathfrak{D}} \nabla=0$. According to Corollary 46, the $\Upsilon$-lifting of the flag induced by any BG trivialization of $\mathscr{E}$ at $x$ is a logarithmic $B G$ trivialization of $\mathscr{E}$ at $x$. In a global basis of sections (e) of $\mathscr{F}$, the connection has the matrix

$$
\begin{aligned}
A & =\sum_{s \in \mathscr{S} \backslash\{\infty\}} \frac{A_{s}}{z-s}+\frac{B}{z-x} \quad\left(\text { where } B=-\sum_{s \in \mathscr{S}} A_{s} \text { if } x \neq \infty\right) \\
& =\sum_{s \in \mathscr{S}} \frac{A_{s}}{z-s} \quad \text { if } x=\infty \notin \mathscr{Y},
\end{aligned}
$$

since $\nabla$ has no other singularities outside $\mathscr{S} \cup\{x\}$. The eigenvalues of $-B=$ $\sum_{s \in \mathscr{S}} A_{s}$ are therefore equal to the type of $\mathscr{E}$, and the Harder-Narasimhan filtration is defined by the blocks of equal eigenvalues ordered by increasing values.

As a consequence, we deduce the following new sufficient condition for the solubility of the strong Riemann-Hilbert problem.

Corollary 60. Let $\mathscr{E} \in \mathscr{H}$ and let $\mathfrak{D}$ be the Deligne lattice of $(\mathscr{V}, \nabla)$. Let $x \in X$, such that $\mathscr{E}_{x}=\mathfrak{D}_{x}=\Delta$. Let $\mathbf{D}=\Delta / \mathfrak{m}_{x} \Delta$. Let $\mathscr{F}_{F} \in \mathrm{BG}_{x}(\mathscr{E})$, and $M=\mathscr{F}_{x}$. If the flag $\boldsymbol{F}^{\Delta}(M)$ induced in $D$ is stable under $\operatorname{Res}_{x}^{\mathfrak{D}} \nabla \in \operatorname{End}(\mathbf{D})$, then there exists $\widetilde{\mathscr{F}} \in \mathrm{BG}_{x}(\mathscr{E})$ which is moreover logarithmic at $x$.

Proof. Let $\tilde{M}$ be the $\Upsilon$-lifting of the flag $\boldsymbol{F}^{\Delta}(M)$, where $\Upsilon$ is a Birkhoff form of $\Delta$. According to Proposition 52, the lattice $\tilde{M}$ is logarithmic, and by the permutation lemma, it is a BG trivializing lattice. Therefore, the bundle $\mathscr{E} \tilde{M}$ satisfies the conclusions of the corollary. 
At this point, we would like to sum up our findings about trivial bundles in the following proposition.

Proposition 61. Let $\mathscr{F} \in \mathscr{H}_{0}$ be a trivial bundle in $\mathscr{V}$, and let $Y=\mathscr{F}(X)$ be the $\mathbb{C}$-vector space of global sections. Let $x \in X$, and $\mathscr{E} \in H$ such that $\mathscr{F} \sim_{x} \mathscr{E}$. Let moreover $\Lambda=\mathscr{E}_{x}$ and $M=\mathscr{F}_{x}$.

(i) $Y$ has a well-defined flag $\mathrm{HN}_{\mathscr{E}}$ induced by the Harder-Narasimhan filtration of $\mathscr{E}$.

(ii) If $\mathscr{F} \in \mathrm{BG}_{x}(\mathscr{E})$, then $T(\mathscr{E}) \cong \mathbf{E D}_{\Lambda}(M)$ and any Smith basis of $Y$ for $\Lambda$ ordered according to $K^{\searrow}$ is strictly adapted to $\mathrm{HN}_{\mathscr{E}}$.

(iii) If $\mathscr{F}$ is additionally logarithmic at $x$, and the stalk $\mathscr{E}_{x}$ coincides with the Deligne lattice $\mathfrak{D}_{x}$, then the type $T(\mathscr{E})$ is given by the integer parts of the eigenvalues of the residue $\operatorname{Res}_{x}^{\mathscr{F}} \nabla \in \operatorname{End}(Y)$, that is, of the exponents of $\nabla$ on F at $x$.

(iv) Finally, if $\mathscr{E} \in \mathrm{RH}_{\chi}$ is moreover a weak solution to Riemann-Hilbert, then

$$
\sum_{x \in X} \operatorname{Res}_{x}^{\mathscr{F}} \nabla=0 .
$$

When $(\mathscr{E}, \mathscr{F})$ satisfy (i) to (iv), we say that $\mathscr{F}$ is a good RH trivialization of $\mathscr{E}$ at $x$.

Let $\mathscr{F}$ be a good RH trivialization of $\mathscr{E}$ at $x \notin \mathscr{Y}$. Let $(\sigma)$ be any basis of $Y=$ $\Gamma(X, \mathscr{F})$. In $(\sigma)$, the connection has a matrix of the form (21). The identification of $Y$ to $\mathbb{C}^{n}$ by means of $(\sigma)$ endows $\mathbb{C}^{n}$ with $p+1$ linear maps $\psi_{s}$ for $s \in \mathscr{S}^{*}=$ $\mathscr{S} \cup\{x\}$, that we can identify with the matrices $\widetilde{L}_{s}$ for $s \in \mathscr{S}$ and $-\sum_{s \in \mathscr{S}} \widetilde{L}_{s}$ for $s=x$. With these notations, we set the following definition.

Definition 62. The space $\mathbb{C}^{n}$, endowed with the maps $\psi_{s}$ for $s \in \mathscr{S}^{*}$, is called a linear Fuchsian model of $\mathscr{E}$.

With this notion, we can reduce some questions about vector bundles to linear algebra statements. For instance we can give the following computable version of a criterion due to Gabber for the reducibility of the triviality index originally appearing in [Sabbah 2002, Corollary I.4.14], that we state here only for the case of a logarithmic modification.

Corollary 63. Let $\mathscr{E} \in \mathrm{RH}_{\chi}$ be a weak solution, and consider a linear Fuchsian model at $x \notin \mathscr{Y}$, given by $p$ matrices $A_{s}$ for $s \in \mathscr{Y}$ such that

$$
\sum_{s \in \mathscr{S}} A_{s}=\operatorname{diag}\left(\boldsymbol{t}_{1} I_{n_{1}}, \ldots, \boldsymbol{t}_{s} I_{n_{s}}\right)
$$

where the integers $\boldsymbol{t}_{\boldsymbol{i}}$ satisfy $\boldsymbol{t}_{\boldsymbol{i}}>\boldsymbol{t}_{\boldsymbol{i}+1}$, in such a way that the flag $\mathrm{HN}$ is the flag $0=F_{0} \subset F_{1} \subset \cdots \subset F_{s}=\mathbb{C}^{n}$ having signature $\left(n_{1}, \ldots, n_{s}\right)$ in the canonical basis of $\mathbb{C}^{n}$. There exists a weak solution $\mathscr{E}^{\prime}$ adjacent to $\mathscr{E}$ at $s \in X$ and such that 
$i\left(\mathscr{E}^{\prime}\right)<i(\mathscr{E})$ if and only if there exists an $A_{s}$-stable subspace $W \subset \mathbb{C}^{n}$ such that $W \cap F_{1}=(0)$.

Proof. Let $T=\operatorname{diag}\left(\boldsymbol{t}_{1} I_{n_{1}}, \ldots, \boldsymbol{t}_{s} I_{n_{s}}\right)=\operatorname{diag}\left(t_{1}, \ldots, t_{n}\right)$ be the type of $\mathscr{E}$. We have $i(\mathscr{E})=\sum_{i=1}^{s} n_{i}\left(\boldsymbol{t}_{1}-\boldsymbol{t}_{i}\right)$. According to Proposition 28, any adjacent weak solution $\mathscr{E}^{\prime}$ is given by an $A_{s}$-stable subspace $W \subset \mathbb{C}^{n}$. For any basis $(e)$ of $\mathbb{C}^{n}$ respecting the flag $\mathrm{HN}$, the bundle $\mathscr{E}^{\prime}$ has type $T^{\prime}=T-K$, where $k_{i}=0$ when $e_{i} \in W$ and $k_{i}=1$ otherwise; therefore $i\left(\mathscr{E}^{\prime}\right)=\sum_{i=1}^{n}\left(\max \left(t_{i}-k_{i}\right)-t_{i}+k_{i}\right)$ where $t_{i}$ represent the elements of $T$ without multiplicities. Accordingly, we have

$$
i(\mathscr{E})-i\left(\mathscr{\mathscr { C }}^{\prime}\right)=\sum_{i=1}^{n}\left(t_{1}-k_{i}-\max \left(t_{i}-k_{i}\right)\right) .
$$

Now, if there exists $i$ such that $t_{i}=\boldsymbol{t}_{1}$ and $k_{i}=0$, then $\max \left(t_{i}-k_{i}\right)=\boldsymbol{t}_{1}$; thus $i(\mathscr{E})-i\left(\mathscr{E}^{\prime}\right)=\sum_{i=1}^{n}-k_{i}<0$ (because we exclude the trivial case $W=\mathbb{C}^{n}$ ). Otherwise we have $\max \left(t_{i}-k_{i}\right)=\boldsymbol{t}_{1}-1$, and then $i(\mathscr{E})-i\left(\mathscr{E}^{\prime}\right)=\sum_{i=1}^{n}\left(1-k_{i}\right)>0$. Therefore $\mathscr{C}^{\prime}$ exists if and only if there exists $W$ stable under some $A_{s}$ such that $W \cap F_{1}=0$.

Proposition 64. Let $\mathscr{F}$ be a $B G$ trivialization of $\mathfrak{D}$ at $x \notin \mathscr{Y}$. If there exists a flag $\boldsymbol{F}$ in $Y=\mathscr{F}(X)$ which is transversal to $\mathrm{HN}_{\mathfrak{D}}$, and is moreover stable under the action of one of the maps $\psi_{s}$ for $s \in \mathscr{Y}$, then the strong Riemann-Hilbert problem has a solution, which moreover coincides with $\mathfrak{D}$ outside $s$.

Proof. Let $\boldsymbol{F}$ be a flag of $Y$, which is stable under $\psi_{s}$. Taking stalks at $x$ of a $\mathbb{C}$-basis of $F$, we can see the flag $\boldsymbol{F}$ in $\mathbf{D}=\mathfrak{D}_{s} / \mathfrak{m}_{s} \mathfrak{D}_{s}$. According to Lemma 27(ii), there exists a BG trivialization $\mathscr{E}$ of $\mathfrak{D}$ at $x$, whose image in $D=\mathfrak{D}_{s} / \mathfrak{m}_{s} \mathfrak{D}_{s}$ is $\boldsymbol{F}$. Let $(e)$ be a BG basis of $\mathfrak{D}_{s}$ with respect to $\mathscr{E}_{s}$. Consequently, its image in D respects the flag $\boldsymbol{F}$. Let $\Upsilon$ be a Birkhoff form of $\mathfrak{D}_{s}$, and let $\left(e_{\Upsilon}\right)$ be the $\Upsilon$-basis of (e). Since the gauge from $(e)$ to $(e \Upsilon)$ is tangent to $I$, the lattice $M$ induced from $\left(e_{\Upsilon}\right)$ by the elementary divisors $K$ of $\mathscr{E}_{S}$ in $\Lambda$ is also a trivializing BG lattice for $\mathfrak{D}$ at $s$. However, the lattice $M$ is also logarithmic, since by construction it induces in $\mathbf{D}$ the $\psi_{s}$-stable flag $\boldsymbol{F}$, and moreover sits inside an apartment that contains the Birkhoff form $\Upsilon$. Hence, the bundle $\mathfrak{D}^{M}$ is both trivial and logarithmic.

We have represented the weak solutions to the Riemann-Hilbert problem as points in a product of subvarieties of stable flags.

Theorem 65. Let $\mathfrak{D}$ be the Deligne bundle, and $\mathscr{F}$ a BG trivialization at an apparent singularity $x \notin \mathscr{Y}$. The set of weak solutions to the Riemann-Hilbert problem for $\chi$ is parametrized by the set

$$
\mathrm{RH}_{\chi}=\left\{\left(\boldsymbol{F}^{s}, D_{s}\right)_{s \in \varphi} \mid \boldsymbol{F}^{s} \in \mathfrak{F l}_{\psi_{s}}(Y), D_{s} \in \mathbb{Z}^{n}\left(\boldsymbol{F}^{s}\right)\right\},
$$

where $Y=\mathscr{F}(X)$ and $\psi_{s}=\operatorname{Res}_{s}^{\mathscr{F}} \nabla \in \operatorname{End}_{\mathbb{C}}(Y)$ for $s \in \mathscr{Y}$. 
5.3. The type of the Deligne bundle. The strong version of the Riemann-Hilbert problem would directly have a solution if the Deligne bundle were trivial. However, this is not the case, unless all singular points are apparent, since the exponents of $\nabla$ are normalized in such a way that their sum is nonnegative. This means that the type of the Deligne bundle as a rule is not trivial. We have seen several ways to characterize this nontriviality. The type characterizes the isomorphism classes of holomorphic vector bundles, so it would seem possible to work with this sole information. However, we are not in the right category to do so, since we consider holomorphic bundles with an embedding in a meromorphic one, denoted by $\mathscr{V}$. This is the reason for which there are several trivial bundles in $\mathscr{V}$. From another point of view, it is not possible to determine on the sole basis of the sequence $T=\left(a_{1}, \ldots, a_{n}\right)$, what the effect of changing the stalk of $\mathfrak{D}$ at $x$ will be. Obviously the geometry of the Harder-Narasimhan filtration will play a decisive role.

5.3.1. Trivializations of the Deligne bundle. Let us examine in further detail the case of the Deligne bundle $\mathfrak{D}$. Let us say that $\delta_{i}$ is an elementary generator of the homotopy group $G=\pi_{1}\left(X \backslash \mathscr{Y}, z_{0}\right)$, if $\delta_{i}$ is a closed path based at $z_{0}$, having winding number +1 around the singularity $s_{i}$ and 0 around the others. Let $G_{i}=\chi\left(\delta_{i}\right)$ and $L_{i}=\frac{1}{2 i \pi} \log G_{i}$, normalized as for the Deligne lattice. Let $\left(\sigma_{i}\right)$ be a basis of the Birkhoff form $\Upsilon_{i}$ at $s_{i}$ described in Note 55, such that the connection has locally as matrix $\Omega_{i}=L_{i} \frac{d z}{z}$, on a neighborhood, say $D_{i}$ of $s_{i}$. On the other hand, let $D_{0}$ be a neighborhood of $z_{0}$, and consider a basis $\left(\sigma_{0}\right)$ of the local Birkhoff form. According to what precedes, $\left(\sigma_{0}\right)$ is a basis of local $\nabla$-horizontal sections of $\mathfrak{D}$ over $D_{0}$. One can moreover choose this basis in such a way that the monodromy of $\left(\sigma_{0}\right)$ around $s_{i}$ is exactly given by the matrix $G_{i}$.

Assume now for simplicity that $x \notin \mathscr{Y}$ is the point at infinity $\infty \in \mathbb{P}^{1}(\mathbb{C})$, and let $\mathscr{F}$ be a trivialization of $\mathfrak{D}$ at $x$. In a global basis of sections $(e)$ of the bundle $\mathscr{F}$, there exist matrices $B_{i} \in \mathrm{M}_{n}(\mathbb{C})$ and a matrix

$$
B(z)=B_{0}+\cdots+B_{t} z^{t} \text { and } \quad C_{i} \in \mathrm{GL}_{n}(\mathbb{C}) \text { for } 1 \leqslant i \leqslant p
$$

such that the connection has the matrix

$$
\Omega=\left(\sum_{i=1}^{p} \frac{C_{i}^{-1} L_{i} C_{i}}{z-s_{i}}+B(z)\right) d z .
$$

Note 66. If the bundle $\mathscr{F}$ is moreover logarithmic at $\infty$ - which can be achieved, for example, by Plemelj's theorem - then $B=0$ and the residue at infinity, $L_{\infty}=$ $-\sum_{i=1}^{p} C_{i}^{-1} L_{i} C_{i}$, is semisimple with integer eigenvalues (ssie). At the cost of a (harmless) global conjugation, we can already assume that

$$
L_{\infty}=\operatorname{diag}\left(b_{1} I_{n_{1}}, \ldots, b_{s} I_{n_{s}}\right) \quad \text { with } b_{1}<\cdots<b_{s} .
$$


Note that the sequence $\mathscr{B}=\left(b_{1} I_{n_{1}}, \ldots, b_{s} I_{n_{s}}\right)$ coincides with the elementary divisors of the stalk $\mathscr{F}_{\infty}$ in $\mathfrak{D}_{\infty}$.

Definition 67. We say that $\left(C_{1}, \ldots, C_{p}\right) \in \mathrm{GL}_{n}(\mathbb{C})^{p}$ is a normalizing $p$-tuple for $\chi$ if $\sum_{i=1}^{p} C_{i}^{-1} L_{i} C_{i}$ is ssie for some (and therefore any) normalized logarithms $L_{i}$ of the generators $\chi\left(\gamma_{i}\right)$ of the monodromy group.

Normalizing $p$-tuples always exist. Putting $t$ as the coordinate $1 / z$ at infinity, the Taylor expansion of $\nabla$ at $x=\infty$ has then the nice expression

$$
\Omega=-\sum_{k \geqslant 0} \sum_{i=1}^{p} s_{i}^{k} \widetilde{L}_{i} t^{k} \frac{d t}{t} \quad \text { with } \widetilde{L}_{i}=C_{i}^{-1} L_{i} C_{i} .
$$

We have thus reduced the computation of the type of the Deligne bundle to the computation of the matrices $C_{i}$ (the so-called connection matrices, because they connect the different local expressions of $\nabla$ on the local Birkhoff forms). It is however well known that the computation of the connection matrices is difficult. Any other trivialization of $\mathfrak{D}$ at infinity is given by a monopole gauge (as coined in [Ilyashenko and Yakovenko 2007]), namely a unimodular polynomial matrix $\Pi \in \mathrm{GL}_{n}(\mathbb{C}[z])$, that is, a matrix satisfying

$$
\Pi=P_{0}+P_{1} z+\cdots+P_{k} z^{k} \quad \text { such that } \operatorname{det} \Pi(z)=\mathrm{cst} \in \mathbb{C}^{*} .
$$

Proposition 68. Given a family of points $s_{1}, \ldots, s_{p} \in \mathbb{C}$ and invertible matrices $C_{1}, \ldots, C_{p} \in \mathrm{GL}_{n}(\mathbb{C})$, there exists a monopole gauge $\Pi \in \mathrm{GL}_{n}(\mathbb{C}[z])$ such that $\Pi\left(s_{i}\right)=C_{i}$ for $1 \leqslant i \leqslant p$.

Proof. The group $\mathrm{GL}_{n}(R)$ on a ring is generated by transformations $T_{i j}(\lambda)=$ $I+\lambda E_{i j}$ where $\lambda \in R$ and $E_{i j}$ is the $(i, j)$ element of the canonical basis of the vector space $\mathfrak{g l}_{n}$. At the cost of introducing the trivial transformations $T_{i j}(0)=I$, one can assume that all the matrices $C_{i}$ can be expressed as a product of the same transformations with different parameters:

$$
C_{i}=T_{1}\left(\mu_{1}^{i}\right) \cdots T_{s}\left(\mu_{s}^{i}\right) \quad \text { with } \mu_{t}^{i} \in \mathbb{C} .
$$

Define then $\lambda_{k} \in \mathbb{C}[z]$ such that $\lambda_{k}\left(s_{i}\right)=\mu_{k}^{i}$ for $1 \leqslant i \leqslant p$. By construction, the product $\widetilde{\Pi}=T_{1}\left(\lambda_{1}\right) \cdots T_{s}\left(\lambda_{s}\right) \in \mathrm{SL}_{n}(\mathbb{C}[z])$ indeed interpolates the matrices $C_{i}$ at the points $s_{i}$.

As a consequence of this result, one can find a trivialization $\mathscr{E}$ at infinity of the Deligne bundle such that the residues of the connection $\nabla$ are expressed in a basis of $Y=\Gamma(X, \mathscr{E})$ as the actual matrices $L_{i}$ (and not conjugated to them). Although the point at infinity of $\mathscr{E}$ is still an apparent singularity, we have no control on the Poincaré rank of $\nabla$ at $\infty$. 
The results of this section also hold (with the adequate modifications) if the apparent singularity is assumed to be located at $z_{0} \notin \mathscr{Y} \cup\{\infty\}$. We will refer to the trivialization $\mathscr{E}$ as an adapted trivialization of $\mathfrak{D}$ at $z_{0}$.

5.3.2. A Deligne-Simpson-type problem. We know that there exists a family of invertible matrices $\left(C_{i}\right)$ such that $\sum_{i=1}^{p} C_{i}^{-1} L_{i} C_{i}$ is semisimple with integer eigenvalues and that these eigenvalues are equal to the type of the Deligne bundle. This raises two questions:

(1) Does there exist a logarithmic trivialization of $\mathfrak{D}$ for any such family $\left(C_{i}\right)$ ?

(2) If there exist several families with this property, how do we recognize those that indeed give the type of the Deligne bundle?

This also raises an interesting computational problem akin to the well-known Deligne-Simpson problem (see, e.g., [Crawley-Boevey 2003]). Let $\mathscr{C}_{i}$ be the conjugacy class of $L_{i}=\frac{1}{2 i \pi} \log G_{i}$ under $\mathrm{GL}_{n}(\mathbb{C})$.

(DS) Determine all conjugates $\widetilde{L}_{i} \in \mathscr{C}_{i}$ such that $\sum_{i=1}^{p} \widetilde{L}_{i}=\operatorname{diag}\left(b_{1}, \ldots, b_{n}\right) \in \mathbb{Z}^{n}$.

5.4. The Bolibrukh-Kostov theorem. The most celebrated recent result on the Riemann-Hilbert problem is the following fact, proved first independently by A. Bolibrukh and V. Kostov.

Theorem 69 (Bolibrukh-Kostov). The strong Riemann-Hilbert problem is solvable for any irreducible monodromy representation $\chi$.

We give first an algebraic proof of a classical result of Bolibrukh [Anosov and Bolibrukh 1994, Proposition 4.2.1].

Proposition 70. If the representation $\chi$ is irreducible, then for any weak solution $\mathscr{E} \in \mathrm{RH}_{\chi}$, the type $T(\mathscr{E})=\left(t_{1}, \ldots, t_{n}\right)$ of $\mathscr{E}$ satisfies $\left|t_{i}-t_{j}\right| \leq|i-j|(p-2)$.

Proof. Assume here for simplicity that $x=\infty \notin \mathscr{Y}$, and consider again the setting of Section 5.3.1. Let $\mathscr{E}$ be any weak solution to Riemann-Hilbert, and $\mathscr{F}$ be a logarithmic BG trivialization of $\mathscr{E}$ at $x$. Let $T=\left(t_{1}, \ldots, t_{n}\right)$ be the type of $\mathscr{E}$. In a basis (e) of global sections of $\mathscr{F}$, there exist constant matrices $\widetilde{L}_{a}$ for $a \in \mathscr{Y}$ such that the connection $\nabla$ has in $(e)$ the matrix

$$
\Omega=\sum_{a \in \mathscr{S}} \frac{\tilde{L}_{a}}{z-a} d z=-\frac{d \tilde{z}}{\tilde{z}} \sum_{k \geqslant 0} \Omega_{k} \tilde{z}^{k} \quad \text { with } \Omega_{k}=\sum_{a \in \mathscr{S}} a^{k} \tilde{L}_{a} \text { and } \tilde{z}=\frac{1}{z} .
$$

By Proposition 49, the shearing $\tilde{z}^{-T}$ suppresses the singularity at $x$, since the basis $\tilde{z}^{-T}(e)$ spans the Deligne lattice. As a consequence, $\widetilde{\Omega}=\tilde{z}^{T} \Omega(\tilde{z}) \tilde{z}^{-T}+T \frac{d \tilde{z}}{\tilde{z}}$ must satisfy $v(\widetilde{\Omega}) \geq 0$. Therefore, the residue matrix $B=-\sum_{a \in \mathscr{S}} \widetilde{L}_{a}$ of $\Omega$ at $x$ is diagonal and equal to $-T$. We can assume further that

$$
B=\operatorname{diag}\left(b_{1} I_{n_{1}}, \ldots, b_{s} I_{n_{s}}\right) \quad \text { with } b_{1}=-\boldsymbol{t}_{1}<\cdots<b_{s}=-\boldsymbol{t}_{s}
$$


where $\left(\boldsymbol{t}_{1} I_{n_{1}}, \ldots, \boldsymbol{t}_{s} I_{n_{s}}\right)$ represents the type of $\mathscr{E}$ with multiplicities. Partition any matrix $M$ according to the eigenvalue multiplicities of $B$, as $\left(M_{\ell, m}\right)$ for $1 \leq \ell, m \leq s$. Then the matrix of the connection can be rewritten by blocks as

$$
\tilde{\Omega}_{\ell, m}=\Omega_{\ell, m} \boldsymbol{t}^{\boldsymbol{t}_{\ell}-\boldsymbol{t}_{m}}+T \frac{d \tilde{z}}{\tilde{z}}=\left(-\sum_{j \geq 0} \Omega_{\ell, m}^{(j)} \tilde{z}^{j+\boldsymbol{t}_{\ell}-\boldsymbol{t}_{m}}+\delta_{\ell, m} \boldsymbol{t}_{\ell} I_{n_{\ell}}\right) \frac{d \tilde{z}}{\tilde{z}} .
$$

For each $(\ell, m)$ block, this series must have strictly positive valuation. The sum $\sum_{a \in \mathcal{S}} \tilde{L}_{a}=T$ imposes conditions on all blocks of the residues $\widetilde{L}_{a}$, while when $\ell>m$ we get the following equations:

$$
\Omega_{\ell, m}^{(j)}=\sum_{a \in \mathscr{S}} a^{j}\left(\tilde{L}_{a}\right)_{\ell, m}=0 \text { for } 0 \leq j \leq \boldsymbol{t}_{m}-\boldsymbol{t}_{\ell} \quad \text { when } \ell>m .
$$

For a fixed pair $(\ell, m)$, let $k=\max \left(0, \boldsymbol{t}_{m}-\boldsymbol{t}_{\ell}\right)$, and let $X_{i} \in \mathbb{C}^{n_{\ell} \times n_{m}}$ be the $(\ell, m)$-block of the matrix $\widetilde{L}_{s_{i}}$, for $1 \leq i \leq p$. For $1 \leq \alpha \leq n_{\ell}$ and $1 \leq \beta \leq n_{m}$, let $v_{\alpha, \beta} \in \mathbb{C}^{p}$ be the vector constructed by taking the coefficient of index $(\alpha, \beta)$ of $X_{i}$, for $1 \leq i \leq p$. Then, the equations (22) can be reformulated as

$$
v_{\alpha, \beta} \in \operatorname{ker} M_{k}(\underline{s}) \quad \text { where } M_{k}(\underline{s})=\left(\begin{array}{ccc}
1 & \cdots & 1 \\
s_{1} & \cdots & s_{p} \\
\vdots & & \vdots \\
s_{1}^{k} & \cdots & s_{p}^{k}
\end{array}\right) .
$$

The matrix $M_{k}(\underline{s})$ is an upper-left submatrix of a Vandermonde matrix with coefficients

$$
\underline{s}=\left(s_{1}, \ldots, s_{p}\right) \in \mathbb{C}^{p} \backslash \bigcup_{i \neq j}\left\{x_{i} \neq x_{j}\right\} .
$$

Since all the $s_{i}$ are distinct, this matrix has always full rank. In particular, as soon as $\boldsymbol{t}_{m}-\boldsymbol{t}_{\ell} \geq p-1$, it has a null kernel, and so all the blocks $X_{i}$ are zero. Due to the ordering of the $\boldsymbol{t}_{i}$, we also have $\boldsymbol{t}_{m^{\prime}}-\boldsymbol{t}_{\ell^{\prime}} \geqslant p-1$ for $m^{\prime} \leqslant m$ and $\ell^{\prime} \geqslant \ell$; thus all matrices $\widetilde{L}_{a}$ have a lower-left common zero block. If $m=\ell+1$, this means that the representation $\chi$ is reducible.

Proof of Theorem 69. Consider the Deligne bundle $\mathfrak{D}$ and an arbitrary singularity $s \in \mathscr{Y}$. Put $\Delta=\mathfrak{D}_{s}$ and let $\boldsymbol{F}$ be a complete flag in $\Delta / \mathfrak{m} \Delta$ which is stable under $\delta=\operatorname{Res}_{s}^{\mathfrak{D}} \nabla$. Let $D=(0, d, 2 d, \ldots,(n-1) d)$. By Proposition 52, the lattice $\Lambda$ obtained by lifting the pair $(\boldsymbol{F}, D)$ in the $z$-Birkhoff form $\Upsilon \subset \Delta$ is logarithmic. Let $(e)$ be a basis of $\Upsilon$ respecting the flag $\boldsymbol{F}$. Since the residue $\delta$ is upper-triangular in (e), and the elements of $D$ are distinct, the elements of the matrix $\Omega=\operatorname{Mat}\left(\nabla,\left(z^{D} e\right)\right)$ outside the diagonal have valuation at least $d$. Hence $\Omega=\left(A+z^{d} U\right) \frac{d z}{z}$ where $A$ is diagonal and $U$ holomorphic. By Theorem 44, the 
apartment $\mathscr{A}$ spanned by (e) contains a Birkhoff-Grothendieck trivialization $M$ of $\Lambda$, as in the following figure:

$$
\Delta \stackrel{z^{D}}{\longrightarrow} \Lambda \in \Lambda_{\log } \stackrel{z^{\widetilde{D}}}{\longrightarrow} M \in \operatorname{BG}(\Lambda) .
$$

According to Proposition 70, the sequence $\widetilde{D}$ satisfies $\Delta \widetilde{D} \leqslant(n-1)(p-2)$, while the Poincaré rank $\mathfrak{p}$ of $\nabla$ on $M$ satisfies $\mathfrak{p}=\min \left(0,-v\left(z^{-\widetilde{D}} \Omega z^{\widetilde{D}}\right)\right)$. Since $v\left(z^{-\widetilde{D}} \Omega z^{\widetilde{D}}\right) \geqslant d-\Delta \widetilde{D}$ holds, it is sufficient to impose $d \geqslant(n-1)(p-2)$ to ensure that $\mathfrak{p}=0$, that is, that the lattice $M$ is logarithmic. Then $\mathfrak{D}^{M}$ is a strong solution.

The previous proof holds in fact for any representation $\chi$ for which there exists a constant $R$ such that for any weak solution $\mathscr{E}$ of RH for $\chi$, the type of $\mathscr{E}$ satisfies $\Delta T(\mathscr{E}) \leqslant R$. Finally, as a byproduct of the proof of Proposition 70, we have the following result.

Corollary 71. Let $\tilde{L}_{i} \in \mathscr{C}_{i}$ such that $\sum_{i=1}^{p} \tilde{L}_{i}=\operatorname{diag}\left(t_{1}, \ldots, t_{n}\right) \in \mathbb{Z}^{n}$. Then the sequence $T=\left(t_{1}, \ldots, t_{n}\right)$ represents the type of the Deligne bundle of the monodromy representation $\chi$ of the Fuchsian system (with singular locus $\mathscr{Y}=$ $\left.\left\{s_{1}, \ldots, s_{p}\right\} \subset \mathbb{C}\right)$

$$
Y^{\prime}=\sum_{i=1}^{p} \frac{\tilde{L}_{i}}{z-s_{i}} Y
$$

if and only if $\sum_{i=1}^{p} s_{i}^{j}\left(\tilde{L}_{i}\right)_{\ell, m}=0$ holds for $0 \leq j \leq k_{m}-k_{\ell}$ and $\ell>m$.

This gives a partial answer to our question (2) from Section 5.3.2. For any explicit solution $L=\left(L_{1}, \ldots, L_{p}\right)$ to the generalized Deligne-Simpson problem, we can generate nontrivial explicit examples of Deligne bundles (and their HarderNarasimhan filtrations) corresponding to monodromy representations which are locally conjugate to the original one. In the following section, we give the algorithmic procedures that can then be used to determine effectively if the corresponding representation admits or not a strong solution to the Riemann-Hilbert problem. Note that the equations in Corollary 71 show that the set of singular loci $\mathscr{Y}$ for which $L$ corresponds to a logarithmic BG trivialization of a Deligne bundle is an algebraic (projective) subvariety of $\mathbb{C}^{p} \backslash \bigcup_{i \neq j}\left\{x_{i}=x_{j}\right\}$.

5.5. Testing the solubility of the Riemann-Hilbert problem. In this section, we apply the results of this paper to the experimental investigation of the solubility of the Riemann-Hilbert problem. We present two ways to search the space of weak solutions, which are completely effective (up to the known problem of connection matrices): one that follows paths of adjacent logarithmic lattices, based on Lemma 51, the other that uses the characterization as stable flags given in 
Proposition 52. Note that, if any (not necessarily logarithmic) trivial holomorphic bundle of the meromorphic solution to Riemann-Hilbert is explicitly given, the procedures that we present, coupled with classical Poincaré rank reduction methods, implemented on a computer algebra system, allow to make the actual computations. We however do not know if this bypasses the problem of the connection matrices.

Let $\mathfrak{D}$ be the Deligne bundle of the representation $\chi$. Let $x \notin \mathscr{Y}$, and consider a logarithmic BG trivialization $\mathscr{F}$ of $\mathfrak{D}$ at $x$. Let $Y=\Gamma(X, \mathscr{F})$ and choose a basis $(\sigma)$ of $Y$ in which the residue matrix at $x$ is equal to the diagonal that represents the type of $\mathfrak{D}$ :

$$
\operatorname{Mat}\left(\operatorname{Res}_{x}^{\mathscr{F}} \nabla,(\sigma)\right)=-D=\operatorname{diag}\left(-k_{1} I_{n_{1}}, \ldots,-k_{s} I_{n_{s}}\right) \quad \text { where } k_{1}>\cdots>k_{s} .
$$

In the basis $(\sigma)$, the connection has a matrix of the form (21), and the HarderNarasimhan filtration is expressed as the flag $\mathrm{HN}_{Y}$ of signature $\left(n_{1}, \ldots, n_{s}\right)$ of $Y$. Let $V=\Gamma(X, \mathscr{V})$ be the $\mathscr{K}$-vector space of meromorphic sections of $\mathscr{V}$, where $\mathscr{K}=\Gamma\left(X, \mu_{X}\right)$ is the field of meromorphic functions on $X$.

For $s \in \mathscr{Y}$, let $t$ be a coordinate at $x$ with divisor $(t)=x-s$, and $(\widetilde{\sigma})=t^{-D}(\sigma)$. Recall that $t^{-1}$ is a coordinate at $s$. For clarity's sake, we will put $t_{x}=t$ and $t_{s}=t^{-1}$ when we are dealing with local sections. Let $\widetilde{\mathscr{F}}=t_{S}(\mathscr{F})$ be the transport of $\mathscr{F}$ at $s$ and $\tilde{Y}=\Gamma(X, \widetilde{\mathscr{F}})$. We regard $Y$ and $\tilde{Y}$ as sub- $\mathbb{C}$-vector spaces of $V$, spanned respectively by the $\mathscr{K}$-bases $(\sigma)$ and $(\widetilde{\sigma})$ of $V$. The relation $(\widetilde{\sigma})=t^{-D}(\sigma)$ induces a well-defined fixed isomorphism between $Y$ and $\tilde{Y}$.

Claim 72. The trivial bundle $\widetilde{\mathscr{F}}$ is a $B G$ trivialization of $\mathfrak{D}$ at $s$.

Claim 73. The flag $\mathrm{HN}_{\tilde{Y}}$ is the flag of signature $\left(n_{1}, \ldots, n_{s}\right)$ spanned by $(\widetilde{\sigma})$.

Claim 74. The germ $\left(\sigma_{s}\right)$ of the global basis of $Y$ at $s$ is a local basis of $\mathfrak{D}_{s}$.

Indeed, we have the two dual schematic representations, where $\left(\sigma_{x}\right): \mathscr{E}_{x}$ means that $(\sigma)$ is a local basis of $\mathscr{E}$ at $x$ and $(\sigma): Y$ means that $(\sigma)$ is a global basis of the form $Y$ :

$$
\left(\widetilde{\sigma}_{x}\right): \mathfrak{D}_{x} \stackrel{t_{x}^{D}}{\longrightarrow}(\sigma): Y \quad \text { and } \quad\left(\sigma_{s}\right): \mathfrak{D}_{s} \stackrel{t_{s}^{D}}{\longrightarrow}(\widetilde{\sigma}): \tilde{Y} .
$$

5.5.1. Bolibrukh's first counterexample. Bolibrukh's first published counterexample is the $3 \times 3$ system

$$
d X / d z=A X,
$$

$$
A=\left(\begin{array}{rrr}
0 & 1 & 0 \\
0 & z & 0 \\
0 & 0 & -z
\end{array}\right) \frac{1}{z^{2}}+\left(\begin{array}{rrr}
0 & 1 & 0 \\
0 & -\frac{1}{6} & \frac{1}{6} \\
0 & -\frac{1}{6} & \frac{1}{6}
\end{array}\right) \frac{1}{z+1}+\left(\begin{array}{rrr}
0 & 0 & 1 \\
0 & -\frac{1}{2} & -\frac{1}{2} \\
0 & \frac{1}{2} & \frac{1}{2}
\end{array}\right) \frac{1}{z-1}+\left(\begin{array}{rrr}
0 & -1 & -1 \\
0 & -\frac{1}{3} & \frac{1}{3} \\
0 & -\frac{1}{3} & \frac{1}{3}
\end{array}\right) \frac{1}{z-\frac{1}{2}} .
$$

Let us show what the different notions introduced in the paper are in this case. We consider the system (23) to be the expression of the connection $\nabla$ on a trivial 
bundle $\mathscr{E}$, in a basis $(\sigma)$ of global sections. The singular divisor is $\mathscr{D}=2 \cdot 0+1-1+\frac{1}{2}$ and the matrices at $1,-1$ and $\frac{1}{2}$ are nilpotent. The point at infinity is not singular, since, putting $t=1 / z$, we have

$$
-\frac{1}{t^{2}} A\left(\frac{1}{t}\right)=\frac{1}{2}\left(\begin{array}{rrr}
-1 & 0 & -1 \\
-1 & 0 & 1 \\
1 & 0 & 1
\end{array}\right)+o(1)
$$

Therefore the stalks $\mathscr{E}_{x}$ for $x \in \mathbb{P}^{1}(\mathbb{C}) \backslash\{0\}$ coincide with the Deligne bundle $\mathfrak{D}$ of $\nabla$ : in the terms of Section 1 , the bundle $\mathscr{E}$ is a trivialization of $\mathfrak{D}$ at 0 . However, the singular point 0 is not an apparent singularity. The gauge $P=\operatorname{diag}(1, z, 1 / z)$ brings the system to the form

$$
A_{[P]}=\frac{1}{z} \tilde{A}_{0}+\frac{1}{z+1} \tilde{A}_{-1}+\frac{1}{z-1} \tilde{A}_{1}+\frac{1}{z-\frac{1}{2}} \tilde{A}_{\frac{1}{2}}+B
$$

where

$$
\begin{gathered}
\tilde{A}_{0}=\left(\begin{array}{rrr}
0 & 1 & 1 \\
0 & 0 & -1 \\
0 & 0 & 0
\end{array}\right) \tilde{A}_{-1}=\frac{1}{6}\left(\begin{array}{rrr}
0 & -6 & 0 \\
0 & -1 & -1 \\
0 & -1 & 1
\end{array}\right) \tilde{A}_{1}=\frac{1}{2}\left(\begin{array}{lrr}
0 & 0 & 2 \\
0 & -1 & 1 \\
0 & -1 & 1
\end{array}\right), \\
\tilde{A}_{\frac{1}{2}}=\frac{1}{12}\left(\begin{array}{rrr}
0 & -6 & -24 \\
0 & -4 & 16 \\
0 & -1 & 4
\end{array}\right) \text { and } B=\frac{1}{2}\left(\begin{array}{lll}
0 & 0 & 0 \\
0 & 0 & 0 \\
0 & 1 & 0
\end{array}\right),
\end{gathered}
$$

The bundle $\mathscr{F}$ spanned by the global sections $\left(\sigma^{\prime}\right)=\left(\sigma_{1}, z \sigma_{2}, \sigma_{3} / z\right)$ is trivial by construction, and since all residues over $\mathscr{P}=\{0,1,-1,1 / 2\}$ are nilpotent, $\mathscr{F}$ is a trivialization of $\mathfrak{D}$ at $\infty$. Moreover, the stalk $\mathfrak{D}_{\infty}$ is spanned by $\left(\sigma_{\infty}\right)$, so the germ of basis $\left(\sigma_{\infty}^{\prime}\right)$ is a Smith basis of $\mathfrak{D}_{\infty}$; hence $\mathscr{F}$ is actually a BG trivialization of the Deligne bundle $\mathfrak{D}$ at $x=\infty$. In general, such a gauge can be found explicitly by combining a Poincaré rank reducing method at all finite singularities (e.g., Gérard-Levelt saturation [1973]) and the BG trivialization algorithm from Section 3.2.1.

Accordingly, the type of the Deligne bundle is $T=T(\mathfrak{D})=(1,0,-1)$. However, an apparent singularity of Poincaré rank 2 appears at $\infty$; hence the BG trivialization $\mathscr{F}$ is not logarithmic at $\infty$. To get one, we apply the permutation lemma (Proposition 38). We reorder the basis at $\infty$ as $(\widetilde{\sigma})=\left(\sigma_{3}, \sigma_{1}, \sigma_{2}\right)$ according to the decreasing elements of the type. Putting $\Lambda=\mathfrak{D}_{\infty}$ and $M=\mathscr{F}_{\infty}$, for any lattice gauge $Q \in \mathrm{GL}_{3}(\mathbb{C} \llbracket t \rrbracket)$, we can find $P^{\prime}, \widetilde{Q} \in \mathrm{GL}_{3}(\mathbb{C} \llbracket t \rrbracket)$ and a monopole 
$\Pi \in \mathrm{GL}_{3}(\mathbb{C}[z])$ as in the following diagram:

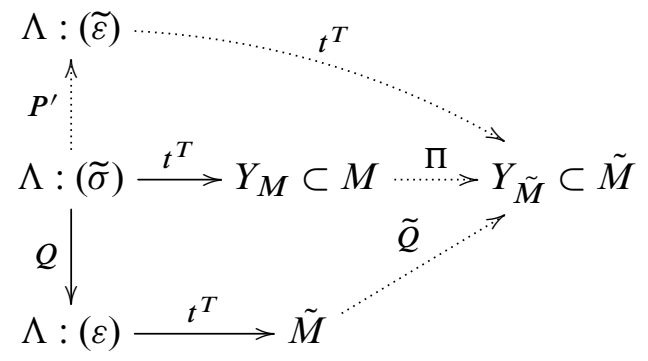

We will get a BG logarithmic trivialization if $(\varepsilon)$ is a basis of the Birkhoff form $\Upsilon$ of $\mathfrak{D}_{\infty}$. Since $\infty$ is a regular point, the gauge $Q$ is a holomorphic fundamental matrix of solutions of the system (24). Lemma 15 ensures that we can actually truncate $Q$ at order $\Delta T-1=1$; hence we can take

$$
Q=\left(\begin{array}{lll}
1 & 0 & 0 \\
0 & 1 & 0 \\
0 & 0 & 1
\end{array}\right)+\frac{1}{2}\left(\begin{array}{rrr}
-1 & 0 & -1 \\
-1 & 0 & 1 \\
1 & 0 & 1
\end{array}\right) t .
$$

The gauge $P^{\prime}$ is obtained as in the proof of Proposition 38:

$$
P^{\prime}=\left(\begin{array}{rrr}
1 & 0 & -\frac{t}{2} \\
0 & 1 & 0 \\
0 & 0 & 1
\end{array}\right) \quad \text { so that } \Pi=\left(\begin{array}{rrr}
1 & 0 & -\frac{1}{2 t} \\
0 & 1 & 0 \\
0 & 0 & 1
\end{array}\right) .
$$

Finally, we get

$$
\begin{aligned}
& A_{\left[P^{\prime} t^{T}\right]}=\frac{1}{z} A_{0}+\frac{1}{z+1} A_{-1}+\frac{1}{z-1} A_{1}+\frac{1}{z-\frac{1}{2}} A_{\frac{1}{2}}, \\
& \text { with } A_{0}=\left(\begin{array}{rrr}
0 & 0 & 0 \\
1 & 0 & 1 \\
-1 & 0 & 0
\end{array}\right), \quad A_{-1}=\frac{1}{24}\left(\begin{array}{rrr}
2 & 0 & -1 \\
0 & 0 & -24 \\
4 & 0 & -2
\end{array}\right), \\
& A_{1}=\frac{1}{8}\left(\begin{array}{rrr}
2 & 0 & 1 \\
8 & 0 & -4 \\
-4 & 0 & -2
\end{array}\right) \quad \text { and } A_{\frac{1}{2}}=\frac{1}{3}\left(\begin{array}{rrr}
2 & 0 & -1 \\
-6 & 0 & 0 \\
4 & 0 & -2
\end{array}\right) .
\end{aligned}
$$

This is a Fuchsian linear model of (23) at $x=\infty$, and we check that the eigenvalues of

$$
A_{0}+A_{-1}+A_{1}+A_{\frac{1}{2}}=\left(\begin{array}{ccc}
1 & 0 & -\frac{1}{4} \\
0 & 0 & -\frac{1}{2} \\
0 & 0 & -1
\end{array}\right)
$$


give indeed the type of $\mathfrak{D}$. The residues $A_{s}$ are all nilpotent with maximal rank; hence there is a unique complete flag $\boldsymbol{F}^{(s)}$ which is stable by $A_{s}$. In the canonical basis $(e)$ of $\mathbb{C}^{3}$, the Harder-Narasimhan filtration corresponds to the coordinate flag

$$
\boldsymbol{H}:(0) \subset\left\langle e_{1}\right\rangle \subset\left\langle e_{1}, e_{2}\right\rangle \subset \mathbb{C}^{3}
$$

and we have

$$
\begin{aligned}
\boldsymbol{F}^{(1)}=\boldsymbol{F}^{\left(\frac{1}{2}\right)} & :(0) \subset\left\langle e_{2}\right\rangle \subset\left\langle e_{2}, e_{1}+2 e_{3}\right\rangle \subset \mathbb{C}^{3}, \\
\boldsymbol{F}^{(-1)} & :(0) \subset\left\langle e_{2}\right\rangle \subset\left\langle e_{2}, e_{1}-2 e_{3}\right\rangle \subset \mathbb{C}^{3}, \\
\boldsymbol{F}^{(0)} & :(0) \subset\left\langle e_{2}\right\rangle \subset\left\langle e_{2}, e_{3}\right\rangle \subset \mathbb{C}^{3} .
\end{aligned}
$$

We see that no stable flag under any $A_{s}$ is transversal to $\boldsymbol{H}$, which is a necessary condition to be a counterexample (by Proposition 64). However, the condition of Corollary 63 is satisfied (at each $s \in \mathscr{Y}$ ), which means that there is an adjacent weak solution $\mathscr{E}$ with strictly smaller default $i(\mathscr{E})<i(\mathfrak{D})=3$.

5.5.2. Adjacent lattices. In this section, we consider a weak solution $\mathscr{E} \in \mathrm{RH}_{\chi}$. In the following proposition, we describe a procedure which allows to read off at an apparent singularity $x \notin \mathscr{Y}$, fixed once and for all, the effect on the weak solution $\mathscr{E}$ of a change of logarithmic adjacent lattice at any singularity $s \in \mathscr{T}$. More precisely, let $(\sigma)$ be a global basis of a logarithmic BG trivialization of $\mathscr{E}$ at $x$, and $\Omega$ the matrix in Fuchsian form (21) of the connection $\nabla$ in $(\sigma)$, whose residue at $x$ gives precisely the type of $\mathscr{E}$. Let $M$ be a logarithmic lattice at $s$ that is adjacent to $\mathscr{E}_{s}$. We determine explicitly a gauge transform $\Pi_{M}$ which is a monopole at $x$, such that $\Omega_{\left[\Pi_{M}\right]}$ has again Fuchsian form (21). From its semisimple residue at $x$ we read directly the type of the modified bundle $\mathscr{E}^{\mathscr{E}} M$, equal to the eigenvalues, and the Harder-Narasimhan filtration of $\mathscr{E} M$, spanned by the eigenspaces ordered by increasing values. This procedure is completely effective once the connection matrices $C_{s}$ that relate the local residue matrices $L_{s}=\frac{1}{2 i \pi} \log G_{s}$ in the Birkhoff form at $s$ and the global residue matrices $\widetilde{L}_{s}=C_{s}^{-1} L_{s} C_{s}$ in the basis $(\sigma)$ have been determined.

Let $M$ be a lattice at $s$ that is adjacent to $\mathscr{E}_{s}$. This lattice is uniquely characterized by its image $W=M / \mathfrak{m}_{s} \mathscr{E}_{s}$, that can be seen as a sub- $\mathbb{C}$-vector space $W \subset Y$. It is logarithmic if and only if $W$ is stable under the map $\operatorname{Res}_{S}^{\mathscr{E}} \nabla$.

According to Proposition 28, a BG trivialization of $\mathscr{E} M$ is obtained from a basis of $\mathscr{E}_{S}$ that simultaneously respects the space $W$ and the flag HN. Moreover, we can choose $(\varepsilon)$ in the $\mathrm{GL}_{n}(\mathbb{C})$-orbit of $(\sigma)$.

Claim 75. There exists a basis $(\varepsilon)$ of $Y$ such that $t_{s}^{D}(\varepsilon)$ spans a $B G$ trivialization of both $\mathscr{E}$ and $\mathscr{E} M$ at $s$. 
Claim 76. The matrix $P \in \mathrm{GL}_{n}(\mathbb{C})$ of the basis change from $(\sigma)$ to $(\varepsilon)$ is $(-D)$ parabolic.

Claim 77. The gauge $t_{s}^{-D} P t_{s}^{D}=t_{x}^{D} P t_{x}^{-D}$ is a monopole at $s$ and an element of $\mathrm{GL}_{n}\left(\mathrm{O}_{x}\right)$.

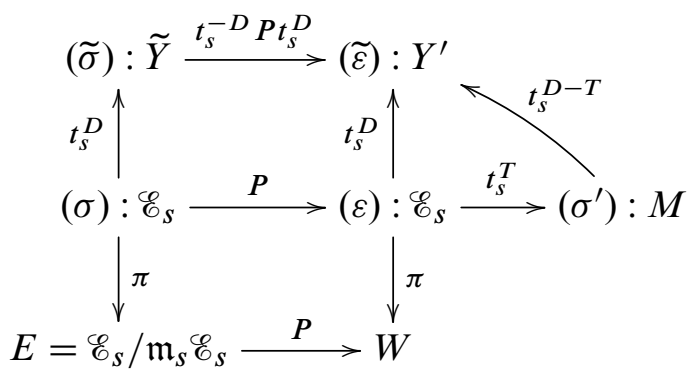

Claim 78. The basis $\left(\sigma^{\prime}\right)$ generates $M$ at $s$ and $\mathscr{E}_{y}$ at $y \neq x$.

Claim 79. The trivial bundle $\mathscr{F}^{\prime}$ spanned by $\left(\sigma^{\prime}\right)$ is a $B G$ trivialization of $\mathscr{E} M$ at $x$.

Claim 80. The gauge transform from $(\sigma)$ to $\left(\sigma^{\prime}\right)$ is $P t_{s}^{T}=P t_{x}^{-T}$.

Claim 81. The Harder-Narasimhan filtration of $\mathscr{C}^{M}$ is given by the flag of $Y^{\prime}$ spanned by $\left(\sigma^{\prime}\right)$ according to $D-T$.

Indeed, the last arrow on the right implies that at $x$, we have

$$
\left(\widetilde{\varepsilon}_{x}\right): \mathscr{E}_{x}=\mathscr{E}_{x}^{M} \stackrel{t_{x}^{D-T}}{\longrightarrow}\left(\sigma^{\prime}\right): Y^{\prime} \quad \text { where } Y^{\prime} \subset V \text { is spanned over } \mathbb{C} \text { by }\left(\sigma^{\prime}\right) .
$$

Therefore the type of $\mathscr{E}^{M}$ is, as expected, equal to $D-T$.

Proposition 82. Assume that $\mathscr{Y} \subset \mathbb{C}$ and $x=\infty$. Let $\mathscr{E} \in \mathrm{RH}_{\chi}$ be a weak solution to the Riemann-Hilbert problem. Let the connection $\nabla$ have a matrix $\Omega$ of the form (21) in a basis $(\sigma)$ of a logarithmic BG trivialization $\mathscr{F}$ of $\mathscr{E}$ at $x$. Then, for any $\widetilde{L}_{S^{-}}$stable subspace $W_{s}$ of $\mathbb{C}^{n}$, there exists a computable monopole gauge $\Pi \in \mathrm{GL}_{n}(\mathbb{C}[z])$, a constant matrix $P_{0} \in \mathrm{GL}_{n}(\mathbb{C})$ and a diagonal matrix $T$ with only 0,1 elements such that $\Omega_{\left[P_{0}(z-s)^{T} \Pi\right]}$ has again the form (21) corresponding to the modification $\mathscr{E}^{M}$, where $M$ is the lattice of $\mathscr{V}_{s}$ adjacent to $\mathscr{E}_{s}$ canonically defined by $W_{s}$.

Proof. We identify $\Gamma(X, \mathscr{F})$ with $\mathbb{C}^{n}$ by means of the basis $(\sigma)$. The residue of $\nabla$ at $s$ is then equal to the matrix $L=\widetilde{L}_{s}$ of formula (21). A logarithmic adjacent lattice $M$ is uniquely defined by an $L$-stable subspace $W \subset \mathbb{C}^{n}$. Let $(\varepsilon)$ be a basis respecting both $W$ and the Harder-Narasimhan flag $\boldsymbol{H}$, and let $P \in \mathrm{GL}_{n}(\mathbb{C})$ be the basis change from $(\sigma)$ to $(\varepsilon)$. Assume for simplicity that we have ordered the vectors $\varepsilon_{1}, \ldots, \varepsilon_{n}$ in such a way that if $\varepsilon_{i} \in H_{k} \cap W$ and $\varepsilon_{i+1} \notin W$ then $\varepsilon_{i+1} \notin H_{k}$. Let $T=\operatorname{diag}\left(t_{1}, \ldots, t_{n}\right)$ be the diagonal integer matrix defined by $t_{i}=1$ if and only if $\varepsilon_{i} \notin W$. With the simplifying assumption, the type of $\mathscr{E} M$ 
is equal to $D-T$, including the ordering condition, and the Harder-Narasimhan filtration is exactly obtained by putting together the groups of vectors corresponding to equal values of $D-T$. Therefore the basis $\left(\sigma^{\prime}\right)=(z-s)^{D-T}(\varepsilon)$ spans a BG trivialization $\mathscr{F}^{\prime}$ of $\mathscr{E}^{M}$ at $s$, and it is simultaneously a global basis of $V$. The transport $t_{x}\left(\mathscr{F}^{\prime}\right)$ is again a BG trivialization of $\mathscr{E} M$ at $x$, but it needs not be logarithmic anymore. Since $\mathscr{E}$ is a weak solution, we have $\mathscr{E}_{x}=\mathscr{E}_{x} M=\mathfrak{D}_{x}$. Therefore, there exists a lattice gauge transformation $P=I+P_{1} t_{x}+P_{2} t_{x}^{2}+\cdots$ which sends the basis $\left(\sigma^{\prime}\right)$ into its $\Upsilon$-basis $\left(\varepsilon^{\prime}\right)$, where $\Upsilon$ is the Birkhoff form at $x$. The lattice $M^{\prime}$ spanned by $t_{x}^{D-T}\left(\sigma^{\prime}\right)$ is then necessarily logarithmic, according to Lemma 15 . We can effectively determine $M^{\prime}$ by truncating the gauge $P$ at order $d\left(M^{\prime}, D\right)-1=k_{n}-k_{1}-2$, and then applying Gantmacher's classical recursive formulæ (18). Then, the permutation lemma yields a monopole gauge transform $\Pi$ at $x$ so that the resulting trivialization $\overline{\mathscr{F}}$ is both $\mathrm{BG}$ and logarithmic. In this last basis, the connection has again the form (21), where the spectrum of the residue at $x$ gives the type of the modified logarithmic bundle $\mathscr{E} M$.

Proposition 56 implies that iterated applications of this procedure describe the set of all weak solutions to the Riemann-Hilbert problem. The strong problem is solvable if and only if one of the bundles $\overline{\mathscr{F}}$ has a 0 residue at $x$ in the orbit under these transformations.

5.5.3. The general case. For an arbitrary weak solution $\mathscr{E}$, we must start with the Deligne bundle $\mathfrak{D}$, for we only have the complete description of the local logarithmic lattices from the Deligne lattice. According to the description given in Proposition 52, any logarithmic lattice $N_{s} \in \boldsymbol{\Lambda}_{s}$ is given by an admissible pair $(\boldsymbol{F}, T)$ where $\boldsymbol{F}$ is a $\operatorname{Res}_{s}^{\mathfrak{D}} \nabla$-stable flag. If we put ourselves in the situation of Section 5.5.2, and consider a logarithmic BG trivialization $\mathscr{F}$ of $\mathfrak{D}$ at $x$, and identify $Y=\Gamma(X, \mathscr{F})$ to $\mathbb{C}^{n}$ by means of the basis $(\sigma)$, then the flag $\boldsymbol{F}$ can be viewed as a flag in $\mathbb{C}^{n}$ stable under the matrix $\widetilde{L}_{s}$. As exposed in Theorem 65 , the bundle $\mathscr{E}$ is then described by an element $\left(\boldsymbol{F}^{s}, D_{s}\right)_{s \in \mathscr{S}} \in \mathrm{RH}_{\chi}$ such that $\boldsymbol{F}^{s} \in \mathfrak{F l}_{\psi_{s}}(Y)$ and $D_{s} \in \mathbb{Z}^{n}\left(\boldsymbol{F}^{s}\right)$. In order to actually construct the lattice $N_{s}$, one should in principle reach first a Birkhoff form $\Upsilon_{z}$ in $\Lambda=\mathfrak{D}_{s}$. Since the Deligne lattice is nonresonant, it is possible to do so by a lattice gauge $P_{s}$ tangent to the identity, as described in Section 4.1.1. We know from Definition 14 that if we put $d_{s}=\Delta D_{s}-1$, the local gauge $P_{s}$ can be truncated to $t_{s}$-degree $d_{s}$, as remarked in the proof of Proposition 82. Assume for simplicity that $x=\infty \notin \mathscr{Y}$. Taking $Q_{s} \in \mathrm{GL}_{n}(\mathbb{C})$ that brings $(\sigma)$ to a basis respecting $\boldsymbol{F}^{s}$, the local gauge can be written as

$$
P_{s}=Q_{s}\left(I+\cdots+P_{d_{s}}^{(s)}(z-s)^{d_{s}}\right) .
$$

Take a rational interpolation $Z \in \mathrm{GL}_{n}(\mathbb{C}(z))$ of the local gauges $P_{s}$ to the prescribed orders $d_{s}$, and having only a singularity outside $\mathscr{Y}$ at $x$ (see, e.g., [van 
Barel et al. 1994]). The global basis of sections $(\tilde{\sigma})$ whose matrix in $(\sigma)$ is $P$ spans by construction a trivialization $\widetilde{\mathscr{F}}$ of $\mathscr{E}$ at $x$. Everything can be seen at $x$ as explained in the following scheme. Putting $\Lambda=\mathfrak{D}_{x}=\mathscr{E}_{x}$, we have

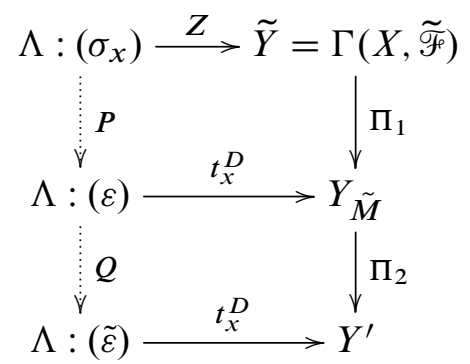

The effect of having changed the stalks over $\mathscr{Y}$ is translated in a purely local fashion by a change in the set $\Lambda^{0}$. Indeed, when $\Lambda$ represented the class of $[\mathfrak{D}]_{x}$, the germ $\left(\sigma_{x}\right)$ was a diagonal shift of a global basis. In this scheme however, $\Lambda$ represents the class $[\mathscr{E}]_{x}$, and we have now to apply the gauge $Z$, considered as an element of $\mathrm{GL}_{n}\left(\mathbb{C}\left(\left(t_{x}\right)\right)\right)$, to get a basis of global sections of $\tilde{Y}=\Gamma(X, \widetilde{\mathscr{F}})$. The monopole $\Pi_{1}$ corresponds to the construction of a BG trivialization, as in Section 3.2.1. The second gauge $\Pi_{2}$, which can be constructed by the enhanced permutation lemma (Proposition 38), brings the system to an optional logarithmic BG trivialization, where the system is again Fuchsian.

\section{References}

[Abramenko and Brown 2008] P. Abramenko and K. S. Brown, Buildings: Theory and applications, Graduate Texts in Mathematics 248, Springer, New York, 2008. MR 2009g:20055 Zbl 1214.20033

[André and Baldassarri 2001] Y. André and F. Baldassarri, De Rham cohomology of differential modules on algebraic varieties, Progress in Mathematics 189, Birkhäuser, Basel, 2001. MR 2002h:14031 Zbl 0995.14003

[Anosov and Bolibrukh 1994] D. V. Anosov and A. A. Bolibruch, The Riemann-Hilbert problem, Aspects of Mathematics 22, Friedr. Vieweg \& Sohn, Braunschweig, 1994. MR 95d:32024 Zbl 0801.34002

[Babbitt and Varadarajan 1983] D. G. Babbitt and V. S. Varadarajan, "Formal reduction theory of meromorphic differential equations: A group theoretic view", Pacific J. Math. 109:1 (1983), 1-80. MR 86b:34010 Zbl 0533.34010

[van Barel et al. 1994] M. van Barel, B. Beckermann, A. Bultheel, and G. Labahn, "Matrix rational interpolation with poles as interpolation points", pp. 137-148 in Nonlinear numerical methods and rational approximation, II: Proceedings of the 3rd International Conference (University of Antwerp, Belgium, 5-11 September 1993), edited by A. Cuyt, Mathematics and its Applications 296, Kluwer Academic Publishers, Dordrecht, 1994. Zbl 0803.41006

[Boalch 2011] P. P. Boalch, "Riemann-Hilbert for tame complex parahoric connections", Transform. Groups 16:1 (2011), 27-50. MR 2012m:14020 Zbl 1232.34117 
[Bolibrukh 1990] A. A. Bolibrukh, "The Riemann-Hilbert problem", Uspekhi Mat. Nauk 45:2(272) (1990), 3-47. In Russian; translated in Russ. Math. Surv. 45:2 (1990), 1-58. MR 92j:14014 Zbl 0706.34005

[Bolibrukh et al. 2006] A. A. Bolibruch, S. Malek, and C. Mitschi, "On the generalized RiemannHilbert problem with irregular singularities”, Expo. Math. 24:3 (2006), 235-272. MR 2007i:34148 Zbl 1106.34061

[Corel 2004] E. Corel, "On Fuchs' relation for linear differential systems", Compos. Math. 140:5 (2004), 1367-1398. MR 2005e:34270 Zbl 1070.34120

[Crawley-Boevey 2003] W. Crawley-Boevey, "On matrices in prescribed conjugacy classes with no common invariant subspace and sum zero", Duke Math. J. 118:2 (2003), 339-352. MR 2004g:16014 Zbl 1046.15013

[Deligne 1970] P. Deligne, Équations différentielles à points singuliers réguliers, Lecture Notes in Mathematics 163, Springer, Berlin, 1970. MR 54 \#5232 Zbl 0244.14004

[Gantmacher 1959] F. R. Gantmacher, The theory of matrices, II, Chelsea, New York, 1959. MR 21 \#6372c Zbl 0927.15002

[Garrett 1997] P. Garrett, Buildings and classical groups, Chapman-Hall, London, 1997. MR 98k: 20081 Zbl 0933.20019

[Gérard and Levelt 1973] R. Gérard and A. H. M. Levelt, "Invariants mesurant l'irrégularité en un point singulier des systèmes d'équations différentielles linéaires", Ann. Inst. Fourier (Grenoble) 23:1 (1973), 157-195. MR 49 \#10947 Zbl 0243.35016

[Ilyashenko and Yakovenko 2007] Y. Ilyashenko and S. Yakovenko, Lectures on analytic differential equations, Graduate Studies in Mathematics 86, American Mathematical Society, Providence, RI, 2007. MR 2009b:34001 Zbl 1186.34001

[Kostov 1992] V. P. Kostov, "Fuchsian linear systems on $\mathbf{C P}^{1}$ and the Riemann-Hilbert problem", $C$. R. Acad. Sci. Paris Sér. I Math. 315:2 (1992), 143-148. MR 94a:34007 Zbl 0772.34007

[Krause 1996] M. Krause, "A simple proof of the Gale-Ryser theorem”, Amer. Math. Monthly 103:4 (1996), 335-337. MR 1383671 Zbl 0855.05032

[Levelt 1961] A. H. M. Levelt, "Hypergeometric functions, II", Nederl. Akad. Wetensch. Proc. Ser. A 64 = Indag. Math. 23 (1961), 373-385. MR 25 \#1302 Zbl 0124.03602

[Malgrange 1996] B. Malgrange, "Connexions méromorphes, II: Le réseau canonique", Invent. Math. 124:1-3 (1996), 367-387. MR 97h:32060 Zbl 0849.32003

[Plemelj 1964] J. Plemelj, Problems in the sense of Riemann and Klein, Interscience Tracts in Pure and Applied Mathematics 16, Wiley, New York, 1964. MR 30 \#5008 Zbl 0124.28203

[Ronan 1989] M. Ronan, Lectures on buildings, Perspectives in Mathematics 7, Academic Press, Boston, MA, 1989. MR 90j:20001 Zbl 0694.51001

[Sabbah 2002] C. Sabbah, Déformations isomonodromiques et variétés de Frobenius, EDP Sciences, Paris, 2002. MR 2003m:32013 Zbl 1101.14001

[Treibich Kohn 1983] A. Treibich Kohn, "Un résultat de Plemelj”, pp. 307-312 in Mathematics and physics (Paris, 1979/1982), edited by L. Boutet de Monvel et al., Progr. Math. 37, Birkhäuser, Boston, 1983. MR 87c:32016 Zbl 0527.34007

Received December 3, 2010. Revised March 14, 2013. 
Université Des SCIENCES ET TeChNOLOGIES - LiLle 1

Cité SCIENTIFIQUe

59655 Villeneuve d'AsCQ

FRANCE

elie.compoint@math.univ-lille1.fr

EDUARDO COREL

UNIVERSITÉ D'EVRY-VAL-D'ESSONNE

IBGBI, 23 BOULEVARD DE FRANCE

91037 EVRY

FRANCE

eduardo.corel@genopole.cnrs.fr 


\title{
PACIFIC JOURNAL OF MATHEMATICS
}

\author{
msp.org/pjm
}

Founded in 1951 by E. F. Beckenbach (1906-1982) and F. Wolf (1904-1989)

\section{EDITORS}

V. S. Varadarajan (Managing Editor)

Department of Mathematics

University of California

Los Angeles, CA 90095-1555

pacific@math.ucla.edu

Paul Balmer

Department of Mathematics

University of California

Los Angeles, CA 90095-1555

balmer@math.ucla.edu

Daryl Cooper

Department of Mathematics

University of California

Santa Barbara, CA 93106-3080 cooper@math.ucsb.edu

Jiang-Hua $\mathrm{Lu}$

Department of Mathematics

The University of Hong Kong

Pokfulam Rd., Hong Kong jhlu@maths.hku.hk
Don Blasius

Department of Mathematics University of California

Los Angeles, CA 90095-1555

blasius@math.ucla.edu

Robert Finn

Department of Mathematics Stanford University

Stanford, CA 94305-2125

finn@math.stanford.edu

Sorin Popa

Department of Mathematics

University of California

Los Angeles, CA 90095-1555

popa@math.ucla.edu

Paul Yang

Department of Mathematics

Princeton University

Princeton NJ 08544-1000

yang@math.princeton.edu

\section{PRODUCTION}

Silvio Levy, Scientific Editor, production@msp.org

\section{SUPPORTING INSTITUTIONS}

ACADEMIA SINICA, TAIPEI

CALIFORNIA INST. OF TECHNOLOGY

INST. DE MATEMÁTICA PURA E APLICADA

KEIO UNIVERSITY

MATH. SCIENCES RESEARCH INSTITUTE

NEW MEXICO STATE UNIV.

OREGON STATE UNIV.

\author{
STANFORD UNIVERSITY \\ UNIV. OF BRITISH COLUMBIA \\ UNIV. OF CALIFORNIA, BERKELEY \\ UNIV. OF CALIFORNIA, DAVIS \\ UNIV. OF CALIFORNIA, LOS ANGELES \\ UNIV. OF CALIFORNIA, RIVERSIDE \\ UNIV. OF CALIFORNIA, SAN DIEGO \\ UNIV. OF CALIF., SANTA BARBARA
}

\author{
Vyjayanthi Chari \\ Department of Mathematics \\ University of California \\ Riverside, CA 92521-0135 \\ chari@math.ucr.edu \\ Kefeng Liu \\ Department of Mathematics \\ University of California \\ Los Angeles, CA 90095-1555 \\ liu@math.ucla.edu \\ Jie Qing \\ Department of Mathematics \\ University of California \\ Santa Cruz, CA 95064 \\ qing@cats.ucsc.edu
}

These supporting institutions contribute to the cost of publication of this Journal, but they are not owners or publishers and have no responsibility for its contents or policies.

See inside back cover or msp.org/pjm for submission instructions.

The subscription price for 2013 is US \$400/year for the electronic version, and \$485/year for print and electronic.

Subscriptions, requests for back issues and changes of subscribers address should be sent to Pacific Journal of Mathematics, P.O. Box 4163, Berkeley, CA 94704-0163, U.S.A. The Pacific Journal of Mathematics is indexed by Mathematical Reviews, Zentralblatt MATH, PASCAL CNRS Index, Referativnyi Zhurnal, Current Mathematical Publications and the Science Citation Index.

The Pacific Journal of Mathematics (ISSN 0030-8730) at the University of California, c/o Department of Mathematics, 798 Evans Hall \#3840, Berkeley, CA 94720-3840, is published monthly except July and August. Periodical rate postage paid at Berkeley, CA 94704, and additional mailing offices. POSTMASTER: send address changes to Pacific Journal of Mathematics, P.O. Box 4163, Berkeley, CA 94704-0163.

PJM peer review and production are managed by EditFLOW ${ }^{\circledR}$ from Mathematical Sciences Publishers.

PUBLISHED BY

mathematical sciences publishers

nonprofit scientific publishing

http://msp.org/

(C) 2013 Mathematical Sciences Publishers 


\section{PACIFIC JOURNAL OF MATHEMATICS}

Volume $263 \quad$ No. $2 \quad$ June 2013

Realizations of $B C_{r}$-graded intersection matrix algebras with grading 257 subalgebras of type $B_{r}, r \geq 3$

SANDEEP BHARGAVA and YUN GAO

Stable flags, trivializations and regular connections

283

Elie COMPOINT and EDUARDo COREL

Elliptic aliquot cycles of fixed length

353

NATHAN JONES

Asymptotic $L^{4}$ norm of polynomials derived from characters

DANIEL J. KATZ

Degree-three spin Hurwitz numbers

JUNHO LEE

$\left(\mathbb{Z}_{2}\right)^{3}$-colorings and right-angled hyperbolic 3-manifolds

YOULIN LI and JiMING MA

Real closed separation theorems and applications to group algebras

Tim NETZER and ANDREAS THOM

Uniqueness theorem for ordinary differential equations with Hölder continuity

YifEI PAN, MEI WANG and Yu YAN

An analogue to the Witt identity

G. A. T. F. DA CostA and G. A. ZimmermanN

On the classification of stable solutions to biharmonic problems in large dimensions

Juncheng Wei, Xingwang Xu and Wen Yang 